Lehrstuhl für Hochfrequenztechnik

der Technischen Universität München

\title{
Network Methods Applied to Multilayered Cylindrical Radiating Structures.
}

\section{Bruno Biscontini}

Vollständiger Abdruck der von der Fakultät für Elektrotechnik und Informationstechnik der Technischen Universität München zur Erlangung des akademischen Grades eines

\section{Doktor-Ingenieurs}

genehmigten Dissertation.

Vorsitzender: $\quad$ Univ.-Prof. P. Lugli, Ph.D.

Prüfer der Dissertation: 1. Univ.-Prof. Dr. techn. P. Russer

2. Prof. R. Sorrentino;

Univ. di Perugia/Italien

Die Dissertation wurde am 21.12.2005 bei der Technischen Universität München eingereicht und durch die Fakultät für Elektrotechnik und Informationstechnik am 11.07.2006 angenommen. 


\section{Abstract}

This work deals with the development of methods, algorithms and software implementations, for the analysis, design and optimization of multilayered radiating cylindrical structures.

A method based on the integral equation method (IEM) in connection with method of moment (MOM) is developed. Dealing with IEM a key problem is the computation of the Green's function.

A novel method to compute the Green's function for multilayered cylindrical structures is presented. Making use of the symmetry properties of the cylindrical structure a circuit description of multilayered cylindrical structure in spectral domain (SD) is developed. The circuit model is based on generalized transmission lines (GTL). The GTL method is used to compute the spectral domain dyadic Green's function components. The space domain Green's functions are computed using a quasi-analytical approach. This means that we approximate the spectral domain Green's functions using a poles/residues expansion into a series of exponential functions. The poles and residues are estimated using the generalized pencil of function (GPOF) method. Having the spectral domain components of the dyadic Green's function represented by exponential functions, the space domain Green's function is performed analytically.

The convergence of the cylindrical Green's function near the source region are treated in details. For this purpose we consider that the Green's function is given in terms a series of cylindrical waves functions. Due to the singularity of the Green's function in the origin, the correct and fast convergence in the near field is an important issue. In this work we present a convergence analysis of the cylindrical Green's function. In this context the availability of simple and accurate reference models for the evaluation of the method in the near field region is a key point. From the analytical point of view, the problem is solved by the theory of distribution. In this 
work, we discuss the limitations of the classical description of the source region given by the theory of distribution from the numerical implementation point of view.

In order to show the potentialities and to validate the method, we present several challenge applications. The Green's function is used with MOM for the modelling of conformal cylindrical antennas embedded in a cylindrical radome structure and circular conformal antenna array mounded around a cylindrical mast acting as reflector. In order to demonstrate one important advantage rotational symmetric structures, we present beamforming and azimuthal scanning algorithms combined with electromagnetic simulations of the antenna array without and with a three layers radome. Although beam-shaping has advanced properties, the proposed method for numerical modelling is rather fast and efficient. Indeed, due to the fast semianalytical approach presented in this work, we show that the computation can be carried out quasi simultaneously. The results are compared with measurements and with results obtained with others commercial CADs. 


\section{Acknowledgments}

First of all I am indebted to Professor Peter Russer for the opportunity he offered me to undertake this experience and for his guidance. It is a pleasure to acknowledge the contributions to my understanding of this subject by a number of colleagues. My deep gratitude goes furthermore to a number of persons who have shared with me these past years in the good as well as bad times enriching my professional and private life. Among them my wife and my son for their patience, of course not least, my parents without whom all this would not be possible.

My gratitude goes also to Mr. Franz Demmel for the many interesting and fruitful discussions and to Rohde\& Schwarz for the support to this work.

Munich July 15st 2005. 



\section{Preface}

In this manuscript exterior calculus formalism is adopted. A short review of the relation with the classical vector representation is given in the Appendix E. A more complete treatment of the topic can be found in $[21,29,55]$. It is interesting that this modern point of view was anticipated by Maxwell himself:

...We are here led to considerations belonging to the Geometry of Position, a subject which, though its importance was pointed out by Leibnitz and illustrated by Gauss, has been little studied. [51]. 



\section{Contents}

1. Abstract iii

2. Acknowledgments v

3. Preface vii

4. Introduction 1

4.1. Problem Formulation . . . . . . . . . . . . . . . . . . . . 1

4.2. Motivations . . . . . . . . . . . . . . . 1

4.3. Comparison with the State of the Art . . . . . . . 3

4.4. Structure of the Manuscript . . . . . . . . . . . . 5

5. Green's function definition 7

5.1. Mathematical Preliminaries . . . . . . . . . . . . 7

5.2. Field representation in terms of potentials . . . . . . . . 12

6. Green's function for multilayered cylindrical radiating structures $\quad 17$

6.1. Dyadic Green's function definition for multilayered cylindri-

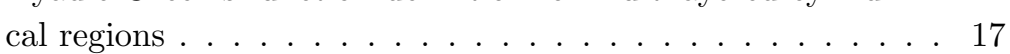

6.2. Network description . . . . . . . . . . . . . . 30

6.3. Space Domain Green's functions . . . . . . . . . . . . . . 34

6.4. The Generalized Pencil of Function(GPOF) Algorithm . . . . 43

7. Validation of the GPOF and Space Domain Green's Functions 49

7.1. GPOF Validation . . . . . . . . . . . . . . . . . 49 49 
7.2. Numerical Results for the GPOF . . . . . . . . . . . . . 53

7.3. Nearfield Green's Function . . . . . . . . . . . . . . 62

8. Application and Validation $\quad 85$

8.1. Moment Method Formulation . . . . . . . . . . . . . 85

8.2. Application and Validation . . . . . . . . . . . 87

8.3. Comparison of Time/Memory Performance . . . . . . . . . 9 93

8.4. Beamforming and Azimuth Scanning Application . . . . . . . 98

9. Conclusions and Discussions $\quad 101$

9.1. Outlook ...................... 102

A. Appendix $\quad 105$

A.1. Potential Ansatz for $z$ and $\varphi$-oriented current . . . . . . . 105

B. Appendix $\quad 107$

B.1. Complex Path Integration . . . . . . . . . . . . 107

C. Appendix 109

C.1. A static recursive approach . . . . . . . . . . . . . 109

D. Appendix 113

D.1. Field Computations . . . . . . . . . . . . 113

E. Appendix $\quad \mathbf{1 1 9}$

E.1. Exterior Differential forms . . . . . . . . . . . . . . 119 


\section{Introduction}

\subsection{Problem Formulation}

This work deals with the development of theoretical methods, algorithms, software and hardware implementations for the analysis, design and optimization of multilayered radiating cylindrical structures.

\subsection{Motivations}

Due to their excellent radiation characteristics and beam forming performance, multilayered cylindrical antennas are growing of importance in a multitude of RF communications and radionavigation applications. Over the years applications spam direction finding, radar, sonar and smart antenna [43].

Even though the symmetrical properties of the structure simplify the modeling, the analysis and design of multilayered cylindrical radiating structure is a real challenge. The difficulties, for standard volume discretaizing methods, arise from the complexity of the electromagnetic problem definition itself. Indeed, the fine structure details (multiple thin layers), the presence of different dielectric materials, a high aspect ratio, cylindrical shape, unbounded radiating problem, require a fine meshing and excessive time and memory resources consumption are needed.

As an example, we have simulated, using WinFEKO [56], a hollow dielectric cylinder of relative permittivity $\varepsilon=4.5$ [8]. A short electric dipole in axial direction, resonating at $800 \mathrm{MHz}$, was embedded inside the dielectric cylinder, Figure 1. For a normal discretization step according to the WinFEKO criterium [56]), we have used 500 MBytes fast memory and the simulation time was around $20 \mathrm{~min}$ at one frequency. It follows that 


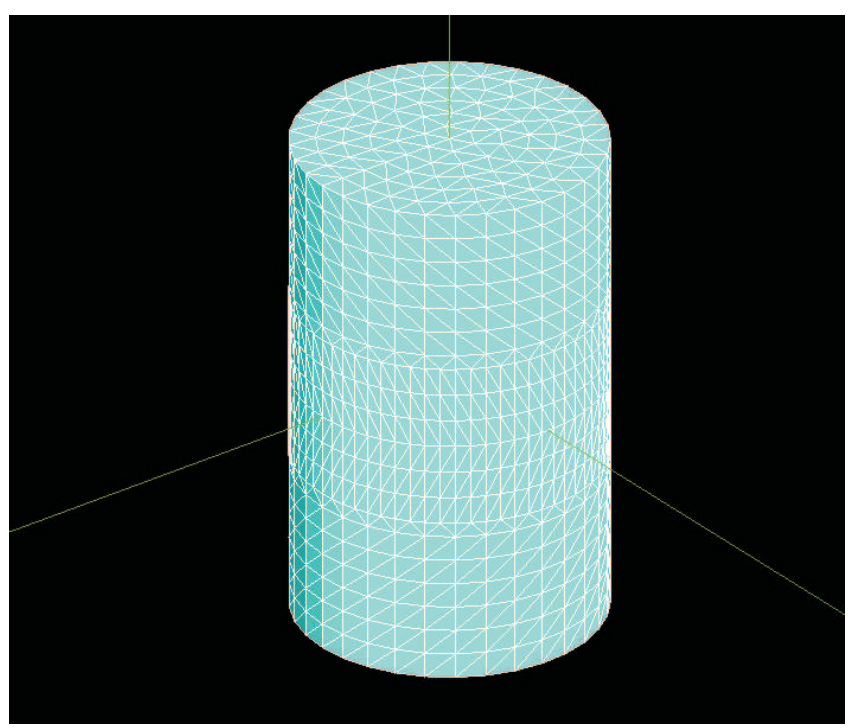

Figure 1.: Structure modelled with WinFEKO.

for more complicated problems and higher frequencies, analysis, design and optimization of cylindrical multilayered conformal antennas, radome etc., can be a complex engineering task. More examples using other commercial CAD tools will be given in Chapter 8 .

An alternative solution of the problem can be a more sophisticated simulation environment like parallel processing in Grid environment $[9,10,11]$. However, such an emerging approach, would not offer the possibility of a systematic way to investigate and design such cylindrical structures. Conversely, in such problems where significant geometry symmetry properties can be exploited, a more understanding view of the electromagnetic problem can be used to introduce fast and accurate semianalytical simulation techniques. In this work an alternative to the standard integral equation method (IEM) in connection with the method of moments (MOM) [12,13,8] is presented. 


\subsection{Comparison with the State of the Art}

Green functions for cylindrical structures have been treated in numerous publications $[2,3,7,4]$. Following the field solution type, and the usual convention terminology used in the literature, two basic approach can be followed to compute the Green's function: the mixed potential integral equation (MPIE) and electric field integral equation (EFIE). Following the definition of the field potential, the MPIE exhibits a better numerical behavior of the EFIE in the source region since the singularity is one order lower [49,41]. In [1] analytical closed form expressions are given for twoand three-layered cylindrical structures. The approach, basically follows the modal expansion representation given in [7]. Also a recursive algorithm formulation to compute the spectral domain Green's function has been reported in [6] which is basically a cylindrical wave expansion of the mixed potential integral equation (MPIE). In this case, the space domain components of the dyadic Green's function are computed using a poles/residues expansion approximation for the spectral domain Green's functions introduced in $[35,17]$. Here the spectral domain Green's function is represented into series of cylindrical eigenmodes estimated via the generalized pencil of function method (GPOF) which is basically a series of exponential functions [17]. Therefore, after the approximation, the inverse Fourier integral over the complex plane $k_{z}$ could be computed analytically in easy and fast way [6]. Historically, this method is usually referred as image technique and was already used in 1988 [45] and for planar structures in [35,46] in 1991 and 1992 respectively. One of the key problem of this method is the slow convergence of the expansion into series of cylindrical waves of the Green's function. In particular if $\left(\rho^{\prime}, \varphi^{\prime}, z^{\prime}\right)$ are the source coordinates, for $\rho^{\prime}<<\rho$, ( $\rho$ is the observation point), the cylindrical waves expansion forms a fast convergent series. This is the case of [6] and [48]. Conversely, if the condition $\rho^{\prime}<<\rho$ is not satisfied, i.e., $\rho^{\prime}$ and $\rho$ are near each other, the cylindrical wave expansion yields a slowly convergent series [47]. The convergence can be improved by extracting the slowly convergent part of the Green's function as reported in [47]. In the latter the convergence was investigated for a given $\rho^{\prime}$ and $\rho$ and moving over $z$. The obtained Green's functions in [47] have been then compared with [6] chosen as reference model. 
The first contribution of this work is to introduce a network description in spectral domain of the multilayered cylindrical structure. The network analogy is given introducing a generalized transmission line (GTL) model for the cylindrical layers in spectral domain [13,12]. Such an approach has several advantages. First in the implementation stage of the Green's function the electromagnetic boundary conditions are described simply via connection with the ports and therefore the addition of the cylindrical layer implies only an addition of a GTL. Therefore adding and removing layers is a relatively easy task. Also relevant numerical advantages arise by the introduction of the GTL description. Indeed, having a GTL model, we can use e.g., a chain of ABCD matrixes which in lossless case and reciprocal media, has the property to have a unitary determinant [52]. As a consequence, important numerical problems arising from matrixes inversion can be easily handled. Also, the GTL introduces an additional important insight in the design and optimization stage $[12,8,14]$.

To compute the space domain components of the Greens's function we have used a GPOF [6] approximation in spectral domain and therefore the inverse Fourier integral could be analytically computed.

The second contribute of this work is a three dimensional convergence analysis in the source region introducing accurate reference models. As mentioned before one of the key problems is the analysis of the Green's function convergence for small $\left|\mathbf{r}-\mathbf{r}^{\prime}\right|$ where $\mathbf{r}^{\prime}$ describes the source region and $\mathbf{r}$ the observation space. In this work we have performed a three dimensional convergence analysis of the Green's function and shown the convergence properties moving along $\rho, \varphi, z$ in the near field region. Also accurate reference models for near field computation have been discussed and introduced. The reference model is based on static field approximation for small space variation $\left|\mathbf{r}-\mathbf{r}^{\prime}\right|$ and method of moment (MOM).

\subsubsection{Applications of the GTL-Green's function and Validation}

The obtained Green's function is used to model via MOM a conformal to cylindrical surface axial dipole and circular array of conformal dipoles, confined into a multilayered cylindrical structures. The dipole/s are mounted 
around a cylindrical reflector. To show the power of the method, simple beamforming and azimuth scanning algorithm are performed on the fly together with the electromagnetic simulation and the performance is shown in term of computational time. The results are validated with other commercial CAD and measurements.

\subsection{Structure of the Manuscript}

In Chapter 5 the necessary definitions and mathematical preliminaries are given. In Chapter 6 the GTL model of the cylindrical structure in spectral domain and the Green's function computations are presented. In Chapter 7 the validation and convergence of the Green's function in the near field are presented. The Chapter 8 deals with the use of the Green's function in MOM application of measurements. In Appendixes D.1.1 some particular potential Ansatz useful for implementations purposes are given. In Appendix B some used mathematical tools used in the theoretical development are shortly reviewed. In Appendix $\mathrm{C}$ implementation issues are discussed. In the Appendix $\mathrm{D}$ some useful asymptotic approximation useful for the far-field pattern visualization are reported. Finally in Appendix E a short review of the differential form calculus is given. 



\section{Green's function definition}

\subsection{Mathematical Preliminaries}

In general, from the linearity of the Maxwell's equations, the field can be expressed in terms of the excitation currents by the integral representations as follows

$$
\mathcal{E}(\mathbf{r})=j \omega \mu \int_{V^{\prime}} \mathcal{G}_{e}\left(\mathbf{r}, \mathbf{r}^{\prime}\right) \wedge \mathcal{J}\left(\mathbf{r}^{\prime}\right)
$$

where $V^{\prime}$ is the volume in which the sources are non-vanishing, $\mathbf{r}$ is the observation point, $\mathbf{r}^{\prime}$ is the location of the source. The definition of the Green's function is given considering three dimensional current distributions corresponding to three infinitesimal electric dipoles at $\mathbf{r}=\mathbf{r}^{\prime}$ and oriented in three-orthogonal directions and having a unitary Dirac distribution of the current. Since any arbitrary system of curvilinear system of coordinates with coordinates $u, v, w$ can be defined via embedding in the three-dimensional Cartesian coordinate system $x y, z$ as in [21], we start using Cartesian coordinates. In this case, the above mentioned Dirac current distribution can be written as follows:

$$
\mathcal{J}(\mathbf{r})=J_{0_{x}} \delta\left(\mathbf{r}-\mathbf{r}^{\prime}\right) \mathrm{d} y \wedge \mathrm{d} z+J_{0_{y}} \delta\left(\mathbf{r}-\mathbf{r}^{\prime}\right) \mathrm{d} z \wedge \mathrm{d} x+J_{0_{z}} \delta\left(\mathbf{r}-\mathbf{r}^{\prime}\right) \mathrm{d} x \wedge \mathrm{d} y
$$

To compute the field at point $\mathbf{r}$ excited by the source defined in (2) and located at point $\mathbf{r}^{\prime}$ we use the Green's double one form [21,30,29] defined as follows:

$$
\begin{aligned}
\mathcal{G}_{s}\left(\mathbf{r}, \mathbf{r}^{\prime}\right)= & G_{x x^{\prime}} \mathrm{d} x \mathrm{~d} x^{\prime}+G_{x y^{\prime}} \mathrm{d} x \mathrm{~d} y^{\prime}+G_{x z^{\prime}} \mathrm{d} x \mathrm{~d} z^{\prime} \\
& +G_{y x} \mathrm{~d} y \mathrm{~d} x^{\prime}+G_{y y^{\prime}} \mathrm{d} y \mathrm{~d} y^{\prime}+G_{y z^{\prime}} \mathrm{d} y \mathrm{~d} z^{\prime} \\
& +G_{z x^{\prime}} \mathrm{d} z \mathrm{~d} x^{\prime}+G_{z y^{\prime}} \mathrm{d} z \mathrm{~d} y^{\prime}+G_{z z^{\prime}} \mathrm{d} z \mathrm{~d} z^{\prime}
\end{aligned}
$$

The Green's double form relates the observation space $\mathbf{r}$ to the source space $\mathbf{r}^{\prime}$. We introduce a curvilinear space of coordinates $u, v, w$. We assume it to 
be an orthogonal coordinate system. In this case the curvilinear system of coordinates can be directly defined from the Cartesian system of coordinates calculating the metric coefficients [21]. In this case (3) can be directly extend as follows:

$$
\begin{array}{r}
\mathcal{G}_{e}\left(\mathbf{r}, \mathbf{r}^{\prime}\right)=G_{u u^{\prime}} g_{1} g_{1}^{\prime} \mathrm{d} u \mathrm{~d} u^{\prime}+g_{1} g_{2}^{\prime} G_{u v^{\prime}} \mathrm{d} u \mathrm{~d} v^{\prime}+g_{1} g_{3}^{\prime} G_{u w^{\prime}} \mathrm{d} u \mathrm{~d} w^{\prime} \\
+g_{2} g_{1}^{\prime} G_{v u} \mathrm{~d} v \mathrm{~d} u^{\prime}+g_{2} g_{2}^{\prime} G_{v v^{\prime}} \mathrm{d} v \mathrm{~d} v^{\prime}+g_{2} g_{3}^{\prime} G_{v w^{\prime}} \mathrm{d} v \mathrm{~d} w^{\prime} \\
+g_{3} g_{1}^{\prime} G_{w u^{\prime}} \mathrm{d} w \mathrm{~d} u^{\prime}+g_{3} g_{2}^{\prime} G_{w v^{\prime}} \mathrm{d} w \mathrm{~d} v^{\prime}+g_{3} g_{3}^{\prime} G_{w w^{\prime}} \mathrm{d} w \mathrm{~d} w^{\prime}
\end{array}
$$

where $g_{1}, g_{2}, g_{3}$ and $g_{1}^{\prime}, g_{2}^{\prime}, g_{3}^{\prime}$ are the metric coefficients of the observation and source space respectively. In the same way we can write (2) as follows

$$
\begin{array}{r}
\mathcal{J}(\mathbf{r})=J_{0_{u}} \delta\left(\mathbf{r}-\mathbf{r}^{\prime}\right) g_{2} g_{3} \mathrm{~d} w \wedge \mathrm{d} u+J_{0_{v}} \delta\left(\mathbf{r}-\mathbf{r}^{\prime}\right) g_{1} g_{3} \mathrm{~d} u \wedge \mathrm{d} w+ \\
J_{0_{w}} \delta\left(\mathbf{r}-\mathbf{r}^{\prime}\right) g_{1} g_{2} \mathrm{~d} u \wedge \mathrm{d} y
\end{array}
$$

We introduce now the unit one-forms:

$$
\begin{array}{ccc}
s_{1}=g_{1} \mathrm{~d} u & s_{2}=g_{2} \mathrm{~d} v & s_{3}=g_{3} \mathrm{~d} w \\
s_{1}^{\prime}=g_{1}^{\prime} \mathrm{d} u^{\prime} & s_{2}^{\prime}=g_{2}^{\prime} \mathrm{d} v^{\prime} & s_{3}^{\prime}=g_{3}^{\prime} \mathrm{d} w^{\prime}
\end{array}
$$

where the symbol ' indicates the source space. Using (6) in (5) and (4) and substituting in (199) we obtain

$$
\begin{aligned}
& \mathcal{E}(\mathbf{r})=\int_{V^{\prime}} \mathcal{G}_{e}\left(\mathbf{r}, \mathbf{r}^{\prime}\right) \wedge \delta\left(\mathbf{r}-\mathbf{r}^{\prime}\right) s_{2}^{\prime} \wedge s_{3}^{\prime}+\int_{V^{\prime}} \mathcal{G}_{e}\left(\mathbf{r}, \mathbf{r}^{\prime}\right) \wedge \delta\left(\mathbf{r}-\mathbf{r}^{\prime}\right) s_{1}^{\prime} \wedge s_{3}^{\prime}+ \\
& \int_{V^{\prime}} \mathcal{G}_{e}\left(\mathbf{r}, \mathbf{r}^{\prime}\right) \wedge \delta\left(\mathbf{r}-\mathbf{r}^{\prime}\right) s_{1}^{\prime} \wedge s_{2}^{\prime}
\end{aligned}
$$

Let us consider the first integral:

$$
\begin{gathered}
\left.\mathcal{E}(\mathbf{r})=\int_{V^{\prime}} \mathcal{G}_{e}\left(\mathbf{r}, \mathbf{r}^{\prime}\right) \wedge \delta\left(\mathbf{r}-\mathbf{r}^{\prime}\right)\right) s_{2}^{\prime} \wedge s_{3}^{\prime}= \\
\int_{V^{\prime}}\left[G_{u u^{\prime}}\left(\mathbf{r}, \mathbf{r}^{\prime}\right) s_{1}+G_{v u^{\prime}}\left(\mathbf{r}, \mathbf{r}^{\prime}\right) s_{2}+G_{w u^{\prime}}\left(\mathbf{r}, \mathbf{r}^{\prime}\right) s_{3}\right] \delta\left(\mathbf{r}-\mathbf{r}^{\prime}\right)\left(s_{1}^{\prime} \wedge s_{2}^{\prime} \wedge s_{3}^{\prime}\right)= \\
G_{u u^{\prime}}\left(\mathbf{r}, \mathbf{r}^{\prime}\right) s_{1}+G_{v u^{\prime}}\left(\mathbf{r}, \mathbf{r}^{\prime}\right) s_{2}+G_{w u^{\prime}}\left(\mathbf{r}, \mathbf{r}^{\prime}\right) s_{3}
\end{gathered}
$$

In the same manner we can treat all the integrals of (7). Defining the $\mathcal{E}(\mathbf{r})$ as one form in the following way

$$
\mathcal{E}(\mathbf{r})=E_{u} s_{1}+E_{v} s_{2}+E_{w} s_{3}
$$

and considering (8) and (7) we can conclude

$$
G_{p q}\left(\mathbf{r}, \mathbf{r}^{\prime}\right)=\left.E_{p}(\mathbf{r})\right|_{\mathcal{J}(\mathbf{r})=\delta\left(\mathbf{r}-\mathbf{r}^{\prime}\right) \mathbf{u}_{q}}
$$


where $G_{p q}\left(\mathbf{r}, \mathbf{r}^{\prime}\right)$ are the scalar components of the dyadic Green's function in direction $p$ due to a $q$-oriented Dirac source, $E_{p}$ are the three scalar orthogonal components of the electric field and $\mathbf{u}_{q}=s_{k} \wedge s_{h}$ for $k, h=1,2,3$ and $k \neq h$ are the orientation of the sources in the local system of coordinate. Therefore the electric dyadic Green's function is completely determined if the electric fields produced by the orthogonal Dirac dipoles placed at $\mathbf{r}^{\prime}$ are known.

\subsubsection{The Sturm-Liouville Differential Equation}

We assume an electromagnetic boundary value problem in which the solution can be given by separation of variables. If the boundary conditions for the problem are homogeneous, one of the ordinary differential equation will give a Sturm-Liouville type problem, which leads to a set of orthogonal eigenfunctions as solutions.

In the following we will discuss and review some important basic properties of the Sturm-Liouville differential equation.

We start from the one-dimensional scalar homogeneous Sturm-Liouville differential equation given in the following

$$
\frac{d}{d x}\left[p(x) \frac{d \psi(x)}{d x}\right]+[q(x)+\lambda \sigma(x)] \psi(x)=0,
$$

where $\psi(x)$ is the unknown solution function, $p(x), q(x)$ and $\sigma(x)$ are real and continuous within the definition range. Typically, $p(x)$ and $\sigma(x)$ are positive and $\lambda$ may be complex. If the interval of definition of the problem is $x \epsilon[a, b]$, then the solution of (11) are fixed if boundary conditions are specified. The common boundary conditions are given by the following

$$
\begin{array}{lll}
\psi+c_{a} \frac{d \psi}{d x}=0 & \text { for } & x=a, \\
\psi+c_{b} \frac{d \psi}{d x}=0 & \text { for } & x=b .
\end{array}
$$

where $c_{a}$ and $c_{b}$ are real. For the given boundary conditions, we express the solution as infinite series of eigenfunctions $\psi_{n}$ with the corresponding eigenvalue $\lambda_{n}[21,31]$. This is possible since $\psi$ form an orthogonal set of functions in the interval of definition in all the cases [31]. Therefore they form also a 
complete set of function which may be used to expand any arbitrary piecewise continuous function $\psi(x)$ into a Fourier-type series. Accordingly, we express the boundary value problem solution as

$$
\psi(x)=\sum_{n=1}^{\infty} a_{n} \psi_{n}(x)
$$

where $\psi(x)$ are the normalized basis functions by $\sigma(x)$ in the definition interval $x \epsilon[a, b]$ as follows

$$
\int_{a}^{b} \sigma(x) \psi_{n} \psi_{m} \mathrm{~d} x= \begin{cases}1, & n=m \\ 0, & n \neq m\end{cases}
$$

and the unknown coefficients $a_{n}$ can be obtained as in [21,31]

$$
a_{n}=\int_{a}^{b} \sigma \psi_{n}^{*} \psi(x) \mathrm{d} x
$$

We introduce a perturbation term $g(x)$. In this case the the inhomogeneous Sturm-Liouville differential equation for the given perturbation is of the following form

$$
\frac{d}{d x}\left[p(x) \frac{d \psi(x)}{d x}\right]+[q(x)+\lambda \sigma(x)] \psi(x)=g(x) .
$$

If the perturbation term is described by a Dirac distribution at point $x^{\prime}$ $\delta\left(x-x^{\prime}\right)$ then the solution of (16) is a Green's function $G\left(x, x^{\prime}\right)$ that satisfies the following inhomogeneous Sturm-Liouville differential equation

$$
\frac{d}{d x}\left[p(x) \frac{d G\left(x, x^{\prime}\right)}{d x}\right]+[q(x)+\lambda \sigma(x)] G\left(x, x^{\prime}\right)=\delta\left(x-x^{\prime}\right),
$$

whereas $G\left(x, x^{\prime}\right)$ must fulfill appropriate boundary conditions. We assume for the inhomogeneous problem the same boundary conditions as in the homogeneous case (12). Since (17) is linear, the solution can be expressed in terms of the perturbation term by the integral representations as follows

$$
\psi(x)=\int_{x^{\prime}=a}^{b} G\left(x, x^{\prime}\right) g\left(x^{\prime}\right) \mathrm{d} x^{\prime}
$$


Considering $g(x)=\delta\left(x-x^{\prime}\right)$ and using the sampling property of the Dirac function we obtain

$$
\psi(x)=G\left(x, x^{\prime}\right)
$$

where the Green's function $G\left(x, x^{\prime}\right)$ may be considered as function of the variable $x$ with parameter $x^{\prime}$ indicating the position of the perturbation. Considering that $\psi_{n}(x)$ are a complete set of basis function, according to (19) the Green's function can be expanded into eigenfunctions as in the homogeneous case as follows

$$
G\left(x, x^{\prime}\right)=\sum_{n=-\infty}^{\infty} a_{n}\left(x^{\prime}\right) \psi_{n}(x)
$$

In this case the complex amplitudes $a_{n}\left(x^{\prime}\right)$ of the basis functions are dependent on the location $x^{\prime}$ and in general magnitude of the perturbation term. There are several approaches for determining the unknown coefficients $a_{n}\left(x^{\prime}\right)[7,21,31,32]$ which bring to different representation of the Green's function. For completeness, in the following the spectral representation will be discussed while a more physical approach will be introduced and explained later. We first expand also the Dirac perturbation in eigenfunctions using the same basis functions $\psi_{n}(x)$ as follows:

$$
\delta\left(x-x^{\prime}\right)=\sum_{n=1}^{\infty} a_{n} \psi_{n}(x)
$$

Using (15) we compute the unknown coefficients $a_{n}$ as follows

$$
a_{n}=\int_{x=a}^{b} \sigma \psi_{n}^{*}(x) \psi(x) \mathrm{d} x=\int_{x=a}^{b} \sigma \psi_{n}^{*}(x) \delta\left(x-x^{\prime}\right) \mathrm{d} x=\sigma \psi_{n}^{*}\left(x^{\prime}\right)
$$

inserting the (22) into (21) we obtain

$$
\delta\left(x-x^{\prime}\right)=\sum_{n=1}^{\infty} \sigma \psi_{n}^{*}\left(x^{\prime}\right) \psi_{n}(x)
$$

Inserting (20) and (23) into (17) we obtain

$$
\sum_{n=1}^{\infty}\left\{\frac{d}{d x}\left[p(x) \frac{d}{d x}\right]+[q(x)+\lambda \sigma(x)]\right\} a_{n}\left(x^{\prime}\right) \psi_{n}(x)=\sum_{n=1}^{\infty} \sigma \psi_{n}^{*}\left(x^{\prime}\right) \psi_{n}(x)
$$


Since the eigenfunctions $\psi_{n}(n)$ must satisfy the eigenvalue equation (11) for $\lambda=\lambda_{n}$

$$
\left.\frac{d}{d x}\left[p(x) \frac{d \psi(x)}{d x}\right]+\left[q(x)+\lambda_{n} \sigma(x)\right] \psi_{(} x\right)=0,
$$

the $(20$ becomes

$$
\sum_{n=1}^{\infty}\left(\lambda-\lambda_{n}\right) a_{n}\left(x^{\prime}\right) \psi_{n}(x)=\sum_{n=1}^{\infty} \sigma \psi_{n}^{*}\left(x^{\prime}\right) \psi_{n}(x) .
$$

therefore we conclude that for each $n$

$$
a_{n}\left(x^{\prime}\right)=\frac{\psi_{n}^{*}\left(x^{\prime}\right)}{\lambda_{n}-\lambda} .
$$

Substituting the (27) into (20) we obtain the spectral representation of the Green's function $G\left(x, x^{\prime}\right)$

$$
G\left(x, x^{\prime}\right)=\sum_{n=-\infty}^{\infty} \frac{\psi_{n}^{*}\left(x^{\prime}\right) \psi_{n}(x)}{\lambda-\lambda_{n}}
$$

where we observe the symmetry of the Green's function in $x$ and $x^{\prime}$ and the poles of the Green's function at $\lambda=\lambda_{n}$

\subsection{Field representation in terms of potentials}

We consider time-harmonic fields and therefore we will consider time-harmonic potentials. In this case, an $e^{j \omega t}$ time-dependence is assumed and suppressed. In presence of electric sources and in homogeneous regions the Faraday's law and Ampère's law can be expressed in complex phasor representation as follows

$$
\begin{aligned}
\mathrm{d} \underline{\mathcal{H}} & =j \omega \underline{\varepsilon} \star \underline{\mathcal{E}}+\underline{\mathcal{J}} \\
\mathrm{d} \underline{\mathcal{E}} & =-j \omega \underline{\mu} \star \underline{\mathcal{H}}
\end{aligned}
$$

where $\underline{\varepsilon}$ and $\underline{\mu}$ are the complex permittivity and permeability respectively, $\underline{\mathcal{J}}$ is the impressed electric current phasor. The equation (29) provides a 
system of six scalar equation to be solved to obtain the scalar component of the electric and magnetic fields. Moreover the magnetic flux continuity and Gauss' law must also be considered

$$
\begin{aligned}
& \mathrm{d} \underline{\mathcal{B}}=0 \\
& \mathrm{~d} \underline{\mathcal{D}}=\underline{\mathcal{Q}}
\end{aligned}
$$

where $\underline{\mathcal{Q}}$ is electric charge density form. The (30a) and (30b) are related with the fields via constitutive relations as follows

$$
\begin{array}{ll}
\underline{\mathcal{B}}=\star \underline{\mu} \underline{\mathcal{H}} & \underline{\mathcal{H}}=\frac{1}{\underline{\mu}} \star \underline{\mathcal{B}} \\
\underline{\mathcal{D}}=\star \star \underline{\mathcal{E}} & \underline{\mathcal{E}}=\frac{1}{\underline{\varepsilon}} \star \underline{\mathcal{D}}
\end{array}
$$

The (29) and (30) constitute a system of 12 scalar equations which can be generally simplified introducing some potential functions in terms of which the fields can be expressed. According to (30a) and (31b), $\mathrm{d} \star \underline{\mathcal{H}}=0$ the magnetic field is divergence-free. Using the Poincaré's lemma [21], $\underline{\mathcal{H}}$ can be expressed as the exterior derivative of a one form $\mathcal{A}$ as follows

$$
\underline{\mathcal{H}}=\frac{1}{\underline{\mu}} \star \mathrm{d} \mathcal{A}
$$

where the $\mathcal{A}$ is called magnetic vector potential form and any two-form $\star \underline{\mathcal{H}}$ with varnishing exterior derivative can be expressed as exterior derivative of one-form [21]. Such a two form describes a solenoidal field. In the vector potential form $\mathcal{A}$ is not divergence-free since i.e. $\mathrm{d} \star \mathcal{A} \neq 0$ and therefore has a lamellar part and has also a solenoidal part since in general for oneform $\mathrm{d} \mathcal{A} \neq 0$. By using the (32) we have imposed a condition for the solenoidal part of $\mathcal{A}$ but the lamellar part remains not specified. Using (32) the Maxwell's equation (29b) must be satisfied

$$
\mathrm{d}(\underline{\mathcal{E}}+j \omega \mathcal{A})=0
$$

which represents the exterior derivative of a one-form that due to the Poincaré's lemma must vanish. As mentioned before in general $\mathrm{d} \mathcal{A} \neq 0$, and therefore we introduce a scalar potential $\phi$. Since $\operatorname{dd} \phi=0$ we can express the $\underline{\mathcal{E}}$ as follows

$$
\underline{\mathcal{E}}=-j \omega \underline{\mathcal{A}}-\mathrm{d} \phi
$$


In such way the (33) is verified and in the following we will show that the not yet specified lamellar part must satisfy the Lorenz condition. By inserting the (32) and (34) into (29b)

$$
\mathrm{d} \star \mathrm{d} \mathcal{A}=j \omega \underline{\mu} \underline{\varepsilon} \star(-j \omega \underline{\mathcal{A}}-\mathrm{d} \phi)+\underline{\mu} \underline{\mathcal{I}}
$$

Applying the Hodge operator in both sides of (35) and considering that $\star \star=1$ we obtain

$$
\star \mathrm{d} \star \mathrm{d} \mathcal{A}=j \omega \underline{\mu} \underline{\varepsilon}(-j \omega \underline{\mathcal{A}}-\mathrm{d} \phi)+\underline{\mu} \star \underline{\mathcal{J}}
$$

Since $\star \mathrm{d} \star \mathrm{d} \mathcal{A}=\mathrm{d} \star \mathrm{d} \mathcal{A}$ we use the following identity

$$
\star \mathrm{d} \star \mathrm{d} \mathcal{A}=-\Delta \mathcal{A}+\mathrm{d} \star \mathrm{d} \star \mathcal{A}
$$

into the (36)

$$
-\Delta \mathcal{A}+\mathrm{d} \star \mathrm{d} \star \mathcal{A}=k^{2} \underline{\mathcal{A}}-j \omega \underline{\mu} \underline{\varepsilon} \mathrm{d} \phi+\underline{\mu} \star \underline{\mathcal{J}}
$$

the above equation can be simplified to satisfy by forcing the lamellar part of the vector potential one-form to satisfy the Lorenz condition as follows

$$
\mathrm{d} \star \mathrm{d} \star \mathcal{A}=-j \omega \underline{\mu} \underline{\underline{\varepsilon}} \mathrm{d} \phi
$$

rearranging the equation

$$
\mathrm{d}(\star \mathrm{d} \star \mathcal{A})=\mathrm{d}(-j \omega \underline{\mu} \underline{\varepsilon} \phi)
$$

which is satisfied if

$$
\phi=\frac{\star \mathrm{d} \star \mathcal{A}}{-j \omega \underline{\mu} \underline{\varepsilon}}
$$

With this choice of $\phi$, (35) is reduced to

$$
\Delta \mathcal{A}+k^{2} \underline{\mathcal{A}}=-\underline{\mu} \star \underline{\mathcal{J}}
$$

which represents the Helmholtz equation. Applying the Hodge operator and exterior derivative to both sides of (34), we define the divergence as follows

$$
\mathrm{d} \star \underline{\mathcal{E}}=-j \omega \mathrm{d} \star \underline{\mathcal{A}}-\mathrm{d} \star \mathrm{d} \phi
$$


form the (30b) and (31b) and applying the Hodge operator to both sides of the (43)

$$
-\frac{1}{\varepsilon} \star \underline{\mathcal{Q}}=-j \omega \star \mathrm{d} \star \underline{\mathcal{A}}-\star \mathrm{d} \star \mathrm{d} \phi
$$

Moreover considering that for a zero-form the Laplace operator $\Delta \phi=\star \mathrm{d} \star$ $\mathrm{d} \phi$, and from (41) we obtain the scalar Helmholtz equation

$$
\Delta \phi+k^{2} \phi=-\frac{1}{\underline{\varepsilon}} \star \underline{\mathcal{Q}}
$$

Finally, using the Lorenz condition (41), the field can be written in terms of vector potential form as follows:

$$
\begin{aligned}
& \underline{\mathcal{H}}=\frac{1}{\underline{\mu}} \star \mathrm{d} \mathcal{A} \\
& \underline{\mathcal{E}}=-j \omega \mathcal{A}+\frac{\mathrm{d} \star \mathrm{d} \star \mathcal{A}}{j \omega \underline{\underline{\mu}}}
\end{aligned}
$$

In regions where only magnetic sources are presents, considering the duality, the same arguments used for deriving the vector magnetic potential form $\mathcal{A}$ and the scalar magnetic potential zero-form $\phi$ can be used for deriving an electric vector potential $\mathcal{F}$ and scalar electric potential $\psi$. We obtain

$$
\begin{aligned}
\underline{\mathcal{E}} & =-\frac{1}{\underline{\varepsilon}} \star \mathrm{d} \mathcal{F} \\
\underline{\mathcal{H}} & =-j \omega \mathcal{F}+\frac{\mathrm{d} \star \mathrm{d} \star \mathcal{F}}{j \omega \underline{\underline{\mu}}}
\end{aligned}
$$

where $\underline{\mathcal{J}} \mathrm{m}$ denotes the impressed magnetic current phasor and $\underline{\mathcal{Q}} \mathrm{m}$ the magnetic charge density. If both currents are present, we may use a superposition and combining the fields part in terms of $\mathcal{A}$ and $\mathcal{F}$ as follows

$$
\begin{aligned}
& \underline{\mathcal{E}}=-j \omega \mathcal{A}+\frac{\mathrm{d} \star \mathrm{d} \star \mathcal{A}}{j \omega \underline{\underline{\varepsilon}}}-\frac{1}{\underline{\varepsilon}} \star \mathrm{d} \mathcal{F} \\
& \underline{\mathcal{H}}=\frac{1}{\underline{\mu}} \star \mathrm{d} \mathcal{A}-j \omega \mathcal{F}+\frac{\mathrm{d} \star \mathrm{d} \star \mathcal{F}}{j \omega \underline{\underline{\varepsilon}} \underline{\mu}}
\end{aligned}
$$





\section{Green's function for multilayered cylindrical radiating structures}

\subsection{Dyadic Green's function definition for multilayered cylindrical regions}

We consider now a circular cylindrical coordinates system $\rho, \varphi, z$. The coordinates are defined in the following interval:

$$
\begin{array}{cc}
u=\rho & 0 \leq \rho<+\infty \\
v=\varphi & 0 \leq \varphi<2 \pi \\
w=z & -\infty \leq z<+\infty
\end{array}
$$

Since the cylindrical coordinates form an orthonormal coordinates system, can be derived analytically directly from the Cartesian coordinates via the metric coefficients. In particular they are given as in [21] as following:

$$
\begin{array}{lll}
g_{1}=1 & g_{2}=\rho & g_{3}=1 \\
g_{1}^{\prime}=1 & g_{2}^{\prime}=\rho^{\prime} & g_{3}^{\prime}=1
\end{array}
$$

Therefore the unit-one forms are given as follows:

$$
\begin{array}{ccc}
s_{1}=\mathrm{d} \rho & s_{2}=\rho \mathrm{d} \varphi & s_{3}=\mathrm{d} z \\
s_{1}^{\prime}=\mathrm{d} \rho^{\prime} & s_{2}^{\prime}=\rho^{\prime} \mathrm{d} \varphi^{\prime} & s_{3}^{\prime}=\mathrm{d} z^{\prime}
\end{array}
$$

Considering (51) the expression of the Dirac current distribution is readily obtained from (5) as follows:

$\mathcal{J}\left(\mathbf{r}^{\prime}\right)=J_{0_{\rho^{\prime}}} \delta\left(\mathbf{r}-\mathbf{r}^{\prime}\right) \rho^{\prime} \mathrm{d} \varphi^{\prime} \wedge \mathrm{d} z^{\prime}+J_{0_{\varphi^{\prime}}} \delta\left(\mathbf{r}-\mathbf{r}^{\prime}\right) \mathrm{d} z^{\prime} \wedge \mathrm{d} \rho^{\prime}+J_{0_{z^{\prime}}} \delta\left(\mathbf{r}-\mathbf{r}^{\prime}\right) \rho^{\prime} \mathrm{d} \rho^{\prime} \wedge \mathrm{d} \varphi^{\prime}$ 


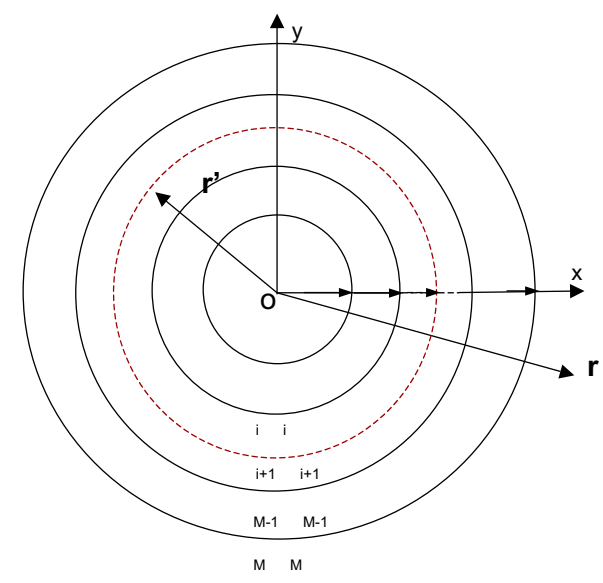

Figure 2.: Cylindrical multilayered structure

where

$$
\delta\left(\mathbf{r}-\mathbf{r}^{\prime}\right)=\frac{\delta\left(\rho-\rho^{\prime}\right) \delta\left(\varphi-\varphi^{\prime}\right) \delta\left(z-z^{\prime}\right)}{\rho^{\prime}}
$$

since

$$
\int_{V^{\prime}} \frac{\delta\left(\rho-\rho^{\prime}\right) \delta\left(\varphi-\varphi^{\prime}\right) \delta\left(z-z^{\prime}\right)}{\rho^{\prime}} \mathrm{d} \rho^{\prime} \wedge \rho^{\prime} \mathrm{d} \varphi^{\prime} \wedge \mathrm{d} z^{\prime}=1
$$

Similarly, using (51) in (4) we obtain the Green's double one form for cylindrical coordinates

$$
\begin{array}{r}
\mathcal{G}_{e}\left(\mathbf{r}, \mathbf{r}^{\prime}\right)=G_{\rho \rho^{\prime}} \mathrm{d} \rho \mathrm{d} \rho^{\prime}+\rho^{\prime} G_{\rho \varphi^{\prime}} \mathrm{d} \rho \mathrm{d} \varphi^{\prime}+G_{\rho z^{\prime}} \mathrm{d} \rho \mathrm{d} z^{\prime} \\
+\rho G_{\varphi \rho^{\prime}} \mathrm{d} \varphi \mathrm{d} \rho^{\prime}+\rho \rho^{\prime} G_{\varphi \varphi^{\prime}} \mathrm{d} \varphi \mathrm{d} \varphi^{\prime}+\rho G_{\varphi z^{\prime}} \mathrm{d} \varphi \mathrm{d} z^{\prime} \\
+G_{z \rho^{\prime}} \mathrm{d} z \mathrm{~d} \rho^{\prime}+\rho^{\prime} G_{z \varphi^{\prime}} \mathrm{d} z \mathrm{~d} \varphi^{\prime}+G_{z z^{\prime}} \mathrm{d} z \mathrm{~d} z^{\prime}
\end{array}
$$

Using (51) in (7) the equation (8), (9) and (10) are readily obtained form the general case. We consider a circular cylindrically multilayered lossless structure of $M$ layers, infinitely extended in $z$ direction as depicted in Fig. 2. The double one form (55) can be directly used. If the source is placed at the layer $j$ and the observation point is at the layer $i$ with $1 \leq i, j \leq M$ 
then $(10)$ can be directly written as follows:

$$
G_{p q}^{i j}\left(\mathbf{r}, \mathbf{r}^{\prime}\right)=\left.E_{p}^{i}(\mathbf{r})\right|_{\mathcal{J}_{j}(\mathbf{r})=\delta\left(\mathbf{r}-\mathbf{r}^{\prime}\right) \mathbf{u}_{q}}
$$

In the following section a procedure for deriving the scalar components of the dyadic Green's function is introduced.

\subsubsection{Computational Method}

The derivation of the Green's function starts by modeling the source-free cylindrical layers. We assume and neglect a time harmonic dependence of the field $e^{j \omega t}$. The electromagnetic field distribution for the geometry of Figure 2 can be obtained in terms of $z$-directed transverse magnetic form $(T M) \mathcal{A}=\mathrm{d} z \psi_{\mathcal{A}}$, and transverse electric vector potential form $(T E) \mathcal{F}=$ $\mathrm{d} z \psi_{\mathcal{F}}$. For the vector potential form Ansatz we observe that the structure is translational invariant in $z$ since is infinitely extended. Based on that we may build the solution for each mode considering that the propagation along $z$ has an identical z-dependency of the type $e^{-j k_{z} z}$ for each mode. Since the dependency is identical also for all the cylindrical layers $1<i<M$, we reduce the problem introducing an eigenfunction expansion in $z$ direction using a Fourier integral in the usual way $[7,32]$

$$
\left\{\begin{array}{c}
\mathcal{A}^{i} \\
\mathcal{F}^{i}
\end{array}\right\}=\mathrm{d} z \int_{k_{z}}\left\{\begin{array}{l}
\bar{\psi}_{\mathcal{A}}^{i}\left(\rho, \rho^{\prime}, \varphi, \varphi^{\prime}, k_{z}\right) \\
\bar{\psi}_{\mathcal{F}}^{i}\left(\rho, \rho^{\prime}, \varphi, \varphi^{\prime}, k_{z}\right)
\end{array}\right\} e^{-j k_{z}\left(z-z^{\prime}\right)} d k_{z}
$$

where the Fourier transformations are performed at the point $\mathbf{r}^{\prime}=\left(\rho^{\prime}, \varphi^{\prime}, z^{\prime}\right)$ inside the cylindrical structure. Analogous considerations can be made for the $\varphi$-dependency of the solution which is rotational invariant. As it stated in Section 6.1 in this case the $\varphi$-dependency of the solution is bounded from $0<\varphi<2 \pi$. Therefore, while $k_{z}$ assumes a continuum of values, $n$ is forced to assume discrete values. In particular, if we require for each mode that $\psi_{\mathcal{A}, \mathcal{F}}^{i}(\varphi)=\psi_{\mathcal{A}, \mathcal{F}}^{i}(\varphi+2 \pi)$, then $n$ is forced to be integer. Therefore, we reduce the problem expanding in eigenfunctions using a Fourier series

$$
\left\{\begin{array}{l}
\mathcal{A}^{i} \\
\mathcal{F}^{i}
\end{array}\right\}=\mathrm{d} z \frac{j}{8 \pi} \sum_{n=0}^{+\infty} \int_{k_{z}}\left\{\begin{array}{c}
\bar{\psi}_{\mathcal{A}}^{i}\left(\rho, \rho^{\prime} n, k_{z}\right) \\
\bar{\psi}_{\mathcal{F}}^{i}\left(\rho, \rho^{\prime} n, k_{z}\right)
\end{array}\right\} e^{j n\left(\varphi-\varphi^{\prime}\right)} e^{-j k_{z}\left(z-z^{\prime}\right)} d k_{z}
$$


The $\bar{\psi}_{\mathcal{A}, \mathcal{F}}^{i}$ describe the radial dependency of the $T M$ and $T E$ potential respectively and must be a solution of the radial boundary problem described by the radial component of the homogeneous wave equation which in cylindrical coordinates assumes the following form:

$$
\left[\frac{1}{\rho} \frac{d}{d \rho}\left(\rho \frac{d}{d \rho}\right)-\frac{n^{2}}{\rho^{2}}+k_{\rho_{i}}^{2}\right] \bar{\psi}_{\mathcal{A}, \mathcal{F}}^{i}=0
$$

where $k_{\rho_{i}}$ is the propagation constant at the layer $i$ in $\rho$ direction and $k_{z}$ in $z$ direction, with $k_{i}^{2}=k_{\rho_{i}}^{2}+k_{z}^{2}$. In a radiation problem with excitation confined inside the cylindrical structure cylindrical wave traveling outward (along $+\rho$ ) and inward (along $-\rho$ ) are expected. The solution of the radially boundary problem is therefore described in spectral domain by superposition of cylindrical waves as follows

$$
\begin{aligned}
& \bar{\psi}_{\mathcal{F}}^{i}=\sum_{n=0}^{\infty}\left[A_{n}^{i} H_{n}^{(1)}\left(k_{\rho_{i}} \rho\right)+B_{n}^{i} H_{n}^{(2)}\left(k_{\rho_{i}} \rho\right)\right] \\
& \bar{\psi}_{\mathcal{A}}^{i}=\sum_{n=0}^{\infty}\left[C_{n}^{i} H_{n}^{(1)}\left(k_{\rho_{i}} \rho\right)+D_{n}^{i} H_{n}^{(2)}\left(k_{\rho_{i}} \rho\right)\right]
\end{aligned}
$$

where $A_{n}^{i}, B_{n}^{i}, C_{n}^{i}, D_{n}^{i}$ are unknown modal coefficients that will be determined from the boundary conditions, $k_{\rho_{i}}$ is the propagation constant at the layer $i$ in $\rho$ direction and $k_{z}$ in $z$ direction, with $k_{i}^{2}=k_{\rho_{i}}^{2}+k_{z}^{2}$. They physically represent the waves amplitudes of the inward and outward cylindrical waves described by $H_{n}^{(1)}\left(k_{\rho_{i}} \rho\right)$ and $H_{n}^{(2)}\left(k_{\rho_{i}} \rho\right)$, which denote Hankel functions of the first and second kind respectively. We observe that due to the symmetry of the structure we have reduced the problem to one-dimensional homogeneous Sturm-Liouville problem which the solution is given by a combination of cylindrical Hankel functions. That will insure the completeness of the solution after the introduction of the Dirac sources. We note that in using Hankel functions a proper normalization factor must be introduced to insure numerical stability.

\subsubsection{Parametric description of the cylindrical layer}

In the following a parametric description of the cylindrical layers is introduced. We start modeling the cylindrical interface. The $T E$ and $T M$ modes are generally coupled at the cylindrical interface for $k_{z} \neq 0$ and therefore 
cannot be studied separately. Considering that, from (60), as a function of the unknown modal coefficients, the $n$th harmonic component of the tangential field at the cylindrical interface is given in spectral domain as superposition of $T E$ and $T M$ cylindrical waves as follows:

$$
\begin{aligned}
& \bar{E}_{z_{n}}^{i}\left(n, k_{z}, \rho\right)=\frac{k_{\rho_{i}}^{2}}{j \omega \epsilon_{i}} \bar{\psi}_{\mathcal{A}}^{i} \quad \bar{H}_{\varphi_{n}}^{i}\left(n, k_{z}, \rho\right)=-\frac{\partial \bar{\psi}_{\mathcal{A}}^{i}}{\partial \rho}+\frac{j n k_{z}}{j \omega \mu_{i} \rho} \bar{\psi}_{\mathcal{F}}^{i} \\
& \bar{H}_{z_{n}}^{i}\left(n, k_{z}, \rho\right)=\frac{k_{\rho_{i}}^{2}}{j \omega \mu_{i}} \psi_{\mathcal{F}}^{i} \quad \bar{E}_{\varphi_{n}}^{i}\left(n, k_{z}, \rho\right) \quad=\frac{j k_{z} n}{j \omega \epsilon_{i} \rho} \bar{\psi}_{\mathcal{A}}^{i}+\frac{\partial \psi_{F}^{i}}{\partial \rho}
\end{aligned}
$$

Considering that the fields are represented by linear combinations of partial waves, described by the coefficients $A_{n}^{i}, B_{n}^{i}, C_{n}^{i}, D_{n}^{i}$, we introduce a matrix notation of (61) for each boundary surface as follows:

$$
\left(\begin{array}{c}
\bar{E}_{z_{n}}^{i}\left(n, k_{z}, \rho\right) \\
\bar{H}_{\varphi_{n}}^{i}\left(n, k_{z}, \rho\right) \\
\bar{E}_{\varphi_{n}}^{i}\left(n, k_{z}, \rho\right) \\
\bar{H}_{z_{n}}^{i}\left(n, k_{z}, \rho\right)
\end{array}\right)=\mathbf{M}^{i}\left(\rho, n, k_{\rho_{i}}\right)_{<4 \times 4>}\left(\begin{array}{c}
A_{n}^{i} \\
B_{n}^{i} \\
C_{n}^{i} \\
D_{n}^{i}
\end{array}\right)
$$

where $\mathbf{M}^{i}$ is a $4 \times 4$ matrix given as follows:

$$
\mathbf{M}^{i}=\left(\begin{array}{cccc}
0 & 0 & \frac{k_{\rho_{i}}^{2}}{j \omega \omega_{i}} H_{n}^{(1)}\left(\zeta_{i}\right) & \frac{k_{\rho_{i}}^{2}}{j \omega \epsilon_{i}} H_{n}^{(2)}\left(\zeta_{i}\right) \\
-H_{n}^{(1)^{\prime}}\left(\zeta_{i}\right) & -H^{(2)^{\prime}}\left(\zeta_{i}\right) & \frac{j n k_{z}}{j \omega \mu_{i} \rho} H_{n}^{(1)}\left(\zeta_{i}\right) & \frac{j n k_{z}}{j \omega \mu_{i} \rho} H_{n}^{(2)}\left(\zeta_{i}\right) \\
-H_{n}^{(1)^{\prime}}\left(\zeta_{i}\right) & -H_{n}^{(2)^{\prime}}\left(\zeta_{i}\right) & -\frac{j n k_{z}}{j \omega \epsilon_{i} \rho} H_{n}^{(1)}\left(\zeta_{i}\right) & -\frac{j n k_{z}}{j \omega \epsilon_{i} \rho} H_{n}^{(2)}\left(\zeta_{i}\right) \\
\frac{k_{\rho_{i}}^{2}}{j \omega \epsilon_{i}} H_{n}^{(1)}\left(\zeta_{i}\right) & \frac{k_{\rho_{i}}^{2}}{j \omega \epsilon_{i}} H_{n}^{(2)}\left(\zeta_{i}\right) & 0 & 0
\end{array}\right)
$$

where $\zeta_{i}=k_{\rho_{i}} \rho$. Each cylindrical layer is completely described by the tangential components at its boundary surfaces. Considering this and using (62), we obtain a parametric model of the layer in the following way:

$$
\left(\begin{array}{c}
\mathbf{F}^{+} \\
\mathbf{F}^{-}
\end{array}\right)=\left(\begin{array}{c}
\mathbf{M}^{i^{+}} \\
\mathbf{M}^{i^{-}}
\end{array}\right)_{<8 \times 4>}\left(\begin{array}{c}
A_{n}^{i} \\
B_{n}^{i} \\
C_{n}^{i} \\
D_{n}^{i}
\end{array}\right)
$$




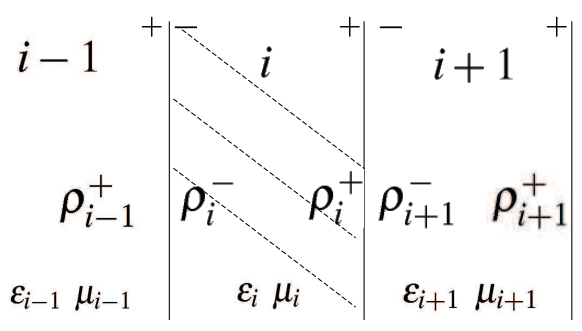

Figure 3.: Layers description

where

$$
\begin{aligned}
& \mathbf{F}^{+}=\left(\bar{E}_{z_{n}}^{i^{+}}\left(n, k_{z}, \rho_{i}^{+}\right), \bar{H}_{\varphi_{n}}^{i^{+}}\left(n, k_{z}, \rho_{i}^{+}\right), \bar{E}_{\varphi_{n}}^{i^{+}}\left(n, k_{z}, \rho_{i}^{+}\right), \bar{H}_{z_{n}}^{i^{+}}\left(n, k_{z}, \rho_{i}^{+}\right)\right)^{T} \\
& \mathbf{F}^{-}=\left(\bar{E}_{z_{n}}^{i^{-}}\left(n, k_{z}, \rho_{i}^{-}\right), \bar{H}_{\varphi_{n}}^{i^{-}}\left(n, k_{z}, \rho_{i}^{-}\right), \bar{E}_{\varphi_{n}}^{i^{-}}\left(n, k_{z}, \rho_{i}^{-}\right), \bar{H}_{z_{n}}^{i^{-}}\left(n, k_{z}, \rho_{i}^{-}\right)\right)^{T}
\end{aligned}
$$

and with " - " and " + " we name the inner side and outer side of the boundary surface respectively as depicted in Fig. 3. Once a parametric description of the layer is obtained, the connection between the layers is given by the continuity condition of the tangential components, at the inner and outer boundary surface. A more detailed description is introduced in the Section 6.1.4.

\section{Parametric description of the innermost and outermost layer}

We consider now the innermost $(i=1)$ and outermost $(\mathrm{i}=\mathrm{M})$ layers. Since the structure is extended $\rho>0$, for $\rho=0$ the finiteness condition must be fulfilled for the layer $i=1$.

Since $H_{n}^{(1)}$ and $H_{n}^{(2)}$ are singular at $\rho=0$, we choose from (60) $A_{n}^{1}=B_{n}^{1}$ and $C_{n}^{1}=D_{n}^{1}$. In this way, by using the following linear relations between Hankel functions

$$
\begin{aligned}
& H_{n}^{(1)}\left(k_{\rho_{1}} \rho\right)=J_{n}\left(k_{\rho_{1}} \rho\right)+j Y_{n}\left(k_{\rho_{1}} \rho\right) \\
& H_{n}^{(2)}\left(k_{\rho_{1}} \rho\right)=J_{n}\left(k_{\rho_{1}} \rho\right)-j Y_{n}\left(k_{\rho_{1}} \rho\right)
\end{aligned}
$$


we obtain a particular potential Ansatz for the innermost layer

$$
\begin{aligned}
\bar{\psi}_{\mathcal{F}}^{i} & =2 A_{n}^{1} J_{n}\left(k_{\rho_{1}} \rho\right) \\
\bar{\psi}_{\mathcal{A}}^{i} & =2 C_{n}^{1} J_{n}\left(k_{\rho_{1}} \rho\right)
\end{aligned}
$$

Therefore, using (68) into (61), (63) becomes

$$
\mathbf{M}_{<4 \times 2>}^{1}=\left(\begin{array}{cc}
2 \frac{k_{\rho_{1}}^{2}}{j \omega \varepsilon_{1}} J_{n}\left(k_{\rho_{1}} \rho\right) & 0 \\
-2 J_{n}^{\prime}\left(k_{\rho_{1}} \rho\right) & 2 \frac{j n k_{z}}{j \omega \mu_{1} \rho} J_{n}\left(k_{\rho_{1}} \rho\right) \\
2 \frac{j n k_{z}}{j \omega \varepsilon_{1} \rho} J_{n}\left(k_{\rho_{1}} \rho\right) & 2 J_{n}^{\prime}\left(k_{\rho_{1}} \rho\right) \\
0 & 2 \frac{k_{\rho_{1}}}{j \omega \mu_{1}} J_{n}\left(k_{\rho_{1}} \rho\right)
\end{array}\right)
$$

Considering the outermost layer as free space region, only outgoing waves exist vanishing at $\rho=+\infty$ (radiation condition) and therefore from (60) $A_{n}^{M}=0$ and $C_{n}^{M}=0$. In this case the particular potential Ansatz for the outermost region is given by the following:

$$
\begin{aligned}
\bar{\psi}_{\mathcal{F}}^{i} & =B_{n}^{M} H_{n}^{(2)}\left(k_{\rho_{M}} \rho\right) \\
\bar{\psi}_{\mathcal{A}}^{i} & =D_{n}^{M} H_{n}^{(2)}\left(k_{\rho_{M}} \rho\right)
\end{aligned}
$$

Accordingly, using (70) into (61), the (63) becomes

$$
\mathbf{M}_{<4 \times 2>}^{1}=\left(\begin{array}{cc}
\frac{k_{\rho_{M}}^{2}}{\frac{j \omega \varepsilon_{M}}{H_{n}}(2)}\left(k_{\rho_{M}} \rho\right) & 0 \\
-H_{n}^{(2)^{\prime}}\left(k_{\rho_{M}} \rho\right) & \frac{j n k_{z}}{j \omega \mu_{M} \rho} H_{n}^{(2)}\left(k_{\rho_{M}} \rho\right) \\
\left.\frac{j n k_{z}}{j \omega \varepsilon_{M} \rho} H_{n}^{(2)}\left(k_{\rho_{M}} \rho\right) \rho\right) & H_{n}^{(2)^{\prime}}\left(k_{\rho_{M}} \rho\right) \\
0 & \frac{k_{\rho_{1}}}{j \omega \mu_{M}} H_{n}^{(2)}\left(k_{\rho_{M}} \rho\right)
\end{array}\right)
$$

\section{Description of metallic layers}

In this Section a parametric model for Perfect Electric Conducting Cylindrical (PEC) surfaces will be derived. For radiating problems, the source can be always assumed to be external to the PEC. We compute the field using the equivalence principle and the impressed electrical polarization [21]. The electric impressed polarization area on the surface of the PEC, Figure 4, is related to the tangential components $\underline{\mathcal{H}}_{t}$ of the magnetic field form in the following way:

$$
\left.\mathcal{M}_{e A}(\mathbf{r})=\frac{1}{j \omega} \mathrm{d} \rho\right\lrcorner(\mathrm{d} \rho \wedge \underline{\mathcal{H}})=\frac{1}{j \omega} \underline{\mathcal{H}}_{t}=\frac{1}{j \omega}\left(H_{z} \rho \mathrm{d} \varphi+H_{\varphi} \mathrm{d} z\right)
$$




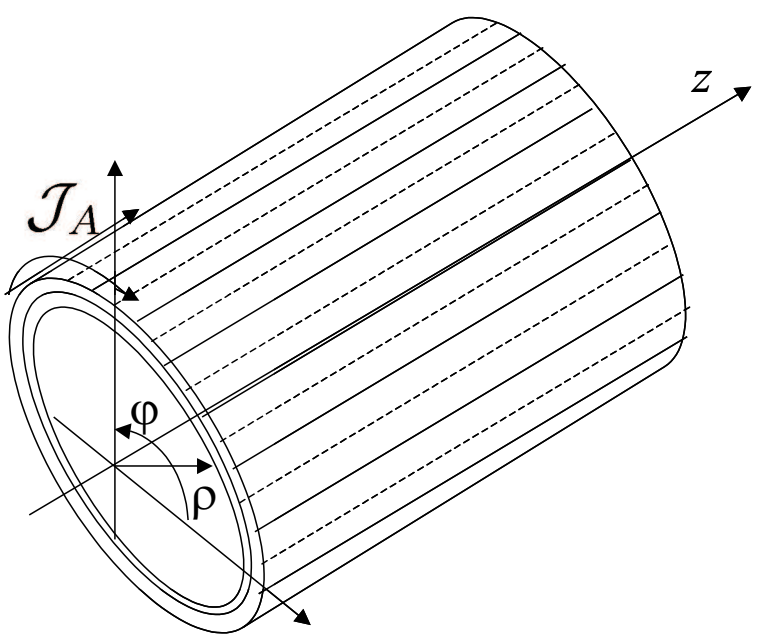

Figure 4.: PEC

Since inside the PEC the fields vanish, for an impressed polarization area the tangential magnetic field can be univocally determined. The electric surface polarization form is related to the surface density form in the following way:

$$
\mathcal{M}_{e A}\left(\mathbf{r}_{s}\right)=j \omega \mathcal{J}_{A}
$$

In case of circular cylindrical coordinate the surface current density can be written by as following.

$$
\mathcal{J}_{A}=J_{A_{\varphi}}(\varphi, z) \rho \mathrm{d} \varphi-J_{A_{z}}(\varphi, z) \mathrm{d} z
$$

At $\mathbf{r}_{s}$, i.e. at the boundary surface of the PEC,

$$
\mathcal{M}_{e A}\left(\mathbf{r}_{s}\right)=j \omega\left(J_{A_{\varphi}}\left(\varphi_{s}, z_{s}\right) \rho_{s} \mathrm{~d} \varphi-J_{A_{z}}\left(\varphi_{s}, z_{s}\right) \mathrm{d} z\right)
$$

Inserting (75) into (72) at $\mathbf{r}_{s}$, we obtain

$$
H_{z} \rho_{s} \mathrm{~d} \varphi+H_{\varphi} \mathrm{d} z=J_{A_{\varphi}}\left(\varphi_{s}, z_{s}\right) \rho_{s} \mathrm{~d} \varphi-J_{A_{z}}\left(\varphi_{s}, z_{s}\right) \mathrm{d} z
$$


According to (76) we obtain the value for the tangential magnetic components on the PEC

$$
\begin{aligned}
H_{z} & =J_{A_{\varphi}}\left(\varphi_{s}, z_{s}\right) \\
H_{\varphi} & =-J_{A_{z}}\left(\varphi_{s}, z_{s}\right)
\end{aligned}
$$

Next we define the boundary condition in spectral domain. A convenient description is possible, if the spatial distribution of the currents is separable in $\rho, \varphi$ and $z$. We assume also the current symmetric in $\varphi$. In this case the currents are sheets of currents of the form:

$$
\left\{\begin{array}{c}
J_{A_{z}}(\varphi, z) \\
J_{A_{\varphi}}(\varphi, z)
\end{array}\right\}=\left\{\begin{array}{c}
J_{0_{z}} \mathrm{~d} z \\
J_{0_{\varphi}} \rho \mathrm{d} \varphi
\end{array}\right\} J_{z}(z) J_{\varphi}(\varphi) .
$$

Such a current distribution can be represented in spectral domain defining a Fourier transformation and expansion in $z$ and $\varphi$ direction respectively as follows:

$$
\left\{\begin{array}{c}
J_{A_{z}}(\varphi, z) \\
J_{A_{\varphi}}(\varphi, z)
\end{array}\right\}=\left\{\begin{array}{c}
J_{0_{z}} \mathrm{~d} z \\
J_{0_{\varphi}} \rho \mathrm{d} \varphi
\end{array}\right\} \frac{j}{8 \pi} \sum_{n=0}^{+\infty} J_{\varphi}(n) e^{-j n\left(\varphi-\varphi^{\prime}\right)} \int_{k_{z}} J_{k_{z}}\left(k_{z}\right) e^{j k_{z}\left(z-z^{\prime}\right)} d k_{z} .
$$

where the spectral component of the current density sources can be described as

$$
\left\{\begin{array}{c}
\bar{J}_{A_{z}}\left(n, k_{z}\right) \\
\bar{J}_{A_{\varphi}}\left(n, k_{z}\right)
\end{array}\right\}=\left\{\begin{array}{c}
J_{0_{z}} \mathrm{~d} z \\
J_{0_{\varphi}} \rho \mathrm{d} \varphi
\end{array}\right\} J_{\varphi}(n) J_{k_{z}}\left(k_{z}\right) .
$$

Therefore, the magnetic field component can be determined in SD as follows

$$
\begin{aligned}
\bar{H}_{z_{n}}\left(n, k_{z}, \rho_{s}\right) & =J_{0_{\varphi}} J_{\varphi}(n) J_{k_{z}}\left(k_{z}\right) \\
\bar{H}_{\varphi_{n}}\left(n, k_{z}, \rho_{s}\right) & =-J_{0_{z}} J_{z}(n) J_{k_{z}}\left(k_{z}\right)
\end{aligned}
$$

when $J_{z}(z)=\delta(z)$ and $J_{\varphi}(\varphi)=\delta(\varphi)$ then $J_{k_{z}}\left(k_{z}\right)=1$ and $J_{n}(n)=1 / \pi$ for $n=0,1,2 \ldots$ and (81) becomes

$$
\begin{aligned}
\bar{H}_{z_{n}}\left(n, k_{z}, \rho_{s}\right) & =\frac{J_{0_{\varphi}}}{\pi} \\
\bar{H}_{\varphi_{n}}\left(n, k_{z}, \rho_{s}\right) & =-J_{0_{z}}
\end{aligned}
$$

From (82) we may note that cylindrical metallic boundary $T E$ and $T M$ waves are uncoupled since if $J_{A_{\varphi}}=0$ there is no $\bar{H}_{z}$ and/or $J_{A_{z}}=0$ there 
is no $\bar{H}_{\varphi}$. Since there are no impressed magnetic sources

$$
\left.\mathcal{M}_{m A}(\mathbf{r})=\frac{1}{j \omega} \mathrm{d} \rho\right\lrcorner(\mathrm{d} \rho \wedge \underline{\mathcal{E}})=0
$$

and consequently for $T E$ and $T M$ waves

$$
\begin{aligned}
& \bar{E}_{z_{n}}\left(n, k_{z}, \rho_{s}\right)=0 \\
& \bar{E}_{\varphi_{n}}\left(n, k_{z}, \rho_{s}\right)=0
\end{aligned}
$$

Considering that a parametric description of the $i$ boundary surface for a $\mathrm{PEC}$ can be introduced in the following way

$$
\left(\begin{array}{l}
\bar{E}_{z_{n}}^{i}\left(n, k_{z}, \rho_{s}\right) \\
\bar{H}_{\varphi_{n}}^{i}\left(n, k_{z}, \rho_{s}\right) \\
\bar{E}_{\varphi_{n}}^{i}\left(n, k_{z}, \rho_{s}\right) \\
\bar{H}_{z_{n}}^{i}\left(n, k_{z}, \rho_{s}\right)
\end{array}\right)=\left(\begin{array}{cc}
0 & 0 \\
0 & J_{0_{z}} \\
0 & 0 \\
-J_{0_{\varphi}} / \pi & 0
\end{array}\right)\left(\begin{array}{c}
B_{n}^{i} \\
D_{n}^{i}
\end{array}\right)
$$

\subsubsection{Source modelling}

In the previous Sections we have derived a parametric representation of the cylindrical boundary surfaces and layers. In the following section, similarly to Section 6.1.2, we introduce the modelling of the sources. For this purpose the currents flowing in a conformal interface layer between two adjacent homogenous dielectric layers must be described in the spectral domain. In the following section only $z$-oriented currents are considered but the result is readily usable also for $\varphi$-oriented sources. Also in this case a convenient description is possible, if the spatial distribution of the currents is separable in $\rho, \varphi$ and $z$. We assume also current symmetry in $\varphi$ and $\varphi^{\prime}=0, z^{\prime}=0$. In this case the current are sheet of currents of the form:

$$
\begin{gathered}
\mathbf{J}(\rho, \varphi, z)=\delta\left(\rho-\rho^{\prime}\right) \mathrm{d} \rho \wedge J_{A}(\varphi, z)= \\
\rho \mathrm{d} \rho \wedge \mathrm{d} \varphi J_{0_{z}} \delta\left(\rho-\rho^{\prime}\right) J_{z}(z) J_{\varphi}(\varphi)+\mathrm{d} z \wedge \mathrm{d} \rho J_{0_{\varphi}} \delta\left(\rho-\rho^{\prime}\right) J_{z}(z) J_{\varphi}(\varphi) .
\end{gathered}
$$

As previously, such a current density distribution form can be represented in spectral domain defining a Fourier transformation and expansion in $z$ 
and $\varphi$ directions respectively as follows:

$$
\begin{gathered}
\mathbf{J} \rho, \varphi, z)=\rho \mathrm{d} \rho \wedge \mathrm{d} \varphi \frac{j}{8 \pi} \sum_{n=0}^{+\infty} J_{\varphi}(n) \cos (n \varphi) \int_{k_{z}} J_{0_{z}} \delta\left(\rho-\rho^{\prime}\right) J_{k_{z}}\left(k_{z}\right) e^{j k_{z} z} \mathrm{~d} k_{z}+ \\
\mathrm{d} z \wedge \mathrm{d} \rho \frac{j}{8 \pi} \sum_{n=0}^{+\infty} J_{\varphi}(n) \cos (n \varphi) \int_{k_{z}} J_{0_{z}} \delta\left(\rho-\rho^{\prime}\right) J_{k_{z}}\left(k_{z}\right) e^{j k_{z} z} \mathrm{~d} k_{z}
\end{gathered}
$$

where, in this case, the cosine dependency in $\varphi$ (rather than the more general exponential dependency) reflects the symmetry of the current distribution with respect to the angle. Therefore the spectral component of the current can be described as

$$
\begin{array}{r}
\bar{J}\left(n, k_{z}, \rho\right)=\rho \mathrm{d} \rho \wedge \mathrm{d} \varphi J_{0_{\varphi}} \delta\left(\rho-\rho^{\prime}\right) J_{\varphi}(n) J_{k_{z}}\left(k_{z}\right)+ \\
\rho \mathrm{d} z \wedge \mathrm{d} \rho J_{0_{z}} \delta\left(\rho-\rho^{\prime}\right) J_{\varphi}(n) J_{k_{z}}\left(k_{z}\right)
\end{array}
$$

Furthermore, since the radial dependence of the current distribution is a Dirac distribution, a transition from three-dimensional current density to sheet current density can be done considering that

$$
\overline{\mathbf{J}}_{A}\left(n, k_{z}\right)=\int_{\rho_{1}}^{\rho_{2}} \delta\left(\rho-\rho^{\prime}\right) \mathrm{d} \rho \wedge \bar{J}_{A}\left(n, k_{z}\right)
$$

\subsubsection{Interface Conditions}

We now investigate an interface between layer $i$ and layer $i+1$ for $1<i<$ $M-2$. The interface has the radius coordinate $\rho=\rho_{i}^{+}=\rho_{i+1}^{-}$. For an interface without impressed currents the tangential fields must be continuous. This can be formulated easily

$$
\left(\begin{array}{c}
\bar{E}_{\varphi_{n}, i+1}^{-} \\
\bar{H}_{z_{n}, i+1}^{-} \\
\bar{E}_{z_{n}, i+1}^{-} \\
-\bar{H}_{\varphi_{n}, i+1}^{-}
\end{array}\right)-\left(\begin{array}{c}
\bar{E}_{\varphi, i}^{+} \\
\bar{H}_{z_{n}, i}^{+} \\
\bar{E}_{z_{n}, i}^{+} \\
-\bar{H}_{\varphi_{n}, i}^{+}
\end{array}\right)=\left(\begin{array}{l}
0 \\
0 \\
0 \\
0
\end{array}\right)
$$




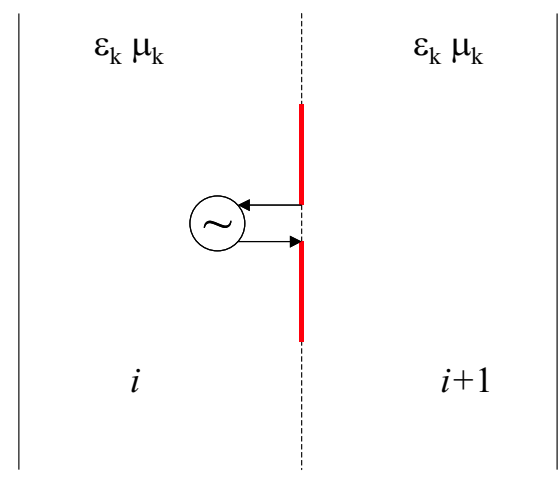

Figure 5.: Source in an homogeneous layer

If electric sheets of currents $J_{s, z,(i, i+1)}$ and $J_{s, \varphi,(i, i+1)}$ are impressed at the interface between layers $i$ and $i+1$, we must write

$$
\left(\begin{array}{c}
\bar{E}_{\varphi_{n}, i+1}^{+} \\
\bar{H}_{z_{n}, i+1}^{+} \\
\bar{E}_{z_{n}, i+1}^{+} \\
-\bar{H}_{\varphi_{n}, i+1}^{+}
\end{array}\right)-\left(\begin{array}{c}
\bar{E}_{\varphi_{n}, i}^{-} \\
\bar{H}_{z_{n}, i}^{-} \\
\bar{E}_{z_{n}, i}^{-} \\
-\bar{H}_{\varphi_{n}, i}^{-}
\end{array}\right)=\left(\begin{array}{c}
0 \\
-\bar{J}_{s, \varphi,(i, i+1)} \\
0 \\
-\bar{J}_{s, z,(i, i+1)}
\end{array}\right)
$$

We note that in such a representation the sources are always located at the interfaces between two dielectric layers. We consider the case in which the source is surrounded by a homogenous material. In this case we split the region in two different layers having the same properties in such way that the source is placed at the interface Fig. 5.

After the definition of the boundary conditions the electromagnetic problem is complectly defined. By applying systematically (91) at each boundary surface we obtain a linear system of equation of unknown modal coefficients $A_{n}^{i}, B_{n}^{i}, C_{n}^{i}$ and $D_{n}^{i}$. In the following section we will described the structure of the system of equations and discussed the uniqueness of the solution. 


\subsubsection{Calculation of the boundary conditions}

We consider the structure of Fig. 2 composed by $M$ layers. Each layer $1<i<M$ contribute with four unknown modal coefficients. The innermost layer $i=1$ either is metal or dielectric, contributes two unknown modal coefficients. The outermost layer $i=M$ either is infinitely extended or metallic boundary or free-space contributes with two unknown modal coefficients. The rest of the layers i.e. $M-2$ layers, contribute with four unknown coefficients. Therefore the number $N$ of unknown modal coefficients is $N=2+4(M-2)+2=4(M-1)$. On the other hands, by the boundary conditions every layers contributes with four equations and there are $M-1$ layers. Consequently the number of equations $E$ is $E=4(M-1)$ which is equal to $N$. Moreover, we may observe that, if the system is not singular the uniqueness of the solution is also verified.

\section{Numerical solution of the boundary conditions}

We investigate the property of the solution. As it is stated before, by applying systematically (91) at each boundary surface we obtain a system of $4(M-1)$ equations which we can rewrite in matrix notation as follows

$$
\mathbf{L C}=\overline{\mathbf{J}}
$$

where with $L$ we indicate the matrix of all the boundary surfaces

$$
\mathbf{L}=\left(\begin{array}{ccccc}
\mathbf{B}_{1}-\mathbf{B}_{2}^{-} & \mathbf{0} & \cdots & & \mathbf{0} \\
\mathbf{0} & \mathbf{B}_{2}^{+}-\mathbf{B}_{3}^{-} & & & \vdots \\
& \ddots & & & \\
\vdots & & \mathbf{B}_{i}^{+}-\mathbf{B}_{i+1}^{-} & & \\
& & & \ddots & \\
& & & \ddots & \\
\mathbf{0} & \mathbf{0} & \cdots & & \mathbf{B}_{M-1}^{+}-\mathbf{B}_{M}^{-}
\end{array}\right)
$$


where $\mathbf{B}=\mathbf{M}^{i}\left(\rho^{+}\right)-\mathbf{M}^{i+1}\left(\rho^{-}\right), \mathbf{C}$ a vector containing the unknown coefficients of all the layers

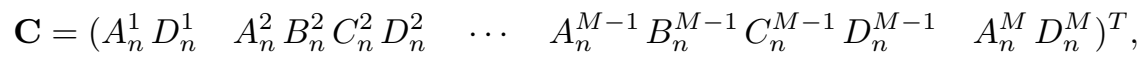

and $\overline{\mathbf{J}}$ is the source vector given by

$$
\overline{\mathbf{J}}=\left(0 \cdots J_{s, q(i, i+1)} \cdots 0\right)^{T}
$$

The system (92) can be directly solved to calculate the coefficient C. In solving (92) using standard matrix inversion, severe numerical problems due to the singular behavior of the Hankel functions may occur. A more detailed treatment of such problems is given in the Appendix C.

\subsection{Network description}

Transmission line models describe a field distribution in a wave-guiding structure in terms of mode amplitudes. There are two basically different approaches for describing a transmission line network in terms of wave amplitudes. In the first method, all transmission lines are characterized by their characteristic impedance. This results in a very simple matrix description (e.g. a $S$ or $T$ matrix) for the transmission line itself. In particular, reflection elements (e.g. $S_{11}$ and $S_{22}$ ) are zero in such a description. However, if transmission lines of different characteristic impedance are connected, a complicated connection matrix must be introduced. Second, the reference impedance at all the ports is set to a fixed value. The matrix description for transmission lines with a characteristic impedance not identical to the global reference impedance is rather complicated. However the connection of several line elements can be described in a rather simple way. In conclusion, line elements with different impedance cause a relatively complicated description either in the line or in the connection matrix. In the following section a network description for the cylindrical layered structure in the spectral domain will be derived as the parametric description of the cylindrical introduce in the section (6.1.2). Such a representation follows the second approach described above. 


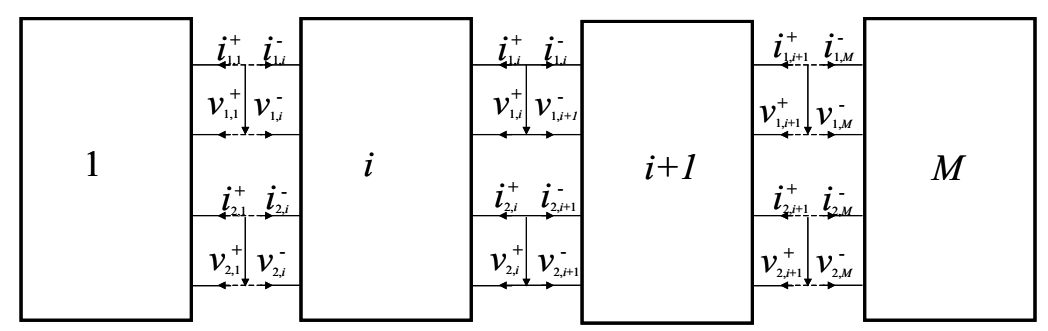

Figure 6.: Network Model

\subsubsection{Power Conserving Field Mapping}

As first step of the network determination, we describe the tangential components of the electric and magnetic fields to voltages and currents at the cylindrical interfaces. Using (64) we can directly map the cylindrical layer into a network. Referring to (64) one can directly observe that each inner layer must be described by a four port network for each mode. As discussed in section (6.1.3), the innermost and outermost layers are described by four fields quantities and therefore a two port network is used, Fig. 6. For non-TEM lines, a GTLM must be used. For implementation issues, it is advantageous that the network is power conservative. Therefore, an additional radius dependent normalization is introduced to achieve power conservation in the network model. In particular, in the lossless case, the power flowing through cylindrical surfaces containing the same dielectric material must be constant. Using the Poynting's vector and considering power per unit length (as it is reasonable for a structure of infinite extension) one can write

$$
\int_{\varphi=0}^{2 \pi} \operatorname{Re}\left\{\mathcal{E} \wedge \mathcal{H}^{*}\right\}\left(\rho_{i}^{-}\right) \cdot \mathbf{u}_{\rho} \rho_{i}^{-} d \varphi=\int_{\varphi=0}^{2 \pi} \operatorname{Re}\left\{\mathcal{E} \wedge \mathcal{H}^{*}\right)\left(\rho_{i}^{+}\right\} \cdot \mathbf{u}_{\rho} \rho_{i}^{+} d \varphi
$$

That simplifies to

$$
\left(\bar{E}_{\varphi, i}^{-}\left(\bar{H}_{z, i}^{-}\right)^{*}+\bar{E}_{z, i}^{-}\left(-\bar{H}_{\varphi, i}^{-}\right)^{*}\right) \rho_{i}^{-}=\left(\bar{E}_{\varphi, i}^{+}\left(\bar{H}_{z, i}^{+}\right)^{*}+\bar{E}_{z, i}^{+}\left(-\bar{H}_{\varphi, i}^{-}\right)^{*}\right) \rho_{i}^{+}
$$


From the (97) we can observe that to achieve a power conservative network the field amplitudes must be proportional to the $\sqrt{\rho_{i}^{ \pm}}$. We map the field in the following way:

$$
\begin{array}{ll}
\frac{v_{1, i}^{ \pm}}{v_{0}}=\frac{\bar{E}_{\varphi_{n}, i}^{ \pm}}{E_{0}} \frac{\sqrt{\rho_{i}^{ \pm}}}{\sqrt{\rho_{0}}} & \frac{v_{2, i}^{ \pm}}{v_{0}}=\frac{\bar{E}_{z_{n}, i}^{ \pm}}{E_{0}} \frac{\sqrt{\rho_{i}^{ \pm}}}{\sqrt{\rho_{0}}} \\
\frac{i_{1, i}^{ \pm}}{v_{0}}=\frac{\bar{H}_{z_{n}, i}^{ \pm}}{H_{0}} \frac{\sqrt{\rho_{i}^{ \pm}}}{\sqrt{\rho_{0}}} & \frac{i_{2, i}^{ \pm}}{i_{0}}=\frac{\bar{H}_{\varphi_{n}, i}^{ \pm}}{H_{0}} \frac{\sqrt{\rho_{i}^{ \pm}}}{\sqrt{\rho_{0}}}
\end{array}
$$

where $E_{0}, H_{0}$ and $\rho_{0}$ are arbitrarily chosen normalization constants ensuring appropriate units. With this field mapping, the electromagnetic boundary conditions at the interfaces (90) are translated into connection between the ports:

$$
\left(\begin{array}{c}
v_{1, i}^{+} \\
i_{1, i}^{+} \\
v_{2, i}^{+} \\
-i_{2, i}^{+}
\end{array}\right)=\left(\begin{array}{c}
v_{1, i+1}^{-} \\
i_{1, i+1}^{-} \\
v_{2, i+1}^{-} \\
-i_{2, i+1}^{-}
\end{array}\right) .
$$

With the field mapping as described above, this relation is equivalent to the condition for lossless networks of $\operatorname{Re}\left\{\mathbf{v}_{i} \mathbf{i}_{i}^{*}\right\}=0$ where $\mathbf{v}_{i}=\left(v_{1, i}^{-}, v_{2, i}^{-}, v_{1, i}^{+}, v_{2, i}^{+}\right)^{T}$ and $\mathbf{i}_{i}=\left(i_{1, i}^{-}, i_{2, i}^{-}, i_{1, i}^{+}, i_{2, i}^{+}\right)^{T}$. Therefore lossless cylindrical structures are mapped to lossless networks. The normalization factor $\sqrt{\rho}$ in the field mapping accounts for the radial dependence of the power density for cylindrical waves. Based on this and using (64) and (98) we obtain a parametric description

$$
\left(\begin{array}{c}
\mathbf{N}^{+} \\
\mathbf{N}^{-}
\end{array}\right)=\left(\begin{array}{c}
\mathbf{V}^{+} \\
\mathbf{I}^{-}
\end{array}\right)_{8 \times 4}\left(\begin{array}{c}
A_{n}^{i} \\
B_{n}^{i} \\
C_{n}^{i} \\
D_{n}^{i}
\end{array}\right)
$$

where $\mathbf{N}^{+}=\left(v_{1}^{i^{+}}, i_{1}^{i^{+}}, i_{2}^{i^{+}}, v_{2}^{i^{+}}\right)$and $\mathbf{N}^{+}=\left(v_{1}^{i^{-}}, i_{1}^{i^{-}}, i_{2}^{i^{-}}, v_{2}^{i^{-}}\right)$. Any other parameter, e.g. impedance $(Z)$, admittance $(Y)$ and also chain $(A)$ may be derived form (100). Alternative descriptions based on wave quantities can be calculated, in particular scattering $S$ and transmission $T$ matrix forms.

\section{Source modeling in the network description}

In such representation the sources are placed at the interfaces of the cylindrical layers. Using the network representation the interfaces are represented 


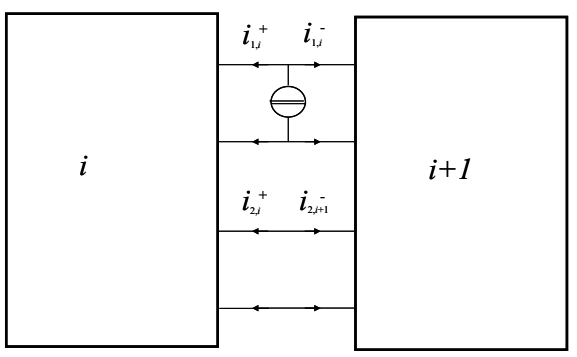

Figure 7.: Source in the Network Model

by connection between ports. Considering the (91), the discontinuity on the tangential component of the magnetic field will be translated into a step current discontinuity. In case of network it is relatively easy interpret the contribute of the Dirac source. In particular considering the (88) and that in case of Dirac source $J_{k_{z}}\left(k_{z}\right)=1$ and $J_{\varphi}(n)=1 / \pi$ for $n=0,1,2 \ldots$, the current $i_{1,2}$ can be easily defined by $i_{1,2}=\int_{\rho^{+}-\epsilon}^{\rho^{-}-\epsilon} \frac{J_{0}}{\pi} \delta\left(\rho-\rho_{i}\right) d \rho_{i}$. Defining $\frac{J_{0}}{\pi}=1$ the result is that the step discontinuity can be represented by a unitary generator of current as depicted in Fig. 7. Depending on the orientation of the sources, the generator is placed at port 1 or 2 or both. Once that the network has been excited, the amplitudes of the modal coefficients i.e. the amplitudes of voltage and current can be calculated in the entire network. We choose $E_{0}$ so that $v_{0} \sqrt{\rho_{0}} / E_{0}=1$ and from (201) and the (98) we obtain:

$$
\bar{G}_{p q}^{i j}\left(\rho, \rho^{\prime}, k_{z}, n\right)=\left.\bar{E}_{p_{n}}^{i}\left(\rho, n, k_{z}\right)\right|_{\mathbf{J}(\mathbf{r})=\mathbf{u}_{q} \delta\left(\mathbf{r}-\mathbf{r}^{\prime}\right)}=\frac{v_{1,2}^{i}}{\sqrt{\rho_{i}^{ \pm}}}
$$

where $\bar{G}_{p q}^{i j}\left(\rho, \rho^{\prime}, k_{z}, n\right)$ are the spectral domain components of the p-directed Green's function due to the q-oriented source. Finally, the space domain Green's functions are given by:

$$
G_{p q}^{i j}\left(\mathbf{r}, \mathbf{r}^{\prime}\right)=\frac{j}{8 \pi} \int_{k_{z}} \sum_{n=0}^{\infty} d k_{z} \bar{G}_{p q}^{i j}\left(\rho, \rho^{\prime}, k_{z}, n\right) e^{-j n\left(\varphi-\varphi^{\prime}\right)} e^{-j k_{z}\left(z-z^{\prime}\right)}
$$




\subsection{Space Domain Green's functions}

Once that spectral domain Green's functions are obtained by using the GTLM spatial domain Green's functions must be calculated. The transformation contains a classical Fourier type integral given in (102). Such kind of integral cannot be solved analytically. On the other hands, the use of standard numerical integration techniques (e.g. trapezoidal) may be very problematic due to the characteristics of the integral. Problems arise from the facts, that the integration range is infinitely extended, the integrand may contain rapidly oscillating terms and slowly decaying exponential terms. For fast oscillating functions a high density sampling rate would be necessary, while for slowly decaying functions raise the need for an extended integration range. The combination of this two conditions results in a huge number of samples and consequently long simulation time is required. The advantages of the analytical pre-calculation of the Green's function are therefore not effective. An other important consequence is robustness. Using a merely numerical technique, the characteristic of the integrand function i.e. oscillating frequency and decay rate, must be analyzed a-priori. In fact the wrong choice of the integration parameters produces not correct results [16]. Therefore, a more advanced integration technique based on the Generalized Pencil of Function (GPOF) [17,6, 15, 16] will be used. Based on this approach an alternative representation of the Green's function will introduced in Section 6.3.1. In solving the Fourier integral (102), further problems arise due to the singularities. This problem will be treated in the next section.

\subsubsection{Analysis of the singularities}

The kernel of the Fourier internal (102) contains the radial spectral domain Green's functions $\bar{G}_{p q}^{i j}\left(\rho, \rho^{\prime}, k_{z}, n\right)$ given in (101). This is a function of the radial wavenumber $k_{\rho_{i}}$ for all the cylindrical layers

$$
\bar{G}_{p q}^{i j}\left(\rho, \rho^{\prime}, k_{z}, n\right)=T_{k l}\left\{\begin{array}{l}
A_{n}^{i} H_{n}^{(1)}\left(k_{\rho_{i}} \rho\right)+B_{n}^{i} H_{n}^{(2)}\left(k_{\rho_{i}} \rho\right) \\
C_{n}^{i} H_{n}^{(1)}\left(k_{\rho_{i}} \rho\right)+D_{n}^{i} H_{n}^{(2)}\left(k_{\rho_{i}} \rho\right)
\end{array}\right.
$$


where $T_{k l}$ is an element of the linear operator $\mathbf{T}$ defined as follows

$$
\mathbf{T}=\left(\begin{array}{cc}
\frac{k_{\rho_{i}}^{2}}{j \omega \epsilon_{i}}(\bullet) & -\frac{\partial}{\partial \rho}(\bullet)+\frac{j n k_{z}}{j \omega \mu_{i} \rho}(\bullet) \\
\frac{k_{\rho_{i}}^{2}}{j \omega \mu_{i}}(\bullet) & \frac{j k_{z} n}{j \omega \epsilon_{i} \rho}(\bullet)+\frac{\partial}{\partial \rho}(\bullet)
\end{array}\right)_{<2 \times 2>}
$$

For each layer $k_{\rho_{i}}=\sqrt{k_{i}^{2}-k_{z}^{2}}$, where $k_{i}$ is the wavenumber of the $i$ th layer and $k_{z}$ is the axial wavenumber which have to be the same for all the layers by the phase matching. The Hankel functions are singular functions for $k_{\rho_{i}} \rho \rightarrow 0$. In the lossless case $k_{\rho_{i}}=0$ when $k_{z}= \pm k_{i}$, Figure 8. Because of $k_{\rho_{i}}$ is a multivalue function, e.g. Figure 9 , the singularities may be poles or branch points [27].

The complex values at $k_{z}= \pm k_{i}$ are not branch points [26] in the layers $i=1,2, . ., M-1$ due to the fact the regions are limited in $\rho$. In particular, considering that any two independent solution of the wave equation are solution of the wave equation (59), with a different choice of different branches of the Hankel functions they are still solution of the same wave equation and the same boundary conditions are satisfied. Therefore, considering the uniqueness theorem, if the boundary conditions are unchanged, the same field quantities are obtained for any arbitrary choice of the branch and consequently they are single value functions.

We consider now the region unbounded in $\rho$ and the source embedded in the layer $1 \leq j<M$. The unbounded region corresponds with the layer $M$ in which the modal coefficients $A_{n}^{M}=C_{n}^{M}=0$. Therefore the spectral domain Green's function (103) is characterized by Hankel function of the second kind

$$
\bar{G}_{p q}^{M j}\left(\rho, \rho^{\prime}, k_{z}, n\right)=T_{k l}\left\{\begin{array}{l}
B_{n}^{M} H_{n}^{(2)}\left(k_{\rho_{M}} \rho\right) \\
D_{n}^{M} H_{n}^{(2)}\left(k_{\rho_{M}} \rho\right)
\end{array}\right.
$$

In this case the branch cut cannot be chosen arbitrarily since the radiation condition must be satisfied when $\rho \rightarrow+\infty$. In order to identify the correct branch and classify the kind of singularities we consider the following approximation of the Hankel function of the second kind when $\rho \rightarrow+\infty$

$$
H_{n}^{(2)}\left(k_{\rho_{M}} \rho\right) \backsim \sqrt{\frac{2}{\pi k_{\rho_{M}} \rho}} e^{-j\left(k_{\rho_{M}} \rho+(2 n+1) \pi / 4\right)} \quad n=0,1, \ldots,+\infty
$$




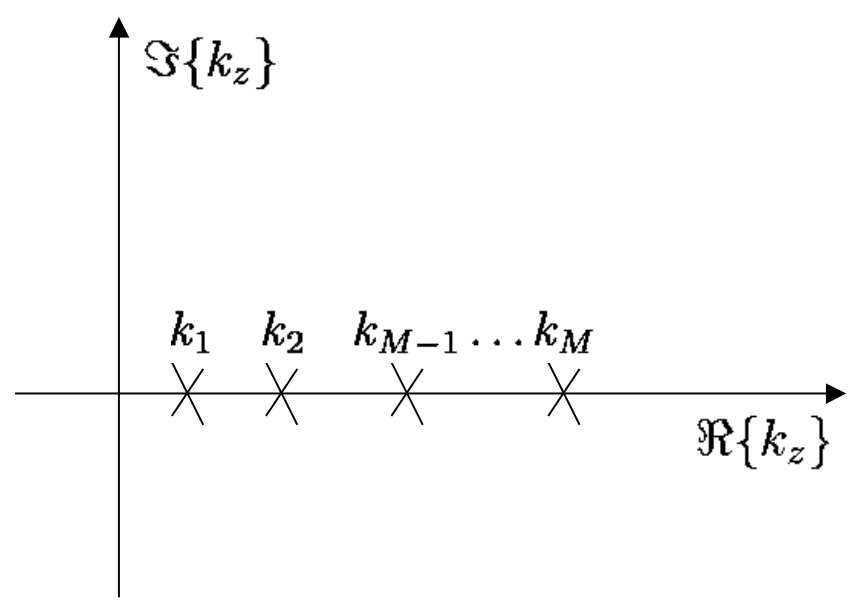

Figure 8.: Singularities in the complex plane $k_{z}$ in the lossless case.

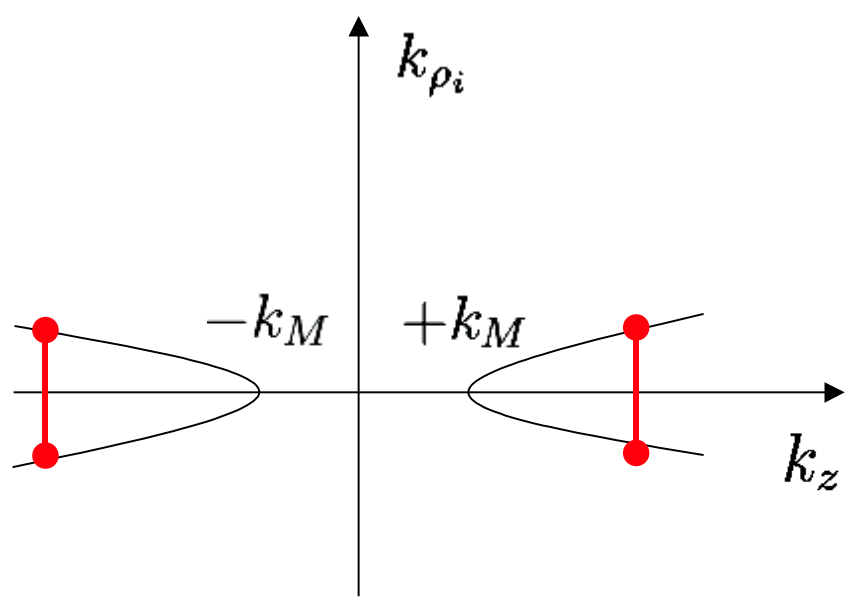

Figure 9.: In this example $k_{\rho_{i}}$ is real. 


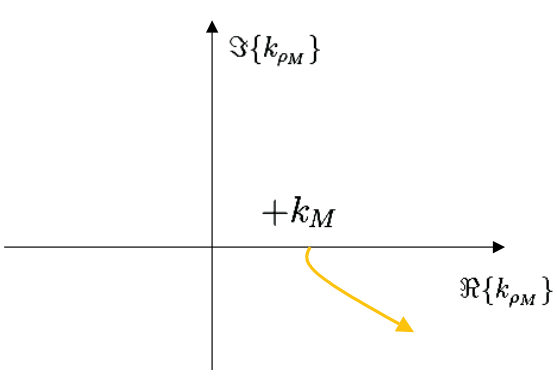

(i) Principal branch.

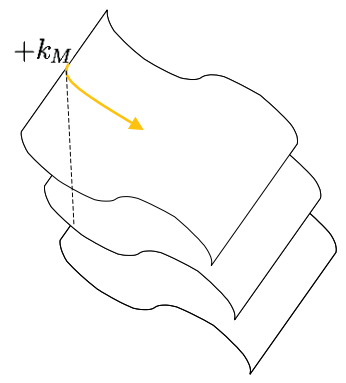

(ii) The Riemann sheets.

Figure 10.: Qualitative view of the principal branch and Reimann sheets.

We can rearrange (106) by separating the the real and imaginary part of $k_{\rho_{M}}$ as follows

$$
\sqrt{\frac{2}{\pi k_{\rho_{M}} \rho}} e^{-j\left(k_{\rho_{M}} \rho+(2 n+1) \pi / 4\right)}=\sqrt{\frac{2}{\pi k_{\rho_{M}} \rho}} e^{(2 n+1) \pi / 4)} e^{\lambda_{i} \rho} e^{-j \lambda_{r} \rho}
$$

where $\lambda_{r}=\Re\left\{k_{\rho_{M}}\right\}$ and $\lambda_{i}=\Im\left\{k_{\rho_{M}}\right\}$. Considering (107), in the layer $M$ only outward $(+\rho)$ cylindrical waves exist and consequently the $\lambda_{r} \geqslant 0$. Moreover, to satisfy the radiation condition $\lambda_{i} \leqslant 0$ since for $\rho \rightarrow+\infty$ must be that $e^{\lambda_{i} \rho} \rightarrow 0$. Therefore only the branch in the 4 th quadrant is allowed Figure 10i. We may conclude that the $\pm k_{M}$ are singularities branch points. The other singularities are poles and represent the guided and unguided modes in the layered region and my be classified as leaky, creeping or trapped waves [26,32] and can be found in all the Riemann sheets corresponding to the branch point of the outer layer, Figure 10ii. The poles which are not in other Riemann sheets are modes which not satisfy the radiation condition.

\subsubsection{Choice of the Contour of Integration}

As mentioned before, in the lossless case the singularities are on the real axis at $k_{z}= \pm k_{i}$. Since the integral is not defined through singularities 
poles and branch points, the integration contour cannot be chosen to be the real axis. Therefore an alternative integration contour must be found.

Considering the Cauchy's theorem [34] the integral in the complex plane between two points is invariant for any arbitrary integration contour not passing singularities. Therefore we are allowable to replace the (102) as follows

$$
G_{p q}^{i j}\left(\mathbf{r}, \mathbf{r}^{\prime}\right)=\frac{j}{8 \pi} \sum_{n=0}^{\infty} e^{-j n\left(\varphi-\varphi^{\prime}\right)} \int_{\Gamma} \bar{G}_{p q}^{i j}\left(\rho, \rho^{\prime}, k_{z}, n\right) e^{-j k_{z}\left(z-z^{\prime}\right)} \mathrm{d} k_{z}
$$

where $\Gamma$ is the new integration contour of the complex plane $\mathbb{C}$. On the other hands, since, by the Poincaré-Volterra Lemma [34], any analytic function posses a countably many distinct values at any point of the complex plane $\mathbb{C}$, the analytic functions are always multivalue. That means that we have to choose a region of the complex $\Gamma$-plane in which the integrand function is single value. As discussed in the previously for the complex $k_{\rho}$-plane, if we choose the branch which passes for the 4 th quadrant, the radiation condition is satisfied and together with the boundary conditions the uniqueness is guarantied. Accordingly, we may choose the integration contour in the complex $k_{z}$-plane. In particular, it must be that $\Re\left\{k_{z}\right\} \geq 0$ and $\Im\left\{k_{z}\right\} \geq 0$ which is easily recognize to be the 4 th quadrant in the complex $k_{\rho}$-plane.

As next steep we define complex contour $\Gamma$. Here some considerations should be made. Since we perform the integral numerically, the choice of the shape of $\Gamma$ is related with the selection of the integration technique. The numerical evaluation of the integral is a sensitive problem due to the discretization of the wavenumber and the infinite integration range. The first one gives rise to aliasing and wrap-around errors because of the oscillatory nature of the Hankel functions. The second aspect, related with the integration range is even more severe. The Cauchy's Theorem of Residues guaranties in the continuous analytical cases that the integral is the summation over all the residues of the function choosing an integration contour enclosing all the singularities. If as in this case, the number of poles-singularities may be found in an infinite range of values given by $k_{z}$ then the contour of integration is extended to infinite. On the other hands, numerically the integration range must be truncated. In this case some significant poles of the integrand function, may be not included and therefore an a preliminary in- 
vestigation of the behavior of the integrand function must be performed [16]. If the number of the cylindrical layer $M$ is relatively large, e.g. 3-4, then the complexity of the $\bar{G}_{p q}^{i j}\left(\rho, \rho^{\prime}, k_{z}, n\right)$ increases while a pre-analysis of its behavior becomes very complex.

Therefore we select an alternative integration technique based on Prony's method [36] introduced by Chow et al. [35]. The method enables to expand $\bar{G}_{p q}^{i j}\left(\rho, \rho^{\prime}, k_{z}, n\right)$ into a series of exponential functions in which the amplitude of each exponential function is the value of the residue and its argument represent the estimated position of the pole. The method is used in connection with a Mean Square Error(MSE) criterium introduced by [23]. The MSE criteria allows a self-consistent estimation of the estimation error of the poles and therefore of the truncation point. Further details on the Prony's method will be given in Section 6.4. In the following we will define the discretization of the wavenumber discretization of the integration contour.

\section{Definition of $\Gamma$ (Complex Integration Contur $)$}

In the choice of $\Gamma$ we must consider that any complex function of a real variable of a finite range can be approximated by the summation of exponential functions using the Prony's method [35]. The choice of the classical Prony's method requires uniform sampling of the complex valued function. Therefore we split the contours in three linear Sections $\Gamma_{1}, \Gamma_{2}$ and $\Gamma_{3}$, Figure 11. The segmentation of the integration path in three parts brings some important numerical advantages that will be explained later. The segmentation

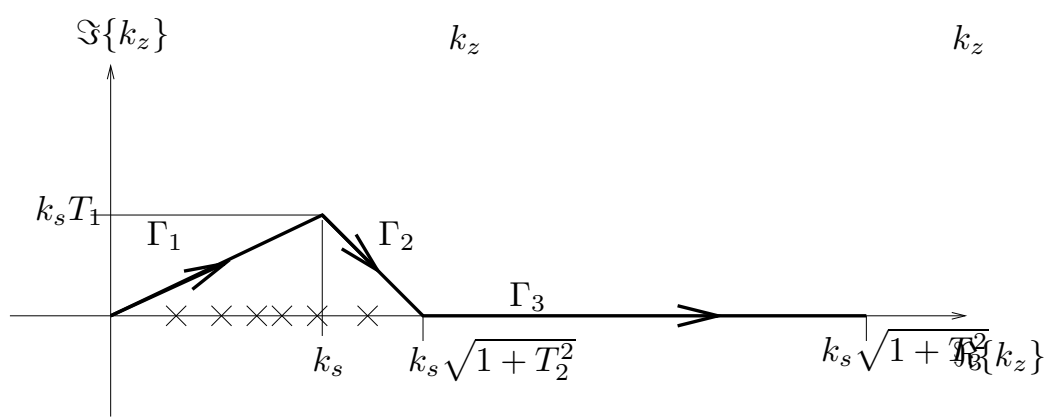

Figure 11.: Integration Path for Integration of Spectral Domain Green's Function, Path Parameters 


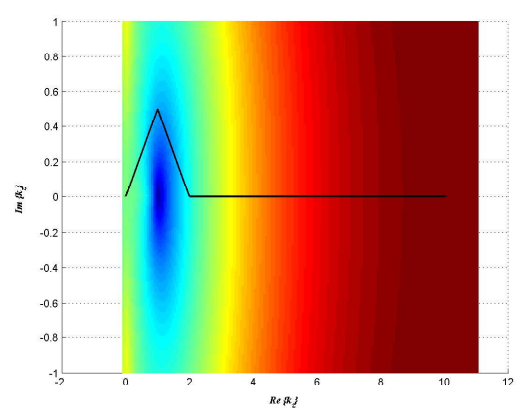

(i)

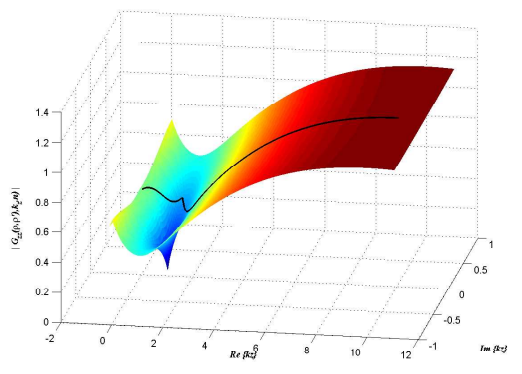

(iii)

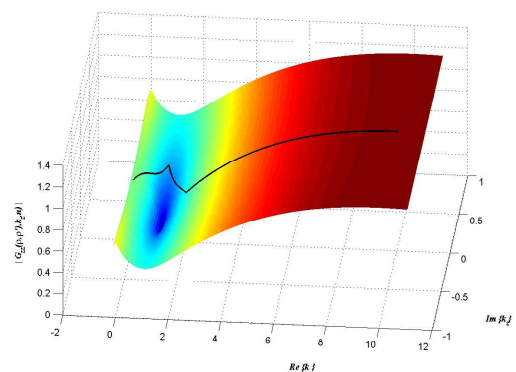

(ii)

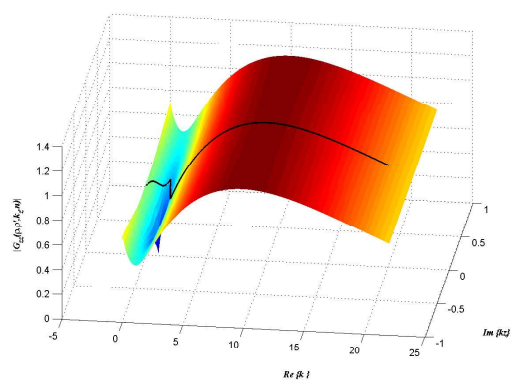

(iv)

Figure 12.: Visualization of the integration path with the $\left|\bar{G}_{z z}\left(\rho, \rho^{\prime}, k_{z}, n\right)\right|$ in free space at frequency of $0.8 \mathrm{GHz}$.

of the integration path can be represented by the parametric equation of a straight line between two points $\left(\lambda_{z_{r}}^{(1)}, \lambda_{z_{i}}^{(1)}\right)$ and $\left(\lambda_{z_{r}}^{(2)}, \lambda_{z_{i}}^{(2)}\right)$ of the complex plane $k_{z}$ as follows

$$
\begin{aligned}
& \lambda_{z_{r}}(t)=\lambda_{z_{r}}^{(1)}+\left(\lambda_{z_{r}}^{(2)}-\lambda_{z_{r}}^{(1)}\right) t \\
& \lambda_{z_{i}}(t)=\lambda_{z_{i}}^{(1)}+\left(\lambda_{z_{i}}^{(2)}-\lambda_{z_{i}}^{(1)}\right) t
\end{aligned}
$$

where $t$ is a real number in the interval $[0,1], \lambda_{z_{r}}^{(1,2)}=\Re\left\{k_{z}\right\}$ and $\lambda_{z_{i}}^{(1,2)}=$ $\Im\left\{k_{z}\right\}$. It easy to see that for $\Gamma_{1},\left(\lambda_{z_{r}}^{(1)}, \lambda_{z_{i}}^{(1)}\right)=(0,0)$ and $\left(\lambda_{z_{r}}^{(2)}, \lambda_{z_{i}}^{(2)}\right)=$ $\left(k_{s}, k_{s} T_{1}\right)$, for $\Gamma_{2},\left(\lambda_{z_{r}}^{(1)}, \lambda_{z_{i}}^{(1)}\right)=\left(k_{s}, k_{s} \sqrt{1+T_{2}^{2}}\right)$ and $\left(\lambda_{z_{r}}^{(2)}, \lambda_{z_{i}}^{(2)}\right)=\left(k_{s}\right.$ 
$\left.\sqrt{1+T_{2}^{2}}, 0\right)$ and for $\Gamma_{3}\left(\lambda_{z_{r}}^{(1)}, \lambda_{z_{i}}^{(1)}\right)=\left(k_{s} \sqrt{1+T_{2}^{2}}, 0\right),\left(\lambda_{z_{r}}^{(2)}, \lambda_{z_{i}}^{(2)}\right)=\left(k_{s}\right.$ $\sqrt{1+T_{3}^{2}}, 0$ ), Figure 11. We observe that transformation( 109) and (110) map the real variable $t$ into a complex plane $k_{z}$ and therefore the GPOF can be used. In the definition of the points $\left(x_{i}, y_{i}\right)$ with $(i=1,2)$ we have introduced the parameters $k_{s}, T_{1}, T_{2}$ and $T_{3}$.

The choice of the integration parameters is related to the Cauchy's theorem and the numerical integration technique by considerations made before. In fact truncation of the integration interval at $k_{z_{\max }}$ is defined by $k_{s} \sqrt{1+T_{3}^{2}}$ which for completely fulfilling the Cauchy's theorem should be extended at infinite in order to close the integration path. If the integration interval is truncated then the effect of the $\Im\left\{k_{z}\right\}$ which is defined by $k_{s} T_{1}$ becomes relevant. If $k_{s} T_{1} \ll k_{z_{\max }}$ and $\bar{G}_{p q}^{i j}\left(\rho, \rho^{\prime}, k_{z_{\max }}, n\right) \simeq 0$, then the effect from the vertical sections $\Gamma_{1}$ and $\Gamma_{2}$ become insignificant compared to the integral along the horizontal section defined by $\Gamma_{3}$, see Figure 6.3.2. Finally the choice of $T_{2}$ such that $k_{s} \sqrt{1+T_{2}^{2}}>\max \left\{k_{i}\right\}$ insures that integration path does not pass through branch points and singularities.

\section{Discretization of the wavenumber}

To numerically compute the integral (102) the integration kernel $\bar{G}_{p q}^{i j}\left(\rho, \rho^{\prime}\right.$, $\left.k_{z}, n\right)$ must be evaluated at discrete number of wavenumbers. As it was mentioned, the classical GPOF method requires a uniform sampling of the kernel function, and therefore we discretize the truncated wavenumber space equidistantly as follows

$$
\begin{array}{r}
\lambda_{z_{r}}(t)=\lambda_{z_{r}}^{(1)}+\Delta \lambda_{z_{r}} T(l-1), \\
\lambda_{z_{i}}(t)=\lambda_{z_{i}}^{(1)}+\Delta \lambda_{z_{i}} T(l-1) . \\
t=T(l-1) \\
T=\frac{1}{N-1} \\
l=1,2, \ldots, N
\end{array}
$$

where $\Delta \lambda_{z_{r, i}}=\left(\lambda_{z_{r}}^{(2)}-\lambda_{z_{r}}^{(1)}\right)$ and $k_{z}(t)=k_{z}=\lambda_{z_{r}}(t)+j \lambda_{z_{i}}(t)$. In order to simplify the notation we redefine the spectral domain Green's function in the following way

$$
\bar{G}_{p q}^{i j}\left(\rho, \rho^{\prime}, k_{z}, n\right)=\bar{G}_{n}\left(k_{z}\right)
$$


The Green's function is then sampled along the chosen integration path and approximated in terms of poles expansion model as follows

$$
\bar{G}_{n}\left(k_{z}\right) \approx \bar{G}_{n}(l)=\sum_{p=1}^{N_{e}} b_{p}\left(z_{p}\right)^{l-1} .
$$

where $N_{e}$ is the estimated number of poles, $z_{p}^{n}$ and $b_{p}^{n}$ are poles and residues respectively [16]. The parametric model of the Green's function given in (113) is usually called expansion in natural frequencies. The natural frequencies are given by the complex quantities $z_{p}=e^{s_{p} T}$, where $T$ is the discretization interval and in this case $s_{p}$ are cylindrical eigenvalues. The technique here used for estimating the poles and residues will be discussed in more details in Section 6.4. We proceed inserting the $z_{p}=e^{s_{p} T}$ and $l-1=t / T$ into (113) we obtain the following parametric representation of the Green's function

$$
\bar{G}_{n}(t) \approx \sum_{p=1}^{N_{e}} b_{p}^{n} e^{s_{p}^{n} t} .
$$

Next we insert (112) into (108) we obtain

$$
G_{p q}^{i j}\left(\mathbf{r}, \mathbf{r}^{\prime}\right)=\frac{j}{8 \pi} \sum_{n=0}^{\infty} e^{-j n\left(\varphi-\varphi^{\prime}\right)} \int_{\Gamma} \bar{G}_{n}\left(k_{z}\right) e^{-j k_{z}\left(z-z^{\prime}\right)} \mathrm{d} k_{z}
$$

To simplify the notation we define

$$
I_{\Gamma_{n}}=\int_{\Gamma} \bar{G}_{n}\left(k_{z}\right) e^{-j k_{z}\left(z-z^{\prime}\right)} \mathrm{d} k_{z}
$$

which must be solved for every $n$. Inserting (111) into (116), considering the property of the parametric integral of a complex function, see Appendix B, we obtain

$$
I_{\Gamma_{n}}=\left(\Delta \lambda_{z_{r}}+j \Delta \lambda_{z_{i}}\right) \int_{t=0}^{1} \bar{G}_{n}(t) e^{-j\left(z-z^{\prime}\right)\left(\lambda_{z_{r}}^{(1)}+j \lambda_{z_{i}}^{(1)}+\left(\Delta \lambda_{z_{r}}+j \Delta \lambda_{z_{i}}\right) t\right)} \mathrm{d} t
$$


Defining $\Delta h=\left(z-z^{\prime}\right)\left(\Delta \lambda_{z_{r}}+j \Delta \lambda_{z_{i}}\right)$ and $h=\left(z-z^{\prime}\right)\left(\lambda_{z_{r}}+j \lambda_{z_{i}}\right)$ we can rewrite (117) as follows

$$
\begin{array}{r}
I_{\Gamma_{n}}=g \int_{\Gamma} \bar{G}_{n}(t) e^{-j \Delta h t} \mathrm{~d} t \\
g=\frac{\Delta h e^{-j h}}{\left(z-z^{\prime}\right)}
\end{array}
$$

Using (114) into (118) we obtain

$$
I_{\Gamma_{n}}=g \sum_{p=1}^{N_{e}} \int_{0}^{1} b_{p}^{n} e^{s_{p}^{n} t} e^{-j \Delta h t} \mathrm{~d} t=g \sum_{p=1}^{N_{e}} b_{p}^{n} \frac{e^{\left(s_{p}^{n}-j \Delta h\right)}-1}{s_{p}^{n}-j \Delta h}
$$

Substituting (119) into (115) we obtain the space Green's function expression as follows

$$
G_{p q}^{i j}\left(\mathbf{r}, \mathbf{r}^{\prime}\right)=\frac{j g}{8 \pi} \sum_{n=0}^{\infty} \sum_{p=1}^{N_{e}} b_{p}^{n} \frac{e^{\left(s_{p}^{n}-j \Delta h\right)}-1}{s_{p}^{n}-j \Delta h} e^{-j n\left(\varphi-\varphi^{\prime}\right)}
$$

We now make some further considerations on the solution. The (119) represents the results of the Fourier integral for the semi-complex $k_{z}$-plane extended form 0 to $+\infty$. The complete solution is completely solution. Since we have assumed the multilayered cylindrical structure infinitively extended, the solution in the semi-complex $k_{z}$-plane extended form 0 to $-\infty$ can be can be considered completely symmetric. Therefore the complete solution in space domain is given as follows

$$
G_{p q}^{i j}\left(\mathbf{r}, \mathbf{r}^{\prime}\right)=\frac{j g}{8 \pi} \sum_{n=0}^{\infty} \sum_{p=1}^{N_{e}} b_{p}^{n}\left[\frac{e^{\left(s_{p}^{n}-j \Delta h\right)}-1}{s_{p}^{n}-j \Delta h}+\frac{e^{\left(s_{p}^{n}+j \Delta h\right)}-1}{s_{p}^{n}+j \Delta h}\right] e^{-j n\left(\varphi-\varphi^{\prime}\right)}
$$

\subsection{The Generalized Pencil of Function(GPOF) Algorithm}

The (113) represents a parametric model description of the spectral domain Green's function in terms of space poles $s_{p}^{n}$ and residues $b_{p}^{n}$. The approximation is performed over a summation of $N_{e}$ complex exponential terms 
which represent the model order. Therefore the parametric approximation of the Green's function requires the knowledge of the model parameters $s_{p}^{n}$, $b_{p}^{n}$ and $N_{e}$ to be completely characterized. The System Identification(SI) theory offers a number of techniques for the estimation of the model parameters. In the present algorithm the SVD will be used for the order estimation, the generalized PM based method, for the poles identification and the LS approximation is used for computing the residues $b_{p}^{n}$ by model fitting to the available data. In the following Sections a brief review of the GPOF algorithm will be given.

\subsubsection{Model Order Selection}

As first step the number of exponential functions/or the number of poles $N_{e}$ is estimated. The ensemble of Green's function available samples can be rearranged in a vector a vector of length $N, \mathbf{G}_{p}=[G[0] G[1] \ldots G[N-1]]^{T}$. The (113) can be rewritten in matrix form as follows:

$$
\mathbf{G}_{p} \simeq \hat{\mathbf{G}}=\mathbf{Z} \mathbf{b} .
$$

where $\mathbf{Z}$ is the poles based Vandermonde matrix given by

$$
\mathbf{Z}=\left[\begin{array}{cccc}
1 & 1 & \cdots & 1 \\
z_{1} & z_{2} & \cdots & z_{N_{e}} \\
\vdots & \vdots & & \vdots \\
z_{1}^{N-1} & z_{2}^{N-1} & \cdots & z_{N_{e}}^{N-1}
\end{array}\right]
$$

and where $z_{i}, i=1,2, \ldots, N_{e}$ are the poles, $\mathbf{b}=\left[b_{1}, b_{2}, \ldots, b_{N_{e}}\right]^{T}$ the residues vector. The model order $N_{e}$, can be estimated by using the SVD of the correlation matrix $\mathbf{R}$. The correlation matrix is an $(L+1) \times(L+1)$ matrix given as follows

$$
\mathbf{R}=\mathbf{G}^{H} \mathbf{G}
$$

where $L$ is an integer number called also pencil parameter. The choice of $L$ is a sensitive to the data noise reduction. It has been shown in literature that for an efficient noise filtering the pencil parameter gives minimum noise effects within the range $[N / 3, N / 2][17]$. After experimental testing it turned 
out that in our implementation, the best noise behavior is given for $L=$ $\left\lfloor\frac{N}{2}\right\rfloor$. The $\mathbf{G}$ is a $(N-L) \times(L+1)$ data matrix given by

$$
\mathbf{G}=\left[\begin{array}{llll}
\mathbf{G}_{L} & \mathbf{G}_{L-1} & \ldots & \mathbf{G}_{0}
\end{array}\right]
$$

with $\mathbf{G}_{k}$ being an $(N-L) \times 1$ data vector given as

$$
\mathbf{G}_{k}=[G[k] G[k+1] \ldots G[N-L+k-1]]^{T}
$$

and $\mathbf{G}^{H}$ represents the Hermitian matrix of $\mathbf{G}$. Using the SVD, the matrix $\mathbf{R}$ can be written as follows

$$
\mathbf{R}=\mathbf{U} \boldsymbol{\Sigma} \mathbf{V}
$$

where the $\mathbf{U}$ and $\mathbf{V}$ are the matrices made of the unitary eigenvectors of $\mathbf{R R}^{H}$ and $\mathbf{R}^{H} \mathbf{R}$, and $\boldsymbol{\Sigma}$ is the diagonal matrix given as $\boldsymbol{\Sigma}=\operatorname{diag}\left\{\sigma_{0}, \sigma_{1}\right.$, $\left.\ldots, \sigma_{r},\right\}$, with the positive quantities $\sigma_{0} \geq \sigma_{1} \geq \ldots \sigma_{r} \geq 0$ singular values of $\mathbf{R}$. Since in signal corrupted by noise the singular values correspondent to the signal component are dominating those correspondent to the noise [23], the model order or signal subspace dimension can be found by selecting the $P$ bigger singular values. In order to do this a threshold value $D_{0}$ is fixed and the number $P$ is chosen such that the singular values SNR defined as

$$
\operatorname{SNR}\left(N_{e}\right)=10 \log _{10} \frac{\sum_{i=1}^{P} \sigma_{i}{ }^{2}}{\sum_{i=P+1}^{r} \sigma_{i}{ }^{2}}
$$

satisfies the relation

$$
\operatorname{SNR}\left(N_{e}\right) \geq D_{0}
$$

\subsubsection{Poles estimation by GPOF method}

Analogous to the Pencil Method(PM) [37] we collect the data sample information of the spectral domain Green's functions in two data matrixes $\mathbf{G}_{0}$ and $\mathbf{G}_{1}$ of dimensions $(N-L) \times L$ given by

$$
\begin{aligned}
\mathbf{G}_{0} & =\left[\begin{array}{llll}
\mathbf{G}_{L-1} & \mathbf{G}_{L-2} & \ldots & \mathbf{G}_{0}
\end{array}\right] \\
\mathbf{G}_{1} & =\left[\begin{array}{llll}
\mathbf{G}_{L} & \mathbf{G}_{L-1} & \ldots & \mathbf{G}_{1}
\end{array}\right]
\end{aligned}
$$


Following the PM idea, looking the matrix structures of (130) we can rewrite them as follows [16,23]

$$
\begin{aligned}
\mathbf{G}_{0} & =\mathbf{Z}_{1} \mathbf{B} \mathbf{Z}_{2} \\
\mathbf{G}_{1} & =\mathbf{Z}_{1} \mathbf{B} \mathbf{Z}_{0} \mathbf{Z}_{1}
\end{aligned}
$$

where the matrix $\mathbf{Z}_{1,2}$ are given as follows:

$$
\begin{gathered}
\mathbf{Z}_{1}=\left[\begin{array}{cccc}
1 & 1 & \cdots & 1 \\
z_{1} & z_{2} & \cdots & z_{N_{e}} \\
\vdots & \vdots & & \vdots \\
z_{1}^{N-L-1} & z_{2}^{N-L-1} & \cdots & z_{N_{e}}^{N-L-1}
\end{array}\right] \\
\mathbf{Z}_{2}=\left[\begin{array}{cccc}
1 & z_{1} & \cdots & z_{1}^{L-1} \\
1 & z_{N_{e}} & \cdots & z_{N_{e}}^{L-1}
\end{array}\right]
\end{gathered}
$$

and $\mathbf{B}=\operatorname{diag}\left[b_{1}^{n}, b_{1}^{n}, \ldots, b_{N_{e}}^{n}\right], \mathbf{Z}_{0}=\operatorname{diag}\left[z_{1}^{n}, z_{1}^{n}, \ldots, z_{N_{e}}^{n}\right]$. Since the $\mathbf{G}_{0}$ and $\mathbf{G}_{1}$ are generally not full-rank [23] and not square $\left(N_{e} \leq L \leq N-N_{e}\right)$, the parameters $z_{i}^{n}$ with $i=1, \ldots, N_{e}$, are the generalized eigenvalues [38] of the of the following non-square pencil matrix

$$
\mathbf{P}(z)=\mathbf{G}_{0}-z_{i}^{n} \mathbf{G}_{1}
$$

Compared with standard Prony method [36] and the standard pencil of function method the poles are found directly solving the generalized eigenvalue problem given in (136). We now illustrate the algorithm for computing the generalized eigenvalues of (136). Considering (132) and (133) we can write

$$
\mathbf{G}_{0}^{\text {pi }} \mathbf{G}_{1}=\mathbf{Z}_{2}^{\text {pi }} \mathbf{Z}_{0} \mathbf{Z}_{2}
$$

where pi denotes the Moore-Penrose pseudo-inverse [38]. From (137), we can see the there are vectors $\mathbf{p}_{i}$ of $i=1, \ldots, N_{e}$ such that satisfy the following equations

$$
\begin{aligned}
\mathbf{G}_{0}^{\mathrm{pi}} \mathbf{G}_{0} \mathbf{p}_{i} & =\mathbf{p}_{i} \\
\mathbf{G}_{0}^{\mathrm{pi}} \mathbf{G}_{1} \mathbf{p}_{i} & =z_{i} \mathbf{p}_{i}
\end{aligned}
$$

where $\mathbf{p}_{i}$ are also called the generalized eigenvectors of the pencil matrix $\mathbf{P}(z)$. Next from the generalized eigenvectors we derive the generalized 
eigenvalues. The first step is the computation of the pseudo-inverse. The method, based on SVD, is extensively described in literature and here only the result will be given [38]

$$
\mathbf{G}_{0}^{\mathbf{p i}}=\mathbf{V D}^{-1} \mathbf{H}^{H}
$$

where $\mathbf{V}$ and $\mathbf{U}$ are the matrixes of the left and right singular vectors and $\mathbf{D}=\operatorname{diag}\left\{\sigma_{1}, \ldots, \sigma_{N_{e}}\right\}$ with $\sigma_{i}$ singular values. Considering (140), $\mathbf{G}_{0}^{\mathbf{p i}} \mathbf{G}_{0}=\mathbf{V} \mathbf{V}^{H}$ and $\mathbf{V}^{H} \mathbf{V}=\mathbf{I}$. inserting (140) into (139)

$$
\mathbf{V D}^{-1} \mathbf{H}^{H} \mathbf{G}_{1} \mathbf{p}_{i}=z_{i} \mathbf{p}_{i}
$$

left multiplying for $\mathbf{V}^{H}$

$$
\mathbf{V}^{H} \mathbf{V D}^{-1} \mathbf{H}^{H} \mathbf{G}_{1} \mathbf{p}_{i}=\mathbf{D}^{-1} \mathbf{H}^{H} \mathbf{G}_{1} \mathbf{p}_{i}=\mathbf{V}^{H} z_{i} \mathbf{p}_{i}
$$

Naming as $\mathbf{Z}_{\mathbf{p}}=\mathbf{D}^{-1} \mathbf{H}^{H} \mathbf{G}_{1} \mathbf{p}_{i}$ and $\mathbf{z}_{i}=\mathbf{V}^{H} \mathbf{p}_{i}$ and rearranging we obtain (142)

$$
\left(\mathbf{Z}-z_{i}^{n} \mathbf{I}\right) \mathbf{z}_{i}=0
$$

which is an equivalent eigenvalue problem to (136). In this case the matrix $\mathbf{Z}_{e}$ is a square matrix of dimensions $N_{e} \times N_{e}$ and therefore the eigenvalues $z_{i}^{n}$ can be calculated in the usual way as follows

$$
\operatorname{det}\left(\mathbf{Z}_{-} z_{i}^{n} \mathbf{I}\right)=0
$$

Knowing the generalized eigenvalues $z_{i}^{n}$ the value of the poles can be directly extracted in the following way

$$
s_{i}^{n}=\ln \left(z_{i}^{n}\right) / T
$$

To complete the description of the GPOF algorithm, furthers considerations can be made on the choice of pencil parameter $L$. Although the Prony method and GPOF come from two different principles, if $L=N_{e}$ the GPOF, the $\mathbf{G}_{0}$ is square and the residue inverse is not required and therefore the GPOF is equivalent to the Least Square (LS) Prony method to the original PM [17]. 


\subsubsection{LS Model fitting}

Once that the number of poles $N_{e}$ and the eigenvalues $z_{i}^{n}$ are known, the residues $b_{i}^{n}$ can be computed solving LS problems (122). This yields:

$$
\mathbf{b}=\left(\mathbf{Z}^{H} \mathbf{Z}\right)^{-1} \mathbf{Z}^{H} \mathbf{G}_{p}
$$

with $\mathbf{Z}$ defined as in (123). 


\section{Validation of the GPOF and Space Domain Green's Functions}

Key points in method developed in this work in computing the cylindrical Green's functions, are the correctness of the GPOF approximation for the spectral domain (SD) Green's functions and the spatial domain (SPD) convergence of the Fourier series in the near field. This Chapter is dedicated to the validation of the GPOF approximation, Section 7.1, and the Spatial Domain (SPD) computation of the dyadic component of the Green's function, Section 7.3 ,

\subsection{GPOF Validation}

As first step we derive from the multilayered cylindrical Green's function the free space case. The model will be used also in Section 7.3. Next, the capability of approximation of the GPOF will be tested in the free space case and in the more complex case of a multilayered structure.

\subsubsection{Free Space Model}

We consider first an electrical filament of current directed in $z$ direction, placed at the origin of the circular cylindrical system of coordinate, Figure 13i. We assume the density current distribution Fourier transformable in $z$ and we call $J\left(k_{z}\right)$ its transforation. For convenience, we rewrite (60) 


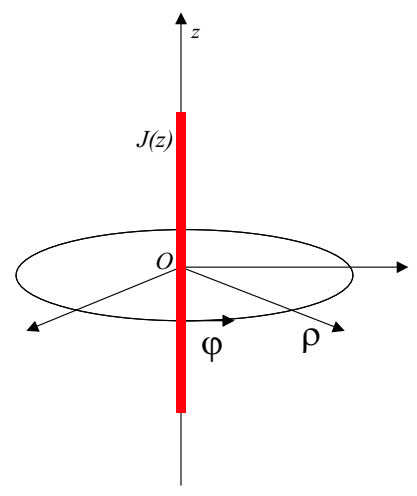

(i) Line source

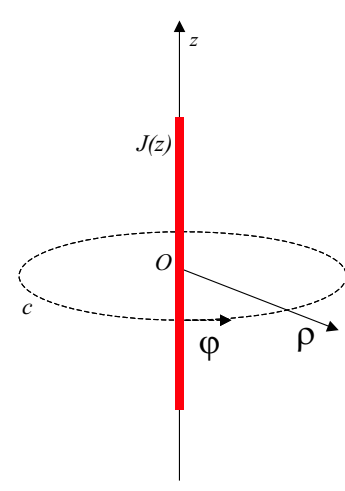

(ii) Ampere integration contour $c$

Figure 13.: In Figure 13i a line source in the center of the cylindrical coordinate system ie represented. In Figure 13ii the Ampere integration contour is shown.

inserting the axial density current dependency in SD

$$
\begin{aligned}
& \bar{\psi}_{\mathcal{F}}=\sum_{n=0}^{\infty} J\left(k_{z}\right)\left[A_{n} H_{n}^{(1)}\left(k_{\rho} \rho\right)+B_{n} H_{n}^{(2)}\left(k_{\rho} \rho\right)\right] \\
& \bar{\psi}_{\mathcal{A}}=\sum_{n=0}^{\infty} J\left(k_{z}\right)\left[C_{n} H_{n}^{(1)}\left(k_{\rho} \rho\right)+D_{n} H_{n}^{(2)}\left(k_{\rho} \rho\right)\right]
\end{aligned}
$$

In the free space case, no reflected waves exist and therefore $A_{n}=0$ and $C_{n}=0$. Moreover, in free space the cylindrical $T E$ and $T M$ waves are uncoupled. Therefore in the following only the radial magnetic scalar vector potential 0 -form will be used. The results are readily usable also for $\varphi$ oriented sources. Since the source is a line placed at of the coordinate system, then (241b) reduces to

$$
\bar{\psi}_{\mathcal{A}}=D_{0} J\left(k_{z}\right) H_{0}^{(2)}\left(k_{\rho} \rho\right)
$$

We consider now the nature of the source and characterize $J\left(k_{z}\right)$ [19]. Using the Ampere theorem to the integration contour $c$, Figure 13ii, we have

$$
\oint_{c} H_{\varphi} \rho \mathrm{d} \varphi=I\left(k_{z}\right)
$$


where $I\left(k_{z}\right)$ is the Fourier transformed of the axial current distribution. Considering the small argument [50] approximation of the Hankel function $\left(\left|k_{\rho} \rho\right| \rightarrow 0\right)$ the $H_{\varphi}$ can be written as follows

$$
H_{\varphi}=-\frac{\partial \psi_{\mathcal{A}}}{\partial \rho} \approx D_{0} J\left(k_{z}\right) \frac{2 j}{\pi \rho}
$$

Inserting (150) into (151) we obtain

$$
\int_{0}^{2 \pi} D_{0} J\left(k_{z}\right) \frac{2 j}{\pi \rho} \rho \mathrm{d} \varphi=4 j D_{0} J\left(k_{z}\right) \approx I\left(k_{z}\right)
$$

and therefore $J\left(k_{z}\right)=I\left(k_{z}\right) / D_{0} 4 j$. The space domain solution of the $\bar{\psi}_{\mathcal{A}}$ is given by the its Fourier back transformation in the following way

$$
\psi_{\mathcal{A}}=\frac{j}{8 \pi} \int_{k_{z}} \frac{I\left(k_{z}\right)}{D_{0}} H_{0}^{(2)}\left(k_{\rho} \rho\right) e^{k_{z} z} \mathrm{~d} k_{z}
$$

If we consider the line source to be an Hertzian dipole of current moment $\mathrm{Il} / D_{0}=1[7,19]$, the density current distribution is SD is given by $J\left(k_{z}\right) / D_{0}=1$. This case is completely described in SD by the GTL model described in Figure 14 and (152) reduces to

$$
\psi_{\mathcal{A}}=\frac{j}{8 \pi} \int_{k_{z}} H_{0}^{(2)}\left(k_{\rho} \rho\right) e^{k_{z} z} \mathrm{~d} k_{z}
$$

It is now straightforward to obtain a model for a point $P=\left(\rho^{\prime}, \varphi^{\prime}, z^{\prime}\right) \neq O$. Using the Addition Theorem for the Hankel functions [27,19], $\bar{\psi}_{\mathcal{A}}$ at the point $P$ in $\mathrm{SD}$ can be written as follows

$$
\bar{\psi}_{\mathcal{A}}= \begin{cases}\sum_{n=-\infty}^{+\infty} H_{n}^{(2)}\left(k_{\rho} \rho^{\prime}\right) J_{n}\left(k_{\rho} \rho\right) e^{j n\left(\varphi-\varphi^{\prime}\right)} & \text { for } \rho<\rho^{\prime} \\ \sum_{n=-\infty}^{+\infty} J_{n}\left(k_{\rho} \rho^{\prime}\right) H_{n}^{(2)}\left(k_{\rho} \rho\right) e^{j n\left(\varphi-\varphi^{\prime}\right)} & \text { for } \rho>\rho^{\prime}\end{cases}
$$

Due to the symmetry of the problem we consider the following proprieties of the Hankel and Bessel functions [50]

$$
\begin{aligned}
J_{-n}(z) & =(-1)^{n} J_{n}(z) \\
H_{-n}^{(2)}(z) & =(-1)^{n} H_{n}^{(2)}(z)
\end{aligned}
$$




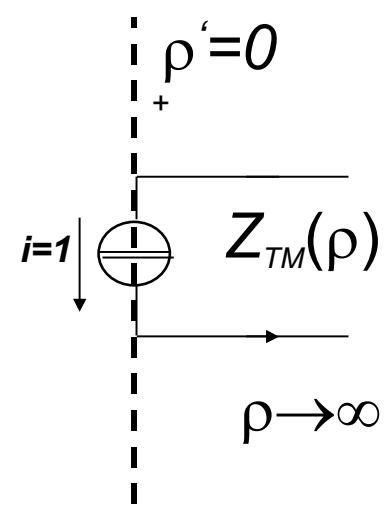

Figure 14.: Equivalent GTL representation for the region $0 \leq \rho<+\infty$.

Considering the case $\rho>\rho^{\prime}$ we can rewrite (154) in the following simplified form

$$
\bar{\psi}_{\mathcal{A}}=J_{0}\left(k_{\rho} \rho^{\prime}\right) H_{0}^{(2)}\left(k_{\rho} \rho\right)+2 \sum_{n=1}^{+\infty} J_{n}\left(k_{\rho} \rho^{\prime}\right) H_{n}^{(2)}\left(k_{\rho} \rho\right) e^{j n\left(\varphi-\varphi^{\prime}\right)}
$$

Since for $z$-oriented Hertzian dipole the main contribute is the $E_{z}$ component of the electric field form, we use it for our model. On the other hands, the $E_{z}$ component of the field is related to the scalar component of the dyadic Green's function $G_{z z}\left(\mathbf{r}, \mathbf{r}^{\prime}\right)$ by (56). Using (154) and (56) we obtain $G_{z z^{\prime}}\left(\mathbf{r}, \mathbf{r}^{\prime}\right)=\left.E_{z}\right|_{\mathcal{J}\left(\mathbf{r}^{\prime}\right)=\mathbf{u}_{\mathbf{z}} \delta\left(\mathbf{r}-\mathbf{r}^{\prime}\right)}=\frac{1}{j \omega \varepsilon}\left(\frac{\partial^{2}}{\partial z^{2}}+k^{2}\right) \psi_{\mathcal{A}}=$ $\frac{1}{4 \pi \omega \varepsilon} \sum_{n=0}^{+\infty} e^{j n\left(\varphi-\varphi^{\prime}\right)} \int_{k_{z}} k_{\rho}^{2} J_{n}\left(k_{\rho} \rho^{\prime}\right) H_{n}^{(2)}\left(k_{\rho} \rho\right) e^{j k_{z}\left(z-z^{\prime}\right)} \mathrm{d} k_{z}$

Defining the

$$
G_{z z^{\prime}}\left(\rho, \rho^{\prime}, k_{z}\right)=G_{n}\left(k_{z}\right)=k_{\rho}^{2} J_{n}\left(k_{\rho} \rho^{\prime}\right) H_{n}^{(2)}\left(k_{\rho} \rho\right)
$$

Inserting (159) into (158) we obtain

$$
G_{z z^{\prime}}\left(\mathbf{r}, \mathbf{r}^{\prime}\right)=\frac{1}{4 \pi \omega \varepsilon} \sum_{n=0}^{+\infty} e^{j n\left(\varphi-\varphi^{\prime}\right)} \int_{k_{z}} G_{n}\left(k_{z}\right) e^{j k_{z}\left(z-z^{\prime}\right)} \mathrm{d} k_{z}
$$


which is a well known cylindrical wave expansion of the Green's function [27].

\subsection{Numerical Results for the GPOF}

In the following section, we test the capability of approximation of the integrand function (102) of the GPOF algorithm.

For evaluating the correctness of the approximation we use as a reference parameter the mean square error (MSE) defined in the following way

$$
M S E=\frac{1}{(N-1)} \sum_{k=0}^{N-1}|G[k]-\hat{G}[k]|^{2}
$$

In Section 7.2.1 the free space case is considered and the analysis is performed for a fixed number of samples $N$. As next test we consider two more complex cases. In Section 7.2 .2 a Hertzian dipole near to a perfect conducting cylindrical reflector is considered. In Section 7.2.3 a Hertzian dipole is confined into a multilayered cylindrical structure. In these cases the algorithm is also tested using as reference parameter the MSE and the analysis is performed as function of the number of samples $\mathrm{N}$.

\subsubsection{Numerical Results for the Free Space case}

As first test, we use as reference function the integrand function of (159), sampled along the complex path of Figure 11 at source point $\rho^{\prime}=0.1 \mathrm{~m}$ and observation $\rho=0.12 \mathrm{~m}$. The approximated function by GPOF is then compared for different mode indexes with the reference function at $\lambda=0.2,0.15,0,1 \mathrm{~m}$. In all cases, the number of total number of samples $N=150$. The results are shown in Figure 27i, 27iii, 27v, We can observe that the MSE behavior does not overcome the threshold of $-20 \mathrm{~dB}$.

\subsubsection{Numerical Results for Perfect Electric Conducting Cylindrical Boundary}

We consider a $z$-oriented Hertzian dipole placed near a Perfect Electric cylinder, (PEC) Figure 16. The PEC is considered to be infinity in $z$ di- 


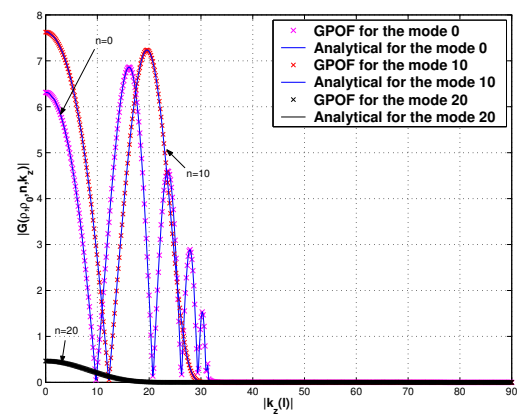

(i) Comparison between the $\left|\bar{G}_{z z}\left(\rho, \rho^{\prime}, k_{z}, n\right)\right|$ and is GPOF approximation at $\lambda=0.20$.

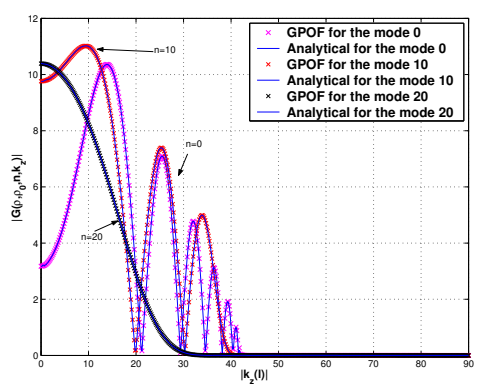

(iii) Comparison between the $\left|\bar{G}_{z z}\left(\rho, \rho^{\prime}, k_{z}, n\right)\right|$ and is GPOF approximation at $\lambda=0.150 \mathrm{~m}$.

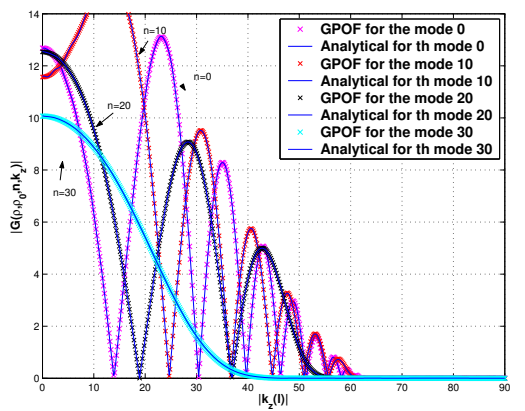

(v) Comparison between the $\left|\bar{G}_{z z}\left(\rho, \rho^{\prime}, k_{z}, n\right)\right|$ and is GPOF approximation at $\lambda=0.1 \mathrm{~m}$.

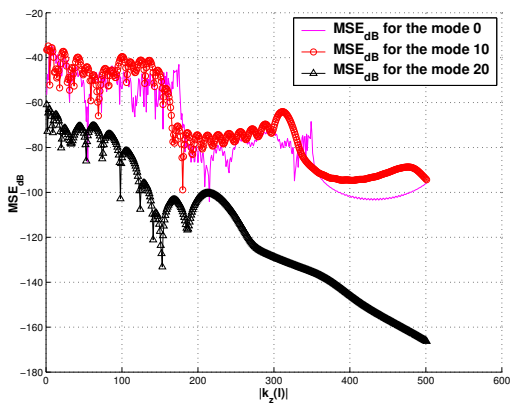

(ii) $M S E_{d B}$ at $\lambda=0.2 \mathrm{~m}$.

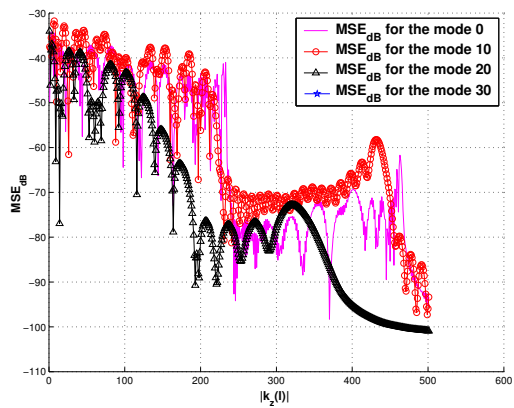

(iv) $M S E_{d B}$ at $\lambda=0.15 \mathrm{~m}$.

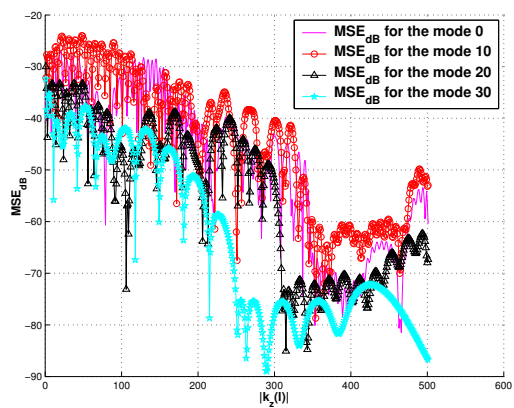

(vi) $M S E_{d B}$ at $\lambda=0.1 \mathrm{~m}$.

Figure 15.: Comparison between the analytical exact solution SD Green s function with the GPOF pole expansion approximation $\left|\bar{G}_{z z}\left(\rho, \rho^{\prime}, k_{z}, n\right)\right|$. 


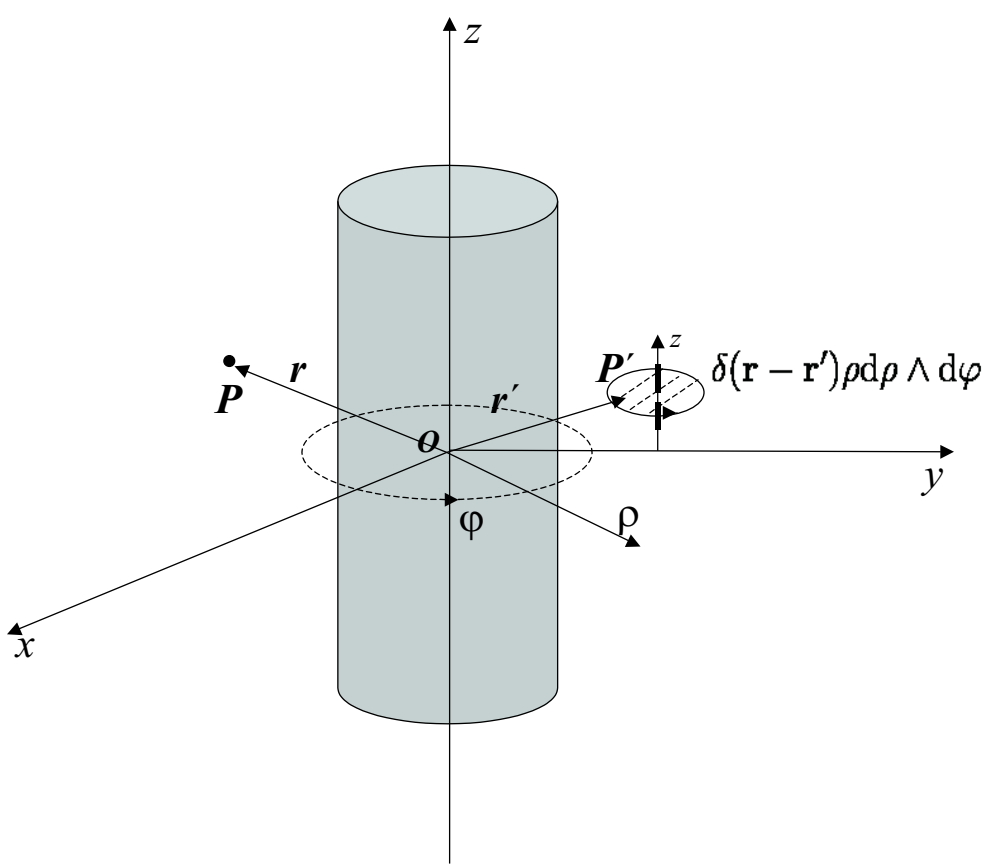

Figure 16.: Hertzian dipole placed near the PEC. 


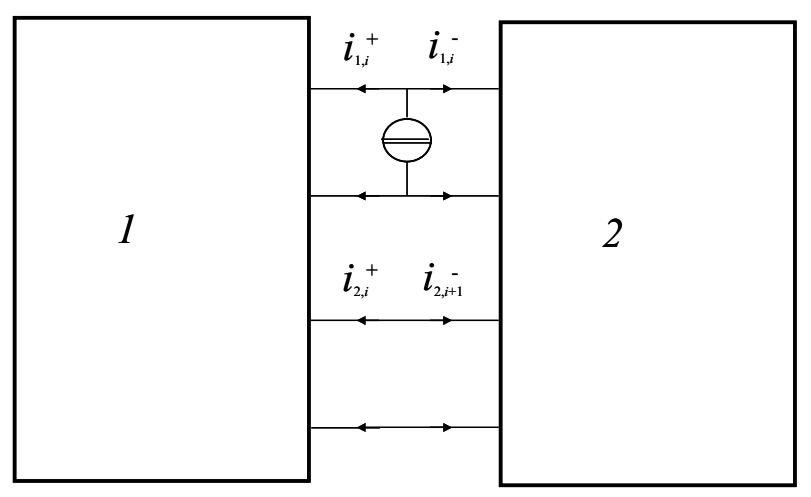

Figure 17.: Equivalent GTL model in SD of the Hertzian dipole and cylindrical reflector.

rection and its radius $\rho_{c}=0.0615 \mathrm{~m}$. The Hertzian dipole is placed at point $P^{\prime}=(0.1015,0,1) \mathrm{m}$ and the observation point $P=(0.1016,0,1)$ $\mathrm{m}$. The Hertzian dipole generates a cylindrical Green's function that can be computed in spectral domain (SD) in the analytical closed form using its equivalent transmission GTL model introduced in this work, Figure 17. The dyadic Green's functions components are computed as in (101) and the component $\bar{G}_{z z}\left(\rho, \rho^{\prime}, k_{z}, n\right)$ is considered. The obtained Green's function is then sampled in the complex $k_{z}$-plane, Figure 11, and then compared with its GPOF approximation in each region of the integration path $\Gamma_{i}$, with $i=1,2,3$, as indicated in Figure 11. For comparing we choose the same number of samples $N$ for each region of the path $\Gamma_{i}$ and two different wavelengths. We may note that changing the number of samples $N=80$ and $N=180$ a very good agreement can be observe in all the 3 region $\Gamma_{i}$ and also in this case the $M S E_{d B}$ does not overcome the threshold of $-15 d B$ in all the cases. The results are summarized in Figures 18, 19, 20, 21. 


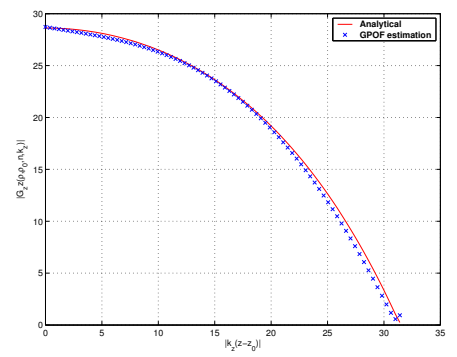

(i) Comparison between the $\left|\bar{G}_{z z}\left(\rho, \rho^{\prime}, k_{z}, 0\right)\right|$ and its GPOF approximation at $\lambda=0.20 \mathrm{~m}$ in $\Gamma_{1}$.

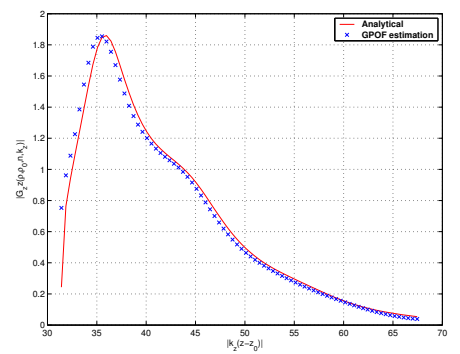

(iii) Comparison between the $\left|\bar{G}_{z z}\left(\rho, \rho^{\prime}, k_{z}, 0\right)\right|$ and its GPOF approximation at $\lambda=0.2 \mathrm{~m}$ in $\Gamma_{2}$.

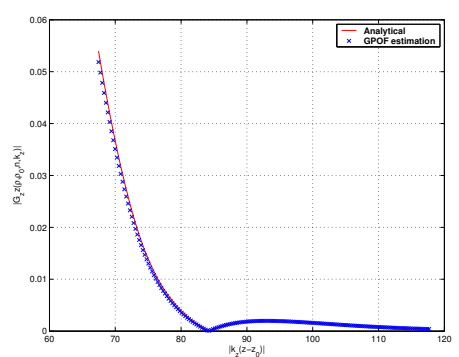

(v) Comparison between the $\left|\bar{G}_{z z}\left(\rho, \rho^{\prime}, k_{z}, 0\right)\right|$ and its GPOF approximation at $\lambda=0.2 \mathrm{~m}$ in $\Gamma_{3}$.

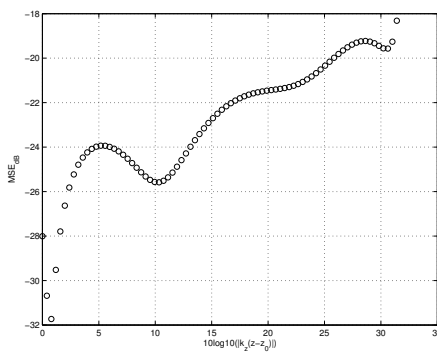

(ii) $M S E_{d B}$ at $\lambda=0.2 \mathrm{~m}$ in $\Gamma_{1}$.

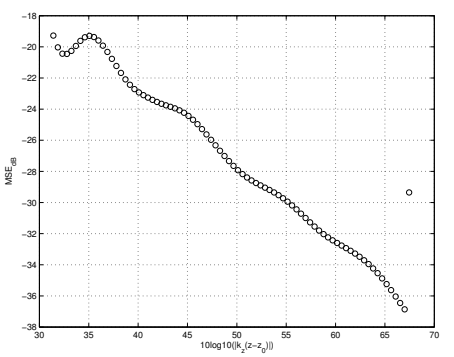

(iv) $M S E_{d B}$ at $\lambda=0.2 \mathrm{~m}$ in $\Gamma_{2}$.

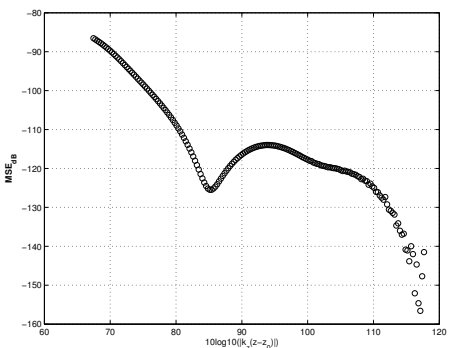

(vi) $M S E_{d B}$ at $\lambda=0.2 \mathrm{~m}$. in $\Gamma_{3}$

Figure 18.: The analytical exact solution SD Green's function $\left|\bar{G}_{z z}\left(\rho, \rho^{\prime}, k_{z}, n\right)\right|$ is sampled into the complex plane indicated in Figure 6.3.2 and is compared its GPOF pole expansion approximation. The number of samples for each $\Gamma_{i}$ $i=1,2,3$ is $N=80, n=0, \rho^{\prime}=0.1015 \mathrm{~m}, \rho=0.11 \mathrm{~m}$ and $\lambda=0.2 \mathrm{~m}$ 


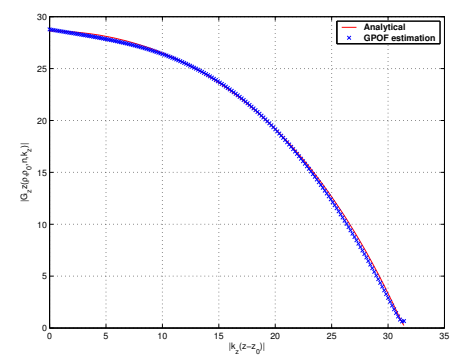

(i) Comparison between the $\left|\bar{G}_{z z}\left(\rho, \rho^{\prime}, k_{z}, 0\right)\right|$ and its GPOF approximation at $\lambda=0.20 \mathrm{~m}$ in $\Gamma_{1}$.

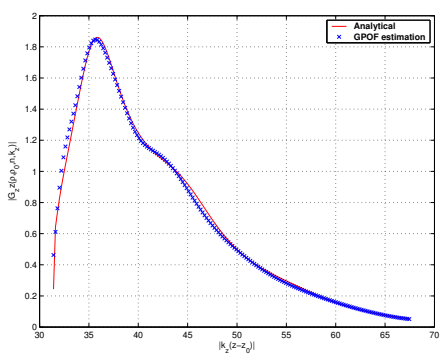

(iii) Comparison between the $\left|\bar{G}_{z z}\left(\rho, \rho^{\prime}, k_{z}, 0\right)\right|$ and its GPOF approximation at $\lambda=0.20 \mathrm{~m}$ in $\Gamma_{2}$.

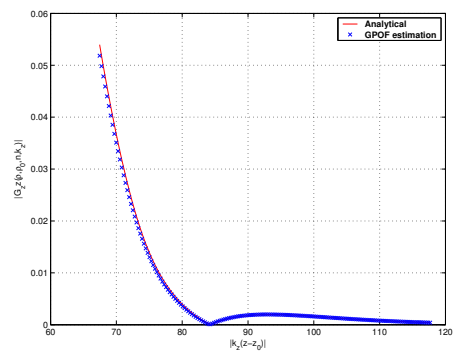

(v) Comparison between the $\left|\bar{G}_{z z}\left(\rho, \rho^{\prime}, k_{z}, 0\right)\right|$ and its GPOF approximation at $\lambda=0.20 \mathrm{~m}$ in $\Gamma_{3}$.

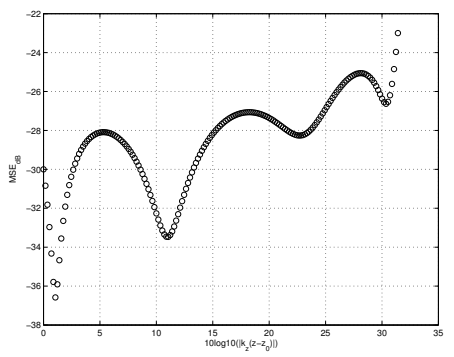

(ii) $M S E_{d B}$ at $\lambda=0.2 \mathrm{~m}$ in $\Gamma_{1}$.

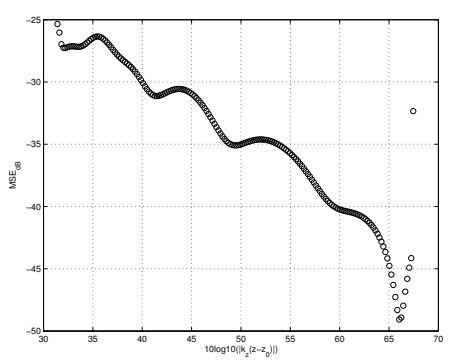

(iv) $M S E_{d B}$ at $\lambda=0.2 \mathrm{~m}$ in $\Gamma_{2}$.

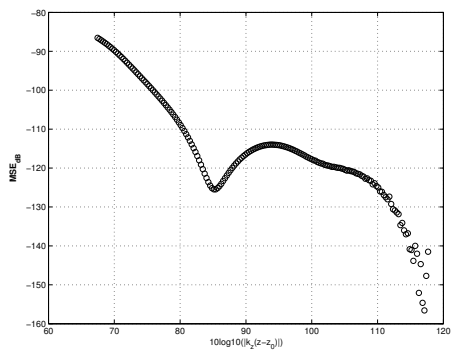

(vi) $M S E_{d B}$ at $\lambda=0.2 \mathrm{~m}$ in $\Gamma_{3}$.

Figure 19.: The analytical exact solution SD Green's function $\left|\bar{G}_{z z}\left(\rho, \rho^{\prime}, k_{z}, n\right)\right|$ is sampled into the complex plane indicated in Figure 6.3 .2 and is compared its GPOF pole expansion approximation. The number of samples for each $\Gamma_{i}$ $i=1,2,3$ is $N=180, n=0, \rho^{\prime}=0.1015 \mathrm{~m}, \rho=0.11 \mathrm{~m}$ and $\lambda=0.2 \mathrm{~m}$. 


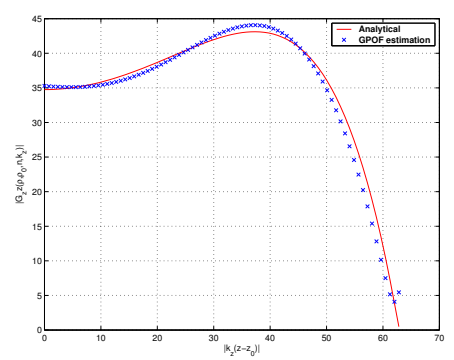

(i) Comparison between the $\left|\bar{G}_{z z}\left(\rho, \rho^{\prime}, k_{z}, 0\right)\right|$ and its GPOF approximation at $\lambda=0.1 \mathrm{~m}$ in $\Gamma_{1}$.

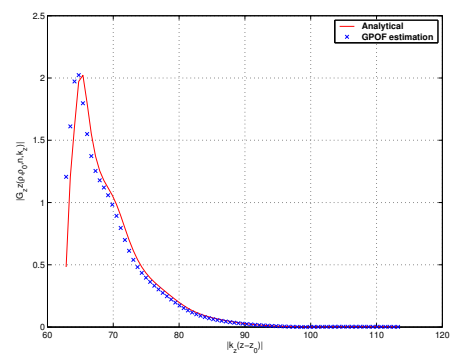

(iii) Comparison between the $\left|\bar{G}_{z z}\left(\rho, \rho^{\prime}, k_{z}, 0\right)\right|$ and its GPOF approximation at $\lambda=0.1 \mathrm{~m}$ in $\Gamma_{2}$.

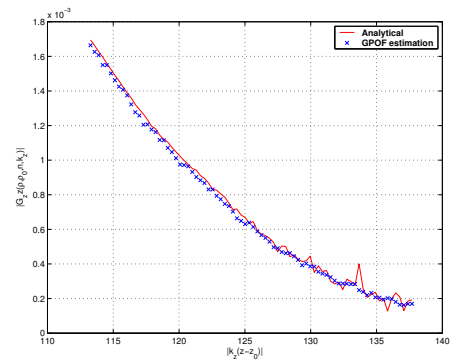

(v) Comparison between the $\left|\bar{G}_{z z}\left(\rho, \rho^{\prime}, k_{z}, 0\right)\right|$ and its GPOF approximation at $\lambda=0.1 \mathrm{~m}$ in $\Gamma_{3}$.

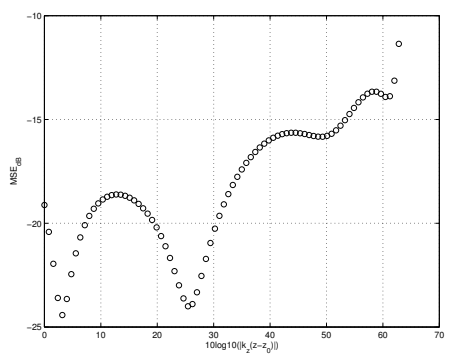

(ii) $M S E_{d B}$ at $\lambda=0.1 \mathrm{~m}$ in $\Gamma_{1}$.

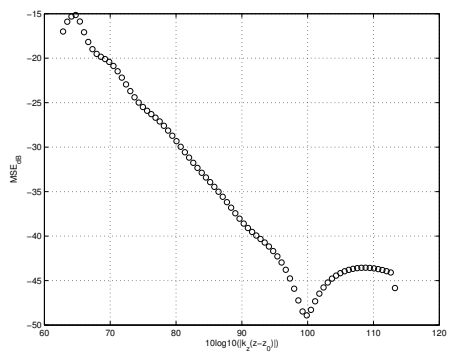

(iv) $M S E_{d B}$ at $\lambda=0.1 \mathrm{~m}$ in $\Gamma_{2}$.

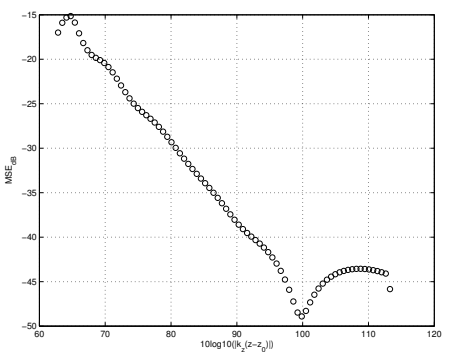

(vi) $M S E_{d B}$ at $\lambda=0.1 \mathrm{~m}$ in $\Gamma_{3}$.

Figure 20.: The analytical exact solution SD Green's function $\left|\bar{G}_{z z}\left(\rho, \rho^{\prime}, k_{z}, n\right)\right|$ is sampled into the complex plane indicated in Figure 6.3.2 and is compared its GPOF pole expansion approximation. The number of samples for each $\Gamma_{i}$ $i=1,2,3$ is $N=80, n=0, \rho^{\prime}=0.1015 \mathrm{~m}, \rho=0.11 \mathrm{~m}$ and $\lambda=0.1 \mathrm{~m}$. 


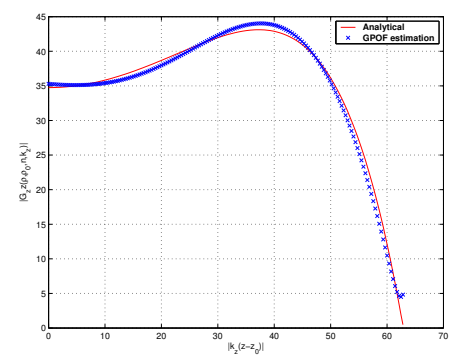

(i) Comparison between the $\left|\bar{G}_{z z}\left(\rho, \rho^{\prime}, k_{z}, 0\right)\right|$ and its GPOF approximation at $\lambda=0.1 \mathrm{~m}$ in $\Gamma_{1}$.

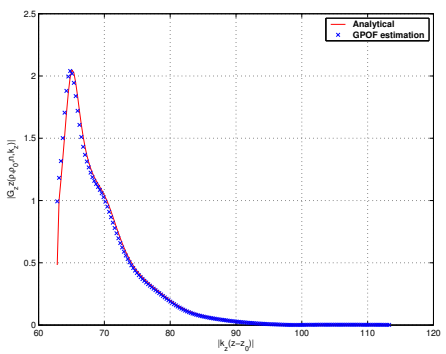

(iii) Comparison between the $\left|\bar{G}_{z z}\left(\rho, \rho^{\prime}, k_{z}, 0\right)\right|$ and its GPOF approximation at $\lambda=0.1 \mathrm{~m}$ in $\Gamma_{2}$.

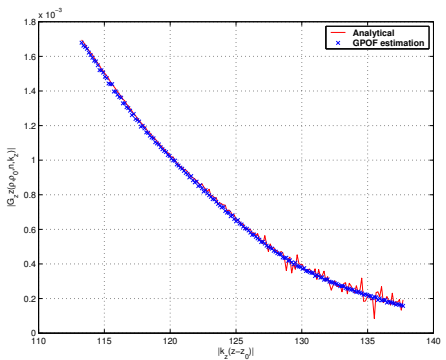

(v) Comparison between the $\left|\bar{G}_{z z}\left(\rho, \rho^{\prime}, k_{z}, 0\right)\right|$ and its GPOF approximation at $\lambda=0.1 \mathrm{~m}$ in $\Gamma_{3}$.

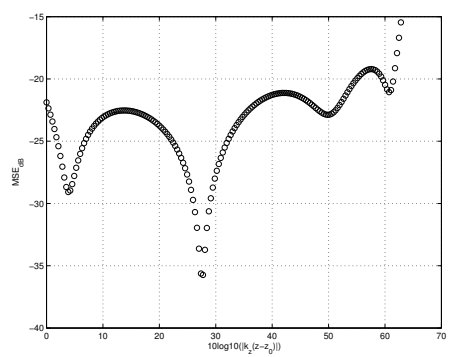

(ii) $M S E_{d B}$ at $\lambda=0.1 \mathrm{~m}$ in $\Gamma_{1}$.

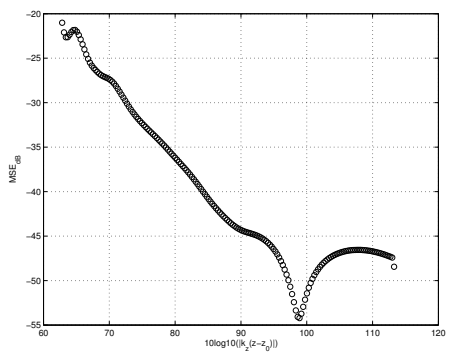

(iv) $M S E_{d B}$ at $\lambda=0.1 \mathrm{~m}$ in $\Gamma_{2}$.

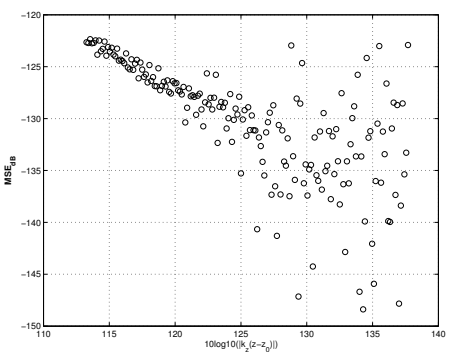

(vi) $M S E_{d B}$ at $\lambda=0.1 \mathrm{~m}$ in $\Gamma_{3}$.

Figure 21.: The analytical exact solution SD Green's function $\left|\bar{G}_{z z}\left(\rho, \rho^{\prime}, k_{z}, n\right)\right|$ is sampled into the complex plane indicated in Figure 6.3.2 and is compared its GPOF pole expansion approximation. The number of samples for each $\Gamma_{i}$ $i=1,2,3$ is $N=180, n=0, \rho^{\prime}=0.1015 \mathrm{~m}, \rho=0.11 \mathrm{~m}$ and $\lambda=0.1 \mathrm{~m}$. 


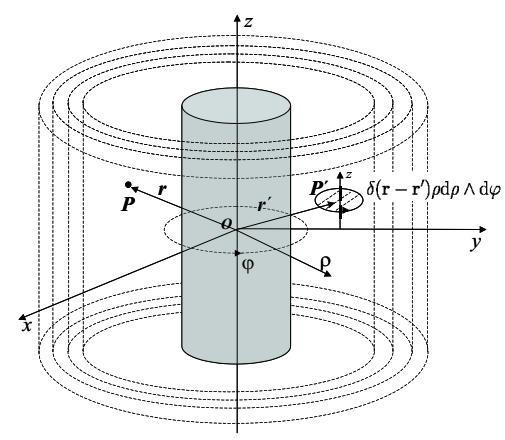

(i) Source position inside the multilayered cylindrical structure.

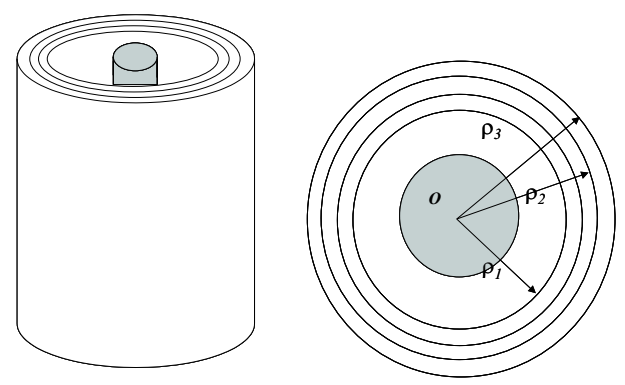

(ii) Structure view.

Figure 22.: In Figure is reported the layout of the structure.

\subsubsection{Numerical Results for Multilayered Cylindrical Structure}

We consider a $z$-oriented Hertzian dipole confined inside a multilayered cylindrical structure Figure 22i, 22ii. The underlined multilayered structure is assumed to be infinitely extended in $z$-direction and composed of 6 layers as depicted in Figure 22ii. The radii of the dielectric layers are $\rho_{c}=0.0615$ $\mathrm{m}, \rho_{1}=0.2450 \mathrm{~m}, \rho_{2}=0.2490 \mathrm{~m}, \rho_{3}=0.2700 \mathrm{~m}, \rho_{4}=0.274, \rho_{5} \rightarrow+\infty$, Figure 22ii. The layer 1 is a PEC the layer 2, 3, 4, 5, are non-magnetic materials described by the following dielctric constants: $\varepsilon_{2}=1, \varepsilon_{3}=4.5$, $\varepsilon_{4}=1.1, \varepsilon_{5}=4.5, \varepsilon_{6}=1$, Figure 22ii. The Hertzian dipole is embedded in the layer 2 at point $P^{\prime}=(0.1015,0,1) \mathrm{m}$ and the observation point $P=(0.1016,0,1) \mathrm{m}$. As in the previous example the cylindrical Green's functions that can be computed in spectral domain (SD) in the analytical closed form using its equivalent transmission GTL model introduced in this work, Figure 23. As in the previous example the component $\bar{G}_{z z}\left(\rho, \rho^{\prime}, k_{z}, n\right)$ is considered and sampled in the complex $k_{z}$-plane. Similarly to the previous case for comparing purposes, we choose the same number of samples $N$ for each region of the path $\Gamma_{i}$ and two different wavelengths. In this case, for $N=80$ the $M S E_{d B}<-10 d B$ while for $N=180$ the $M S E_{d B}$ is below 


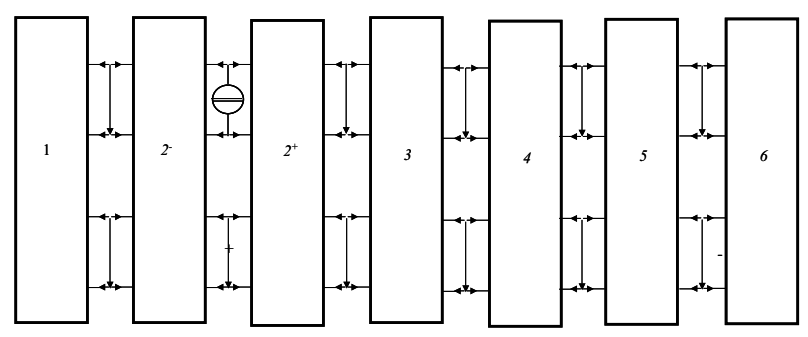

Figure 23.: Equivalent GTL model in SD of the Hertzian dipole and three layered structure.

$-15 d B$. The results are summarized in Figures 24, 25,26, 27.

\subsection{Nearfield Green's Function}

The usage of the Green's function for Method of Moment (MoM) and other electromagnetic applications, requires the knowledge of the nearfield. It is known that infinitesimal concentrated sources create singular fields. It is remarkable to point out that in literature a large production of scientific publications on the topic has been occurring since 1960s [39,40,41]. Since the understating of the singularities is essential for the use of the dyadic Green's function in numerical methods, in the following we will investigate the singularities-type in the source region and develop a simple and intuitive approach to deal with them in our algorithm. In Section 7.3.1 the problem of the singularities will be formulated. In Section 7.3.2 the problem of the singularities for the cylindrical waves expansion is discussed. In Section 7.3.3, we summarize the description of dipole in free space and offset from the origin used as reference. In Section 7.3.4 we compare the results obtain numerically and the analytical models and the limitations are discussed. In Section 7.3.5 we derive an advanced reference model and the improvements are then shown and discussed in Section 7.3.7. 


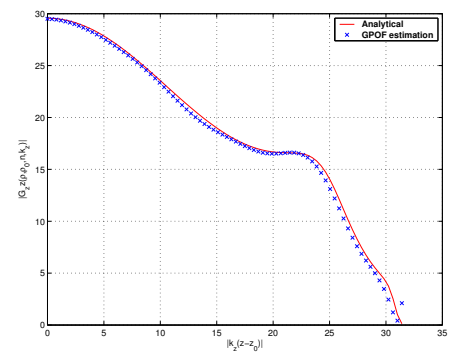

(i) Comparison between the $\left|\bar{G}_{z z}\left(\rho, \rho^{\prime}, k_{z}, 0\right)\right|$ and its GPOF approximation at $\lambda=0.2 \mathrm{~m}$ in $\Gamma_{1}$.

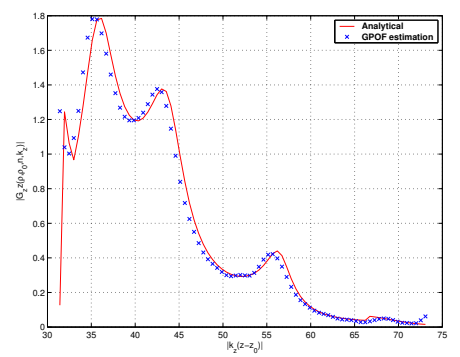

(iii) Comparison between the $\left|\bar{G}_{z z}\left(\rho, \rho^{\prime}, k_{z}, 0\right)\right|$ and its GPOF approximation at $\lambda=0.1 \mathrm{~m}$ in $\Gamma_{2}$.

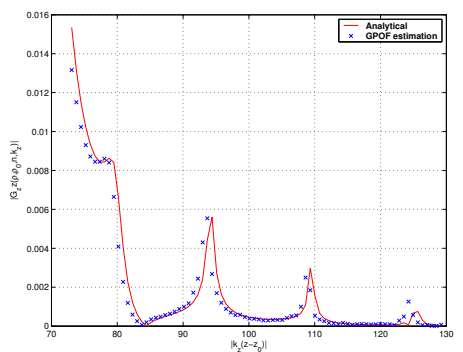

(v) Comparison between the $\left|\bar{G}_{z z}\left(\rho, \rho^{\prime}, k_{z}, 0\right)\right|$ and its GPOF approximation at $\lambda=0.1 \mathrm{~m}$ in $\Gamma_{3}$.

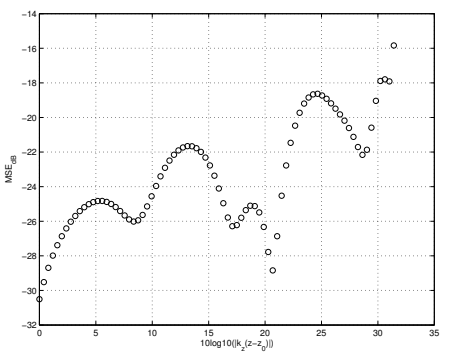

(ii) $M S E_{d B}$ at $\lambda=0.2 \mathrm{~m}$ in $\Gamma_{1}$.

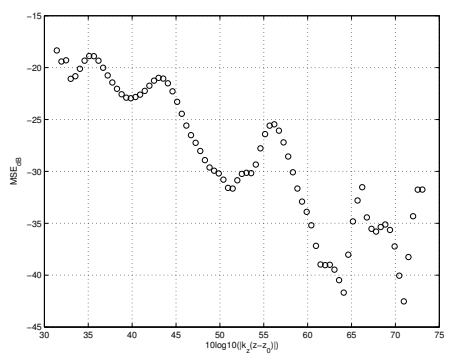

(iv) $M S E_{d B}$ at $\lambda=0.2 \mathrm{~m}$ in $\Gamma_{2}$.

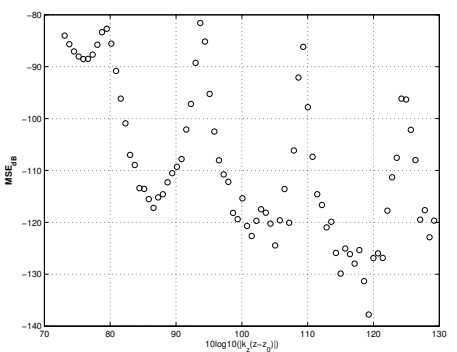

(vi) $M S E_{d B}$ at $\lambda=0.2 \mathrm{~m}$ in $\Gamma_{3}$.

Figure 24.: The analytical exact solution SD Green's function $\left|\bar{G}_{z z}\left(\rho, \rho^{\prime}, k_{z}, n\right)\right|$ is sampled into the complex plane indicated in Figure 6.3.2 and is compared its GPOF pole expansion approximation. The number of samples for each $\Gamma_{i}$ $i=1,2,3$ is $N=80, n=0, \rho^{\prime}=0.1015 \mathrm{~m}, \rho=0.11 \mathrm{~m}$ and $\lambda=0.2 \mathrm{~m}$ with radome. 


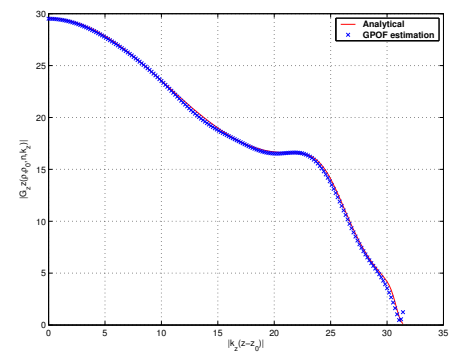

(i) Comparison between the $\left|\bar{G}_{z z}\left(\rho, \rho^{\prime}, k_{z}, 0\right)\right|$ and its GPOF approximation at $\lambda=0.2 \mathrm{~m}$ in $\Gamma_{1}$.

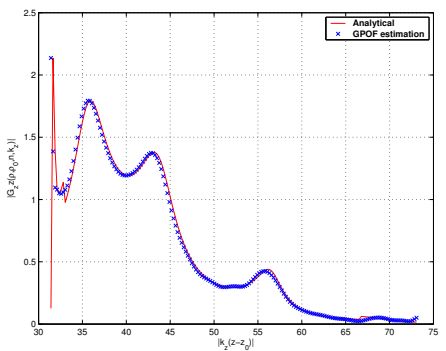

(iii) Comparison between the $\left|\bar{G}_{z z}\left(\rho, \rho^{\prime}, k_{z}, 0\right)\right|$ and its GPOF approximation at $\lambda=0.2 \mathrm{~m}$ in $\Gamma_{2}$.

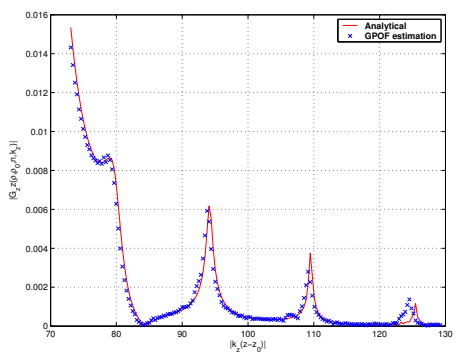

(v) Comparison between the $\left|\bar{G}_{z z}\left(\rho, \rho^{\prime}, k_{z}, n\right)\right|$ and is GPOF approximation at $\lambda=0.1 \mathrm{~m}$.

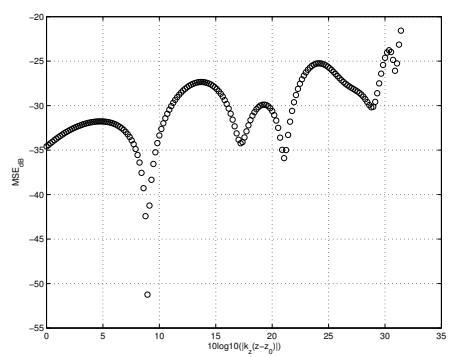

(ii) $M S E_{d B}$ at $\lambda=0.2 \mathrm{~m}$ in $\Gamma_{1}$

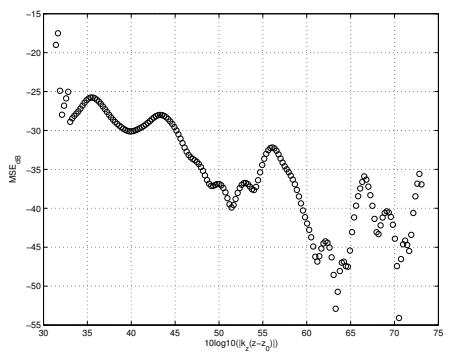

(iv) $M S E_{d B}$ at $\lambda=0.2 \mathrm{~m}$ in $\Gamma_{2}$

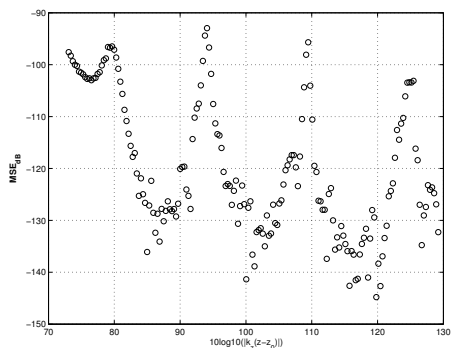

(vi) $M S E_{d B}$ at $\lambda=0.2 \mathrm{~m}$ in $\Gamma_{3}$

Figure 25.: The analytical exact solution SD Green's function $\left|\bar{G}_{z z}\left(\rho, \rho^{\prime}, k_{z}, n\right)\right|$ is sampled into the complex plane indicated in Figure 6.3.2 and is compared its GPOF pole expansion approximation. The number of samples for each $\Gamma_{i}$ $i=1,2,3$ is $N=180, n=0, \rho^{\prime}=0.1015 \mathrm{~m}, \rho=0.11 \mathrm{~m}$ and $\lambda=0.2 \mathrm{~m}$ with radome. 


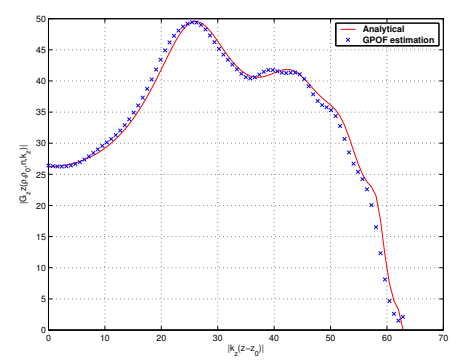

(i) Comparison between the $\left|\bar{G}_{z z}\left(\rho, \rho^{\prime}, k_{z}, 0\right)\right|$ and is GPOF approximation at $\lambda=0.1 \mathrm{~m}$ in $\Gamma_{1}$.

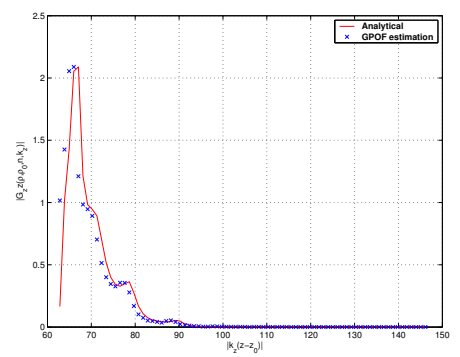

(iii) Comparison between the $\left|\bar{G}_{z z}\left(\rho, \rho^{\prime}, k_{z}, 0\right)\right|$ and is GPOF approximation at $\lambda=0.1 \mathrm{~m}$ in $\Gamma_{2}$.

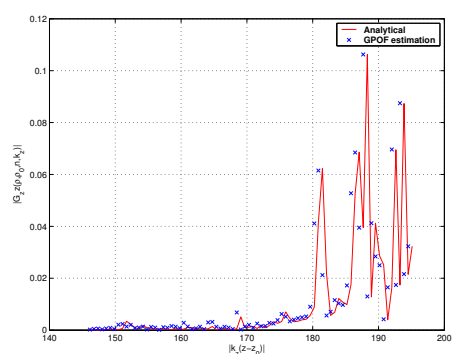

(v) Comparison between the $\left|\bar{G}_{z z}\left(\rho, \rho^{\prime}, k_{z}, 0\right)\right|$ and is GPOF approximation at $\lambda=0.1 \mathrm{~m}$ in $\Gamma_{3}$.

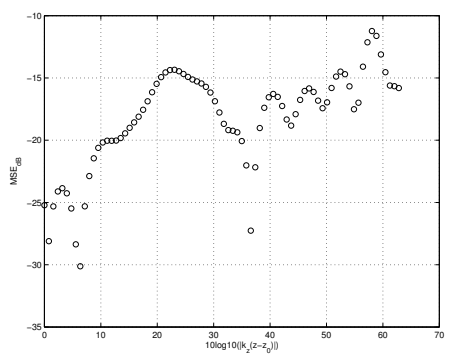

(ii) $M S E_{d B}$ at $\lambda=0.1 \mathrm{~m}$ in $\Gamma_{1}$.

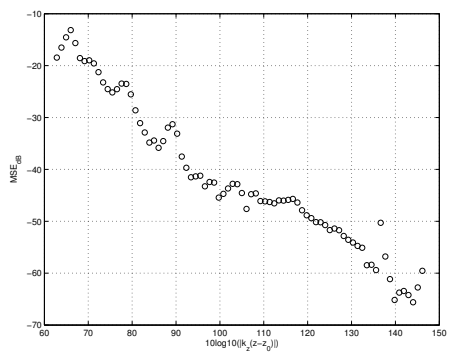

(iv) $M S E_{d B}$ at $\lambda=0.2 \mathrm{~m}$ in $\Gamma_{2}$.

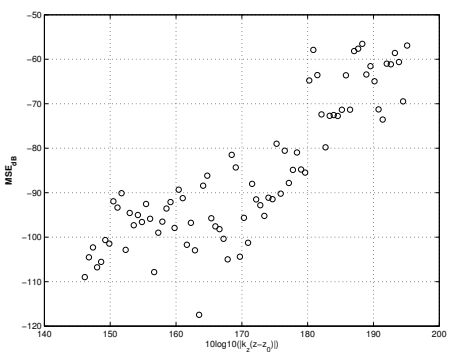

(vi) $M S E_{d B}$ at $\lambda=0.1 \mathrm{~m}$ in $\Gamma_{3}$.

Figure 26.: The analytical exact solution SD Green's function $\left|\bar{G}_{z z}\left(\rho, \rho^{\prime}, k_{z}, n\right)\right|$ is sampled into the complex plane indicated in Figure 6.3.2 and is compared its GPOF pole expansion approximation. The number of samples for each $\Gamma_{i}$ $i=1,2,3$ is $N=80, n=0, \rho^{\prime}=0.1015 \mathrm{~m}, \rho=0.11 \mathrm{~m}$ and $\lambda=0.1 \mathrm{~m}$ with radome. 


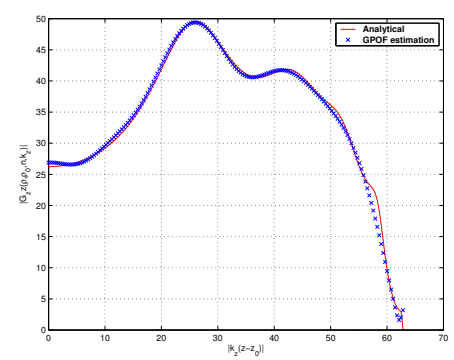

(i) Comparison between the $\left|\bar{G}_{z z}\left(\rho, \rho^{\prime}, k_{z}, 0\right)\right|$ and is GPOF approximation at $\lambda=0.1 \mathrm{~m}$ in $\Gamma_{2}$.

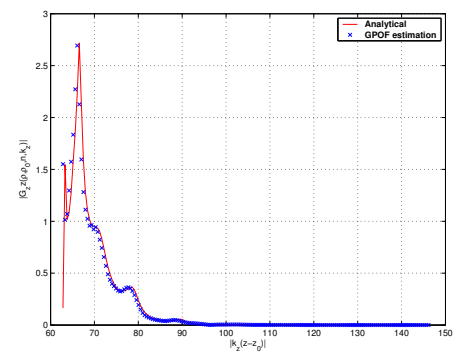

(iii) Comparison between the $\left|\bar{G}_{z z}\left(\rho, \rho^{\prime}, k_{z}, 0\right)\right|$ and is GPOF approximation at $\lambda=0.1 \mathrm{~m}$ in $\Gamma_{2}$.

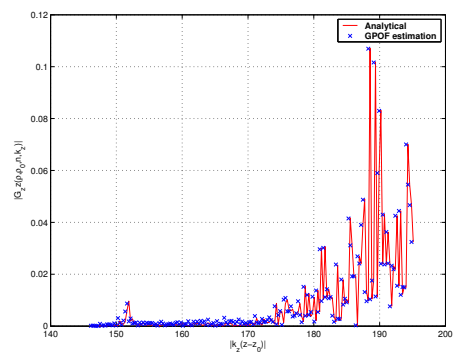

(v) Comparison between the $\left|\bar{G}_{z z}\left(\rho, \rho^{\prime}, k_{z}, 0\right)\right|$ and is GPOF approximation at $\lambda=0.1 \mathrm{~m}$ in $\Gamma_{3}$.

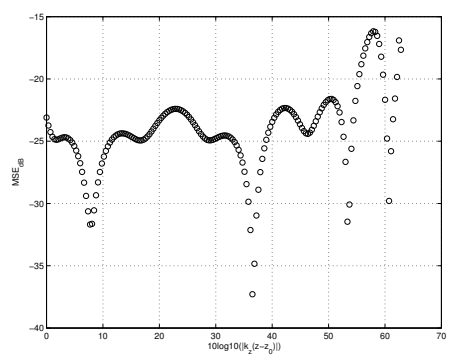

(ii) $M S E_{d B}$ at $\lambda=0.1 \mathrm{~m}$ in $\Gamma_{1}$

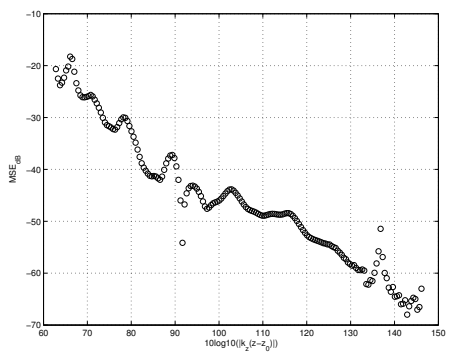

(iv) $M S E_{d B}$ at $\lambda=0.1 \mathrm{~m}$ in $\Gamma_{2}$

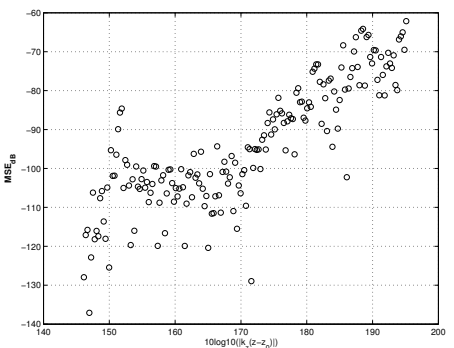

(vi) $M S E_{d B}$ at $\lambda=0.1 \mathrm{~m}$ in $\Gamma_{3}$

Figure 27.: The analytical exact solution SD Green's function $\left|\bar{G}_{z z}\left(\rho, \rho^{\prime}, k_{z}, n\right)\right|$ is sampled into the complex plane indicated in Figure 6.3.2 and is compared its GPOF pole expansion approximation. The number of samples for each $\Gamma_{i}$ $i=1,2,3$ is $N=180, n=0, \rho^{\prime}=0.1015 \mathrm{~m}, \rho=0.11 \mathrm{~m}$ and $\lambda=0.1 \mathrm{~m}$ with radome. 


\subsubsection{Singularities-Type in the Source Region}

We consider the free space case since the singularity-source type does not change with the structure. As was previously described with (199), the field can be written as linear combination of current in the integral form as follows [21]

$$
\mathcal{E}(\mathbf{r})=j \omega \mu \int_{V^{\prime}} \mathcal{G}_{e}\left(\mathbf{r}, \mathbf{r}^{\prime}\right) \wedge \mathcal{J}\left(\mathbf{r}^{\prime}\right)
$$

which is defined for $\mathbf{r} \neq \mathbf{r}^{\prime}$. A more complete representation of the integral equation can be given using the theory of distribution [41] in which also the distance $\left|\mathbf{r}-\mathbf{r}^{\prime}\right|<\varepsilon$ with $\varepsilon \rightarrow 0$ can be considered. In particular for the dyadic Green's function $\mathcal{G}_{e}\left(\mathbf{r}, \mathbf{r}^{\prime}\right)$ we introduce the following distribution

$$
\mathcal{G}_{e}\left(\mathbf{r}, \mathbf{r}^{\prime}\right)=\mathbf{P V}_{V^{\prime}} \mathcal{G}_{e}\left(\mathbf{r}, \mathbf{r}^{\prime}\right)+\mathcal{G}_{e}\left(\mathbf{r}, \mathbf{r}^{\prime}\right)^{i}
$$

where $\mathcal{G}_{e}\left(\mathbf{r}, \mathbf{r}^{\prime}\right)^{i}$ is the contribution form the current in the small volume $V^{\prime}$ containing the source and $\mathbf{P V}_{V}^{\prime} \mathcal{G}_{e}\left(\mathbf{r}, \mathbf{r}^{\prime}\right)$ is the principal value of the integral (162) in the volume $V^{\prime}$. Accordingly with the theory of distribution, the contribution at distance $\left|\mathbf{r}-\mathbf{r}^{\prime}\right|<\epsilon$ can be assigned to an interior dyadic defined as follows [41]

$$
\mathcal{G}_{e}\left(\mathbf{r}, \mathbf{r}^{\prime}\right)^{i}=\frac{\mathcal{J}(\mathbf{r}) \mathbf{L}_{V^{\prime}}}{k^{2}}
$$

where $\mathbf{L}_{V^{\prime}}$ a dyadic source [39,41] that depends only on the geometry of the source. The physical interpretation was given in [39] and states that the value of $\mathbf{L}_{V^{\prime}}$ is the electric field measured removing an infinitesimal volume of a given shape. Inserting (164) and (163) into (162) we obtain a definition for the integral equation including also the distance $\left|\mathbf{r}-\mathbf{r}^{\prime}\right|=0$

$$
\mathcal{E}(\mathbf{r})=j \omega \mu \mathbf{P} \mathbf{V}_{V^{\prime}} \int_{V^{\prime}} \mathcal{G}_{e}\left(\mathbf{r}, \mathbf{r}^{\prime}\right) \wedge \mathcal{J}\left(\mathbf{r}^{\prime}\right)+\frac{\mathcal{J}(\mathbf{r}) \mathbf{L}_{V^{\prime}}}{j \omega \mu \varepsilon}
$$

the value of $\mathbf{L}_{V^{\prime}}$, which depends only on the shape of the volume, is known analytically in some special cases i.e. cube, spherical and cylindrical volumes. The procedure to compute it analytically has been extensively treated in literature $[39,40,41]$ and in the following the result for the cylindrical case is only reported for completeness, see Figure 28

$$
\begin{gathered}
\mathbf{L}_{V^{\prime}}=\frac{1}{2} \cos \theta_{0} \mathbf{I}_{x y}+\left(1-\cos \theta_{0}\right) \mathrm{d} z \mathrm{~d} z^{\prime} \\
\mathbf{I}_{x y}=\mathrm{d} x \mathrm{~d} x^{\prime}+\mathrm{d} y \mathrm{~d} y^{\prime}
\end{gathered}
$$




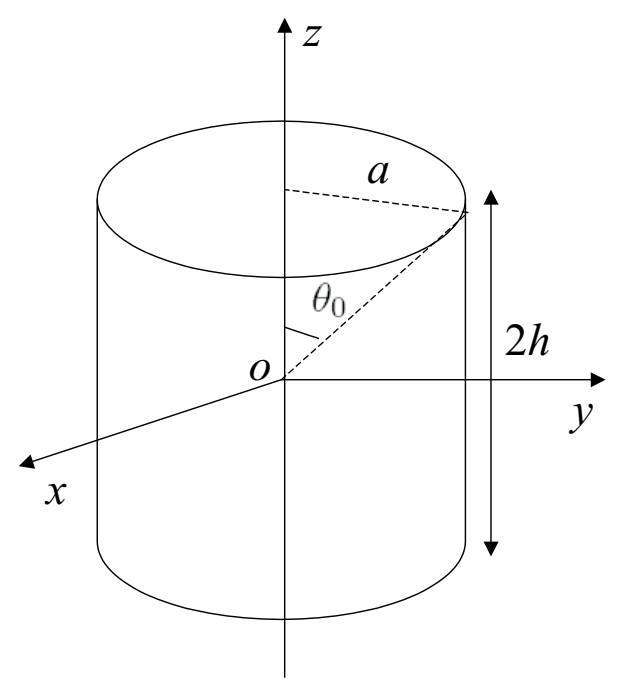

Figure 28.: Circular cylinder dyad L.

Conversely, from the numerical view point a different approach is needed. In fact the result (166) is rigorous but difficult to be used as reference model. That will be extensively discussed in the next two Sections. In Section 7.3.5 an alternative reference model for numerical near field computation will be introduced.

\subsubsection{Singularities in the Cylindrical Waves Expansion}

If the Green's function is expanded into cylindrical waves, the singularity problem, it is understood in terms of series convergence. To investigate the problem we consider the dyadic component of the Green's function $G_{z z^{\prime}}^{i j}\left(\mathbf{r}, \mathbf{r}^{\prime}\right)$. Assuming that it is allowable to exchange the series with the 
integral we obtain

$$
\begin{gathered}
G_{z z^{\prime}}^{i j}\left(\mathbf{r}, \mathbf{r}^{\prime}\right)=\frac{j}{8 \pi} \int_{k_{z}} S_{n}\left(\rho, \rho^{\prime}, \varphi, \varphi^{\prime}, k_{\rho_{i}}\right) e^{-j k_{z}\left(z-z^{\prime}\right)} \mathrm{d} k_{z} \\
S_{n}\left(\rho, \rho^{\prime}, \varphi, \varphi^{\prime}, k_{\rho_{i}}\right)= \\
\sum_{n=0}^{\infty} \frac{k_{\rho_{i}}^{2}}{j \omega \varepsilon_{i}}\left(A_{n}^{i}\left(\rho^{\prime}\right) H_{n}^{(1)}\left(k_{\rho_{i}} \rho_{i}\right)+B_{n}^{i}\left(\rho^{\prime}\right) H_{n}^{(2)}\left(k_{\rho_{i}} \rho_{i}\right)\right) e^{-j n\left(\varphi-\varphi^{\prime}\right)}
\end{gathered}
$$

where $k_{\rho_{i}}=\sqrt{k_{i}^{2}-k_{z}^{2}}$. For simplicity of notation we rename

$$
S_{n}=S_{n}\left(\rho, \rho^{\prime}, \varphi, \varphi^{\prime}, k_{\rho_{i}}\right)
$$

We assume that $\varphi-\varphi^{\prime} \neq 0$ and $z-z^{\prime} \neq 0$ and $\left|\rho-\rho^{\prime}\right|<\varepsilon$. For $\left|k_{\rho_{i}} \rho_{i}\right| \rightarrow 0$ the series $S_{n}$ forms a divergent series due to the singular behavior of the Hankel functions. For large value of the Hankel function argument $\left|k_{\rho_{i}} \rho_{i}\right| \rightarrow+\infty$ the Hankel functions tend to behave asymptotically like dumped exponential functions. The complex modal amplitudes $A_{n}\left(\rho^{\prime}\right)$ and $B_{n}\left(\rho^{\prime}\right)$, computed by matching the boundary conditions, represent the impressed excitation to each mode at a given frequency. That means that their contribute tend to vanish while $n \rightarrow+\infty$. In this case $S_{n}$ forms a fast convergent series. Therefore in order to have a fast convergent series we need to keep the argument of the Hankel functions large enough introducing lossy accordingly to what has been discussed in the Chapter 6. If $\left|\varphi-\varphi^{\prime}\right|<\varepsilon$ and $z-z^{\prime} \neq 0$ then $S_{n}$ reduces to

$$
S_{n}\left(\rho, \rho^{\prime}, \varphi, \varphi^{\prime}, k_{\rho_{i}}\right)=\sum_{n=0}^{\infty} \frac{k_{\rho_{i}}^{2}}{j \omega \varepsilon_{i}}\left(A_{n}^{i}\left(\rho^{\prime}\right) H_{n}^{(1)}\left(k_{\rho_{i}} \rho_{i}\right)+B_{n}^{i}\left(\rho^{\prime}\right) H_{n}^{(2)}\left(k_{\rho_{i}} \rho_{i}\right)\right)
$$

Also in this case (169) forms a convergent series and the same considerations previously discussed can be made.

\subsubsection{Source Singularity-Type Analysis for an Infinitesimal Electric Dipole Model.}

Our purpose in this Section is to derive a reference model for the SPD computation of the dyadic Green's function. Since severe numerical problems can appear for near field computation we shall concentrate our attention on it. In Section 7.1, staring from the multilayered cylindrical Green's functions (102), we have derived the known free space case. In this Section, using 
the results of Section 7.1 we derive an analytical closed form for the free space case usable as reference model. Making use of the theorem of uniqueness of the solution one can write the following identities i.e. Sommerfeld Identities (SI) [27,42, 19]

$$
\begin{gathered}
\psi_{\mathcal{A}}=\frac{j}{8 \pi} \int_{k_{z}} H_{0}^{(2)}\left(k_{\rho} \rho\right) e^{j k_{z} z} \mathrm{~d} k_{z} \\
\frac{e^{-j k r}}{4 \pi r}=\frac{j}{8 \pi} \int_{k_{z}} H_{0}^{(2)}\left(k_{\rho} \rho\right) e^{j k_{z} z} \mathrm{~d} k_{z}
\end{gathered}
$$

Following the procedure of the Section 7.1 of can also easily verify that for $\mathbf{r}^{\prime} \neq O$ and for $\rho>\rho^{\prime}$ the following identities are verified

$$
\frac{e^{-j k\left|\mathbf{r}-\mathbf{r}^{\prime}\right|}}{4 \pi\left|\mathbf{r}-\mathbf{r}^{\prime}\right|}=\frac{j}{8 \pi} \sum_{n=1}^{+\infty} \int_{k_{z}} J_{n}\left(k_{\rho} \rho^{\prime}\right) H_{n}^{(2)}\left(k_{\rho} \rho\right) e^{j n\left(\varphi-\varphi^{\prime}\right)} e^{j k_{z}\left(z-z^{\prime}\right)} \mathrm{d} k_{z}
$$

Using the identities (171) and (172) we can compute for the free space case an analytical closed form for the small electric dipole usable as reference. We assume now the dipole place at $P^{\prime}=O$ and the observation point is $P=(\rho, \varphi, z)$. In this case the magnetic vector potential form $\mathcal{A}$ generated by the $z$-oriented small electric dipole can be written as follows $[28,21]$

$$
\underline{\mathcal{A}}=\psi_{\mathcal{A}}(\mathbf{r}, 0) \mathrm{d} z=\frac{\mu I_{0} l}{4 \pi \mathbf{r}} e^{-j k \mathbf{r}} \mathrm{d} z
$$

where $\mathbf{r}$ is the distance of the observation point from the location of the dipole and $\psi_{\mathcal{A}}$ is 0 double form Green's function. Inserting (173) into (48) we obtain

$$
\begin{aligned}
& H_{r}=0 \\
& H_{\varphi}=j \frac{k I_{0} l \sin \theta}{4 \pi r}\left[1+\frac{1}{j k r}\right] e^{-j k r} \\
& H_{\theta}=0 \\
& E_{r}=\eta \frac{I_{0} l \cos \theta}{2 \pi r^{2}}\left[1+\frac{1}{j k r}\right] e^{-j k r} \\
& E_{\varphi}=0 \\
& E_{\theta}=j \eta \frac{k I_{0} l \sin \theta}{4 \pi r}\left[1+\frac{1}{j k r}-\frac{1}{(k r)^{2}}\right] e^{-j k r}
\end{aligned}
$$


Transforming (174a) to (174f), into cylindrical coordinates $(\rho, \varphi, z)$, we obtain the $E_{z}$ component

$$
E_{z}=\frac{1}{j \omega \varepsilon} I_{0} l \frac{e^{-j k R}}{2 \pi R^{5}}\left[(1+j k R)\left(2 R^{2}-3 \rho^{2}\right)+(k \rho R)^{2}\right]
$$

where

$$
R=\sqrt{\rho^{2}+z^{2}}
$$

We assume now the dipole place at $P^{\prime}=\left(\rho^{\prime}, \varphi^{\prime}, 0\right)$ and the observation point is $P=(\rho, \varphi, z)$. In this case the magnetic vector potential form $\mathcal{A}$ generated by the $z$-oriented small electric dipole can be written as follows

$$
\underline{\mathcal{A}}=\psi_{\mathcal{A}} \mathrm{d} z=\frac{e^{-j k\left|\mathbf{r}-\mathbf{r}^{\prime}\right|}}{4 \pi\left|\mathbf{r}-\mathbf{r}^{\prime}\right|} \mathrm{d} z
$$

Similar as for the dipole in the origin, we get [28]

$$
E_{z}=\frac{1}{j \omega \varepsilon} I_{0} l \frac{e^{-j k R^{\prime}}}{2 \pi R^{\prime 5}}\left[\left(1+j k R^{\prime}\right)\left(2 R^{\prime 2}-3 \rho^{\prime 2}\right)+\left(k \rho^{\prime} R^{\prime}\right)^{2}\right]
$$

with

$$
\begin{aligned}
\rho^{\prime} & =\sqrt{\left(\rho \cos \left(\varphi-\varphi^{\prime}\right)-\rho^{\prime}\right)^{2}+\left(\rho \sin \left(\varphi-\varphi^{\prime}\right)\right)^{2}} \\
R^{\prime} & =\sqrt{\rho^{\prime 2}+z^{2}}
\end{aligned}
$$

The latter represents the classical closed form expression of $E_{z}$ component of the the Hertzian dipole in cylindrical coordinate in free space. In the following (178) will be used as reference model for the computation of the $z$ component of (102) in free space due to the $z$-oriented Hertzian dipole whereas the considerations remain unchanged for $\varphi$ and $\rho$-oriented electrical dipoles and more in general $u, v, w$ orthogonal-oriented dipoles.

\subsubsection{Numerical results Results}

In this Section the numerical solution for computing the SPD component of the dyadic Green's function (160) will be compared, in the near field region, with its equivalent analytical model in free space described in the previous 


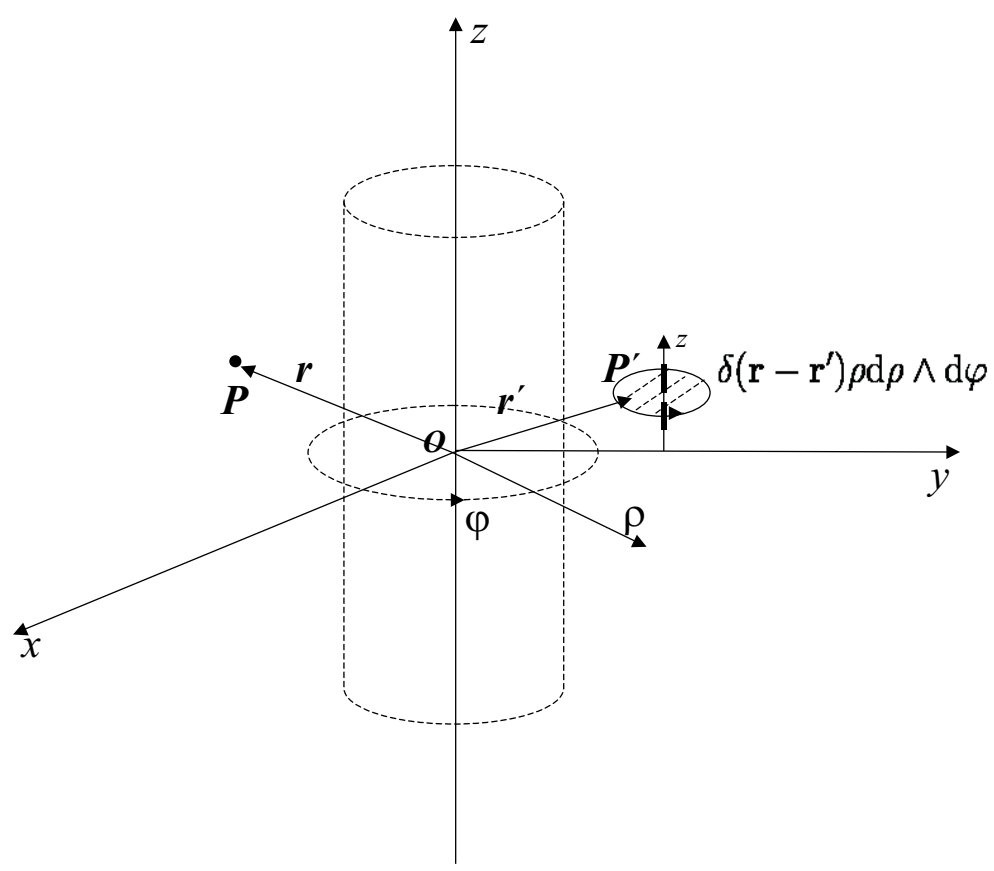

Figure 29.: The z-oriented Hertzian dipole at point $P^{\prime}=(1,0,1) \mathrm{m}$. 
Section in (179). We consider a $z$-oriented Hertzian dipole in free space placed at point $P^{\prime}=(1,0,1) \mathrm{m}$ of a circular cylindrical coordinate system as depicted in Figure 29.

To verify the the convergence and the correctness of the spatial domain Green's function (121) in the near field, we investigate the behavior of the $G_{z z}\left(\mathbf{r}, \mathbf{r}^{\prime}\right)$ in the source region. Therefore we reduce the observation to the $\varepsilon$ environment i.e. $\left|\mathbf{r}-\mathbf{r}^{\prime}\right|<\varepsilon$. As it has been explained in Section 7.3.1, the Green's function $\mathcal{G}_{e}\left(\mathbf{r}, \mathbf{r}^{\prime}\right)$ is not defined in $\mathbf{r}=\mathbf{r}^{\prime}$ where has a singularity of the order $1 / \mathbf{r}^{3}$. Analytically the value of the field in the source region can be computed introducing the distribution $\mathcal{G}_{e}^{i}\left(\mathbf{r}, \mathbf{r}^{\prime}\right)$ which represents the value of the field in the volume containing the source $V^{\prime}$.

Therefore, the analytical reference model (179) can be complemented in the source region by the distribution $\mathcal{G}_{e}^{i}\left(\mathbf{r}, \mathbf{r}^{\prime}\right)$. On the other hands, the use of this model for reference purposes in numerical computation can be problematic due to the definition of the $\varepsilon$ region. To verify this effect, we define the near field of the $z$-oriented Hertzian dipole assuming that the effective length dipole is $l=\lambda / 50$. In this case the Fresnel near field region [28] can be defined $0.62 \sqrt{l^{3} / \lambda}<\varepsilon<2 l^{2} / \lambda$ and therefore $8 e^{-4} \lambda<$ $\varepsilon<1.8 e^{-3} \lambda$.

We sample the field along $\rho$ coordinate letting constant $\varphi=0$ and $z=$ $1.001 \mathrm{~m}$. Defining the distance over $\lambda$ from the Hertzian dipole in $\rho$ direction as

$$
\frac{d}{\lambda}=\frac{\rho-\rho^{\prime}}{\lambda}
$$

We observe the field in the region $0.001<d / \lambda<4$, Figure 30. Therefore we observe the field in the near field inside the $\varepsilon$ region and outside. We set a relatively high number of angular mode $n=100$. From the Figure 32 we can see that the singularity of the Hertzian dipole appears already outside the $\delta-\varepsilon$, Figure 31 .

On the other hands the analytical value assigned from the distribution does not make sense outside the source region i.e. $\left|\mathbf{r}-\mathbf{r}^{\prime}\right|>\varepsilon$. The usage of the theory of distribution would require the implementation of the limit $\varepsilon \rightarrow 0$ to obtain the principal value of the integral which is a numerically sensitive to errors [41].

Therefore, we can conclude that such a model cannot be used as refer- 


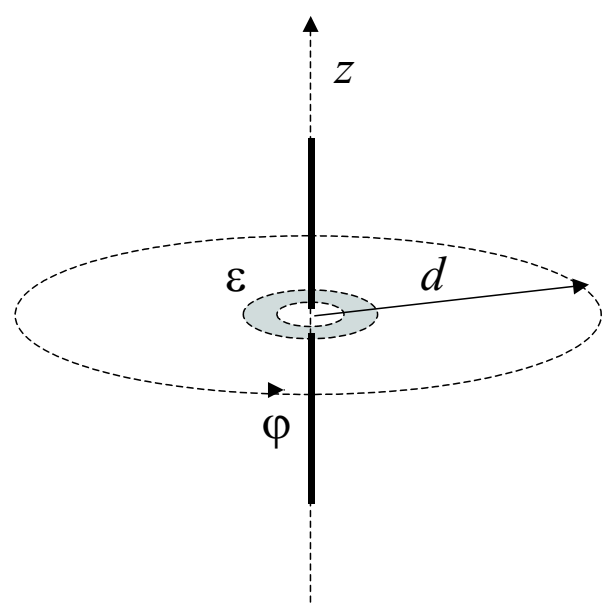

Figure 30.: Observation region over $\rho$.

ence model in the near field since the singularity cannot be described. On the other hands a good convergence can be observed outside the $\varepsilon$ region where conversely the model may be used correctly as reference. Before to introduce a more sophisticated model for the source region, an analysis of the numerical convergence properties outside the region the will be shown outside the source region where the effect of the singularity of the Hertzian dipole is not relevant i.e. $\left|\mathbf{r}-\mathbf{r}^{\prime}\right|>\delta-\varepsilon$. In particular, we investigate the convergence over $\varphi$ letting $\rho$ and $z$ constant and in the second case the convergence over $z$ letting $\varphi$ and $\rho$ constant. In the first case the observation region is taken for $z=1.001 \mathrm{~m}$ and $\rho=1.0025 \mathrm{~m}$ and moving around $\varphi$ form 0 to 180 at $\lambda=0.1 \mathrm{~m}$. The analysis is performed for three different values of the angular modes $n=60, n=80$ and $n=120$. Form Figures 33, 34 we can conclude that the SDP component of the Green's function computed via (121) shows a substantially convergence stability above 80 angular mode around the angle $\varphi$ while a low relative error is observed. In the second case in analogy with the $\rho$ case, we define a distance over lambda as follows

$$
\frac{d}{\lambda}=\frac{z-z^{\prime}}{\lambda}
$$




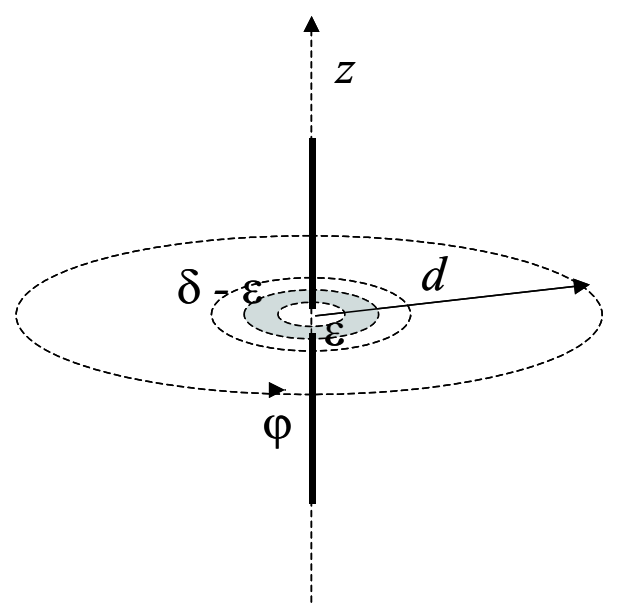

Figure 31.: The region $\delta-\varepsilon$ indicates the region in which the effect of the singularity becomes strong.

where the observation region is given for $0.0025<d / \lambda<1.4$ with $\varphi=0$, $\rho=1.0025 \mathrm{~m}$. Also in this case the convergence is substantially stable over $n=80$ angular mode while a relatively low relative error can be observed.

\subsubsection{Near Field of a Conformal Cylindrical Radiating Area}

In this Section an alternative reference model for near field computation will be introduced. In the second part the model will be used as reference model for convergence analysis of (121).

\subsubsection{Model Description}

The idea is that instead of using an infinitesimal Hertzian dipole as reference model, we use a small conformal sheet radiating area, Figure 35. We assume that the surface of the sheet $l A<<\lambda$. In this case for small distances form the sheet $\left|\mathbf{r}-\mathbf{r}^{\prime}\right|<\varepsilon$, the scalar Green's function $G\left(\mathbf{r}, \mathbf{r}^{\prime}\right)$ can be expanded 


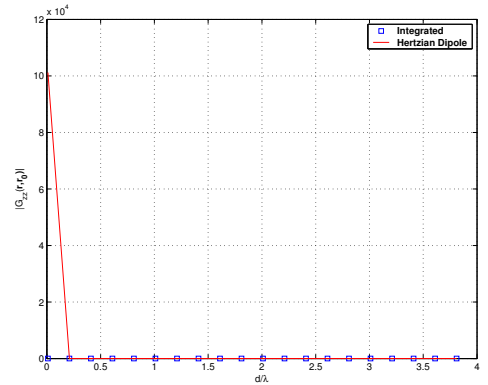

(i) $\left|G_{z z}\left(\mathbf{r}, \mathbf{r}^{\prime}\right)\right|$ over $\rho$ for $n=120$

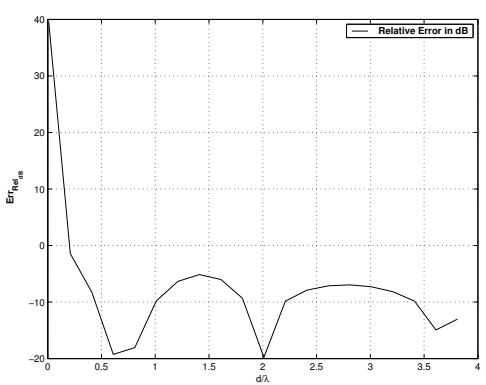

(ii) Relative error over $\rho$ in $d B$ for $n=120$

Figure 32.: Comparison between the $\left|G_{z z}\left(\mathbf{r}, \mathbf{r}^{\prime}\right)\right|$ observed along $\rho$ direction inside and outside the $\epsilon$ region and the $E_{z}$ component of the Hertzian dipole (178). The number angular modes $n=120$ and $\lambda=0.1 \mathrm{~m}$.

into a power series expansions as follows

$$
G\left(\mathbf{r}, \mathbf{r}^{\prime}\right)=\frac{e^{-j k\left|\mathbf{r}-\mathbf{r}^{\prime}\right|}}{4 \pi \epsilon\left|\mathbf{r}-\mathbf{r}^{\prime}\right|}=\frac{1}{4 \pi \epsilon}\left(\frac{1}{\left|\mathbf{r}-\mathbf{r}^{\prime}\right|}-j k-\frac{1}{2} k^{2}\left|\mathbf{r}-\mathbf{r}^{\prime}\right|+\ldots\right)
$$

therefore in this region, the singular behavior of the Green's function is equivalent to static case

$$
G\left(\mathbf{r}, \mathbf{r}^{\prime}\right) \simeq G_{0}\left(\mathbf{r}, \mathbf{r}^{\prime}\right)=\frac{1}{4 \pi \epsilon\left|\mathbf{r}-\mathbf{r}^{\prime}\right|}
$$

In this case the electric field may be derived from the scalar potential form

$$
\mathcal{E}=-\mathrm{d} \psi_{\mathcal{A}}
$$

the scalar potential form is related to the scalar Green's function by means of the Coulomb integral equation

$$
\psi_{\mathcal{A}}(\mathbf{r})=\int_{V^{\prime}} \frac{\mathcal{Q}^{\prime}}{4 \pi \epsilon\left|\mathbf{r}-\mathbf{r}^{\prime}\right|}
$$

where $\mathcal{Q}^{\prime}$ is the charge distribution form given as follows

$$
\mathcal{Q}^{\prime}\left(\mathbf{r}^{\prime}\right)=q^{\prime} \rho^{\prime} \mathrm{d} \rho^{\prime} \wedge \mathrm{d} \varphi^{\prime} \wedge \mathrm{d} z^{\prime}
$$




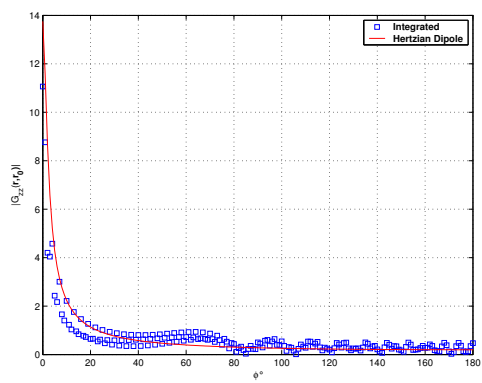

(i) $\left|G_{z z}\right|$ over $\varphi$ for $n=60$.

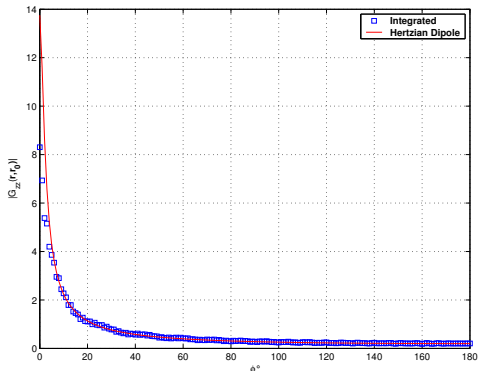

(iii) $\left|G_{z z}\right|$ over $\varphi$ for $n=80$.

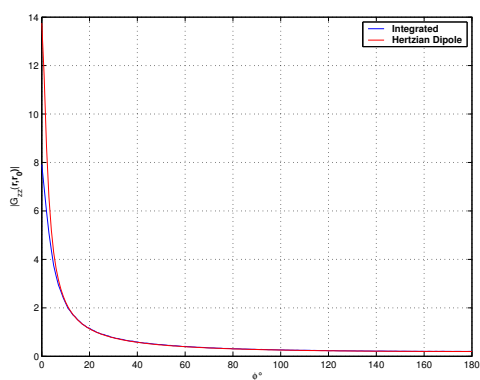

(v) $\left|G_{z z}\right|$ over $\varphi$ for $n=120$.

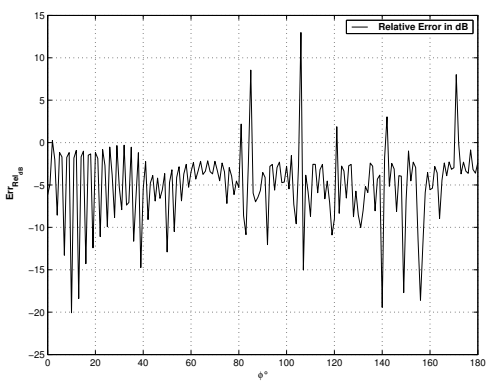

(ii) Relative error over $\varphi$ in $d B$ for for $n=$ 60 .

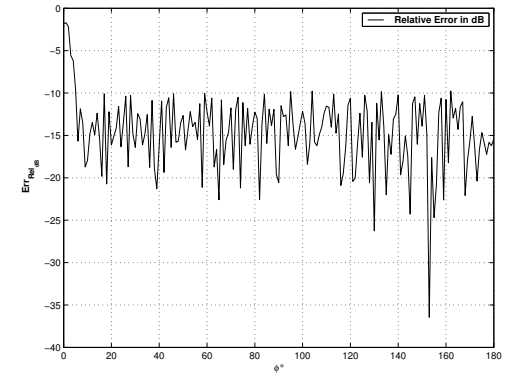

(iv) Relative error over $\varphi$ in $d B$ for for $n=$ 80.

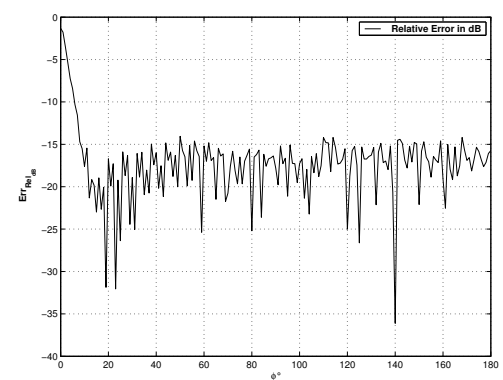

(vi) Relative error over $\varphi$ in $d B$ for for $n=$ 120.

Figure 33.: Comparison between the $\left|G_{z z}\left(\mathbf{r}, \mathbf{r}^{\prime}\right)\right|$ observed around $\varphi$ direction and the $E_{z}$ component of the Hertzian dipole, (178). In this case the the field is sampled outside the singular region at $(d / \lambda=0.03)$. The number angular modes $n=60, n=80, n=120$ and $\lambda=0.1 \mathrm{~m}$. 


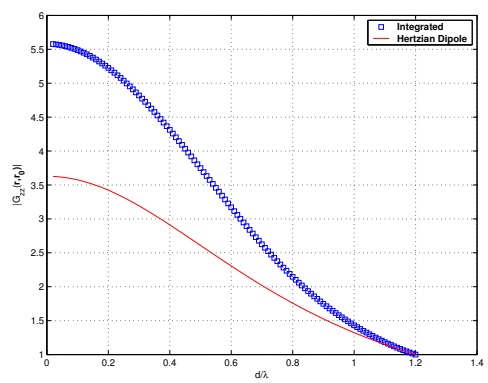

(i) $\left|G_{z z}\right|$ over $z$ for $n=60$.

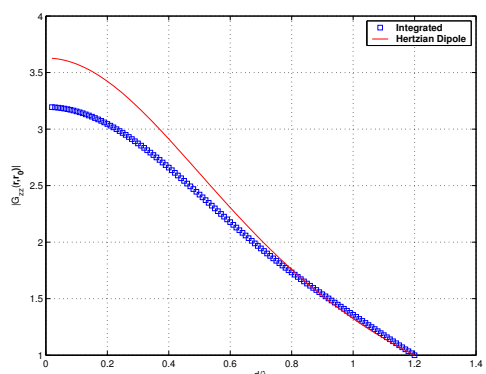

(iii) $\left|G_{z z}\right|$ over $z$ for $n=80$.

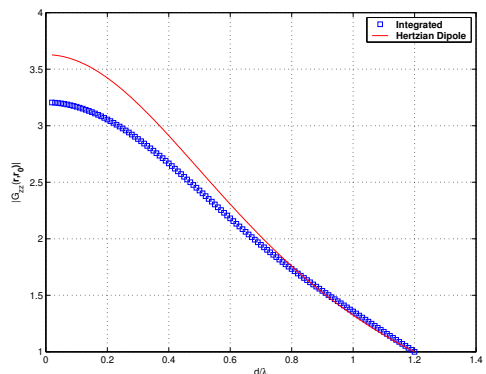

(v) $\left|G_{z z}\right|$ over $z$ for $n=120$.

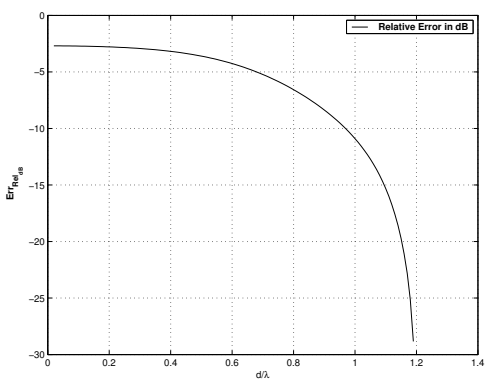

(ii) Relative error over $z$ in $d B$ for $n=60$.

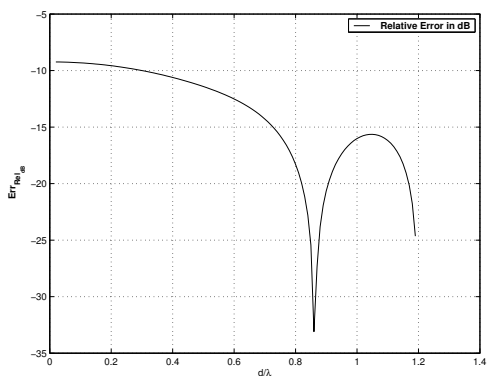

(iv) Relative error over $z$ in $d B$ for $n=80$.

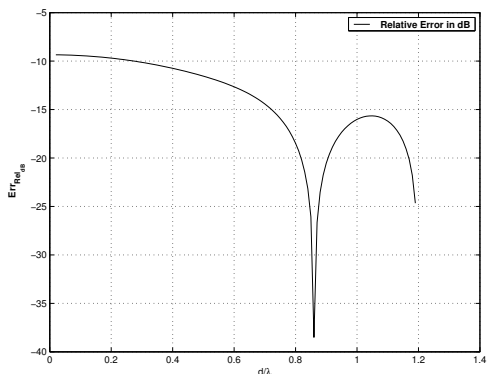

(vi) Relative error over $z$ in $d B$ for $n=120$.

Figure 34.: Comparison between the $\left|G_{z z}\left(\mathbf{r}, \mathbf{r}^{\prime}\right)\right|$ observed along $z$ direction and the $E_{z}$ component of the Hertzian dipole, (178). In this case the field is sampled outside the singular region at $(d / \lambda=0.03)$. The number angular modes $n=60, n=80, n=120$ and $\lambda=0.1 \mathrm{~m}$. 


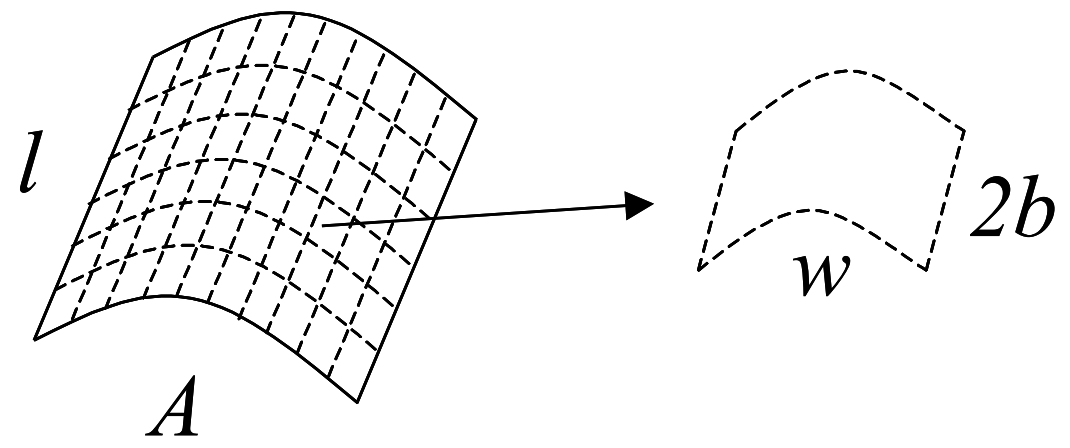

Figure 35.: Cylindrical Conformal Radiating Sheet of Current.

As the volume of the source contracts to a surface, the contribute of the radial component of the charge in $\rho$ and the from the end of the sheet becomes varnishing small. Therefore the charge density is distributed on the surface

$$
\left.\mathcal{Q}_{A}^{\prime}=\mathrm{d} \rho^{\prime}\right\lrcorner \mathcal{Q}
$$

In this case, the Coulomb integral equation is transformed to two dimensional integral equation as follows

$$
\left.\psi_{\mathcal{A}}(\mathbf{r})=\mathrm{d} \rho^{\prime}\right\lrcorner\left(\int_{V^{\prime}} \frac{\mathcal{Q}^{\prime}}{4 \pi \epsilon\left|\mathbf{r}-\mathbf{r}^{\prime}\right|}\right)=\int_{S^{\prime}} \frac{\mathcal{Q}_{A}^{\prime}}{4 \pi \epsilon\left|\mathbf{r}-\mathbf{r}^{\prime}\right|}
$$

where $\mathcal{Q}_{A}^{\prime}$ is unknown. In the following a method of moment (MOM) algorithm to solve the integral equation (189) to compute the unknown charge distribution will be described.

We are consider an ideal metal surface. Therefore the surface is equipotential. That means that the value of the potential over the surface of the metal is constant and unknown. More explicitly we can rewrite (189) for the conformal sheet of current in the following way

$$
\int_{\varphi^{\prime}-\frac{A}{2 \rho^{\prime}}}^{\varphi^{\prime}+\frac{A}{2 \rho^{\prime}}} \int_{z^{\prime}-\frac{l}{2}}^{z^{\prime}+\frac{l}{2}} \frac{1}{4 \pi \epsilon} \frac{\mathcal{Q}_{A}^{\prime}\left(\rho^{\prime}, \varphi^{\prime}\right)}{\sqrt{\rho^{\prime 2}\left(\varphi-\varphi^{\prime}\right)^{2}+z^{\prime 2}}} \rho^{\prime} \mathrm{d} \varphi^{\prime} \mathrm{d} \rho^{\prime}=\psi_{\mathcal{A}_{0}}\left(\rho^{\prime}, \varphi^{\prime}\right)=\text { const }
$$


where the surface charge $\mathcal{Q}_{A}^{\prime}$ is unknown. The (190) may be solved via the MOM [52]. Since the dimensions of the sheet are small compared with the wavelength, it is reasonable assume the charge concentrated at the center of each subsection and expand the charge distribution in the following set of basis functions

$$
\begin{array}{r}
\mathcal{Q}_{A}^{\prime}\left(\varphi^{\prime}, z^{\prime}\right)=\sum_{n=1}^{N_{s}} a_{n} \Phi_{n} \\
\Phi_{n}=\delta\left(\varphi-\varphi_{n}^{\prime}\right) \delta\left(z-z_{n}^{\prime}\right)
\end{array}
$$

Inserting (191) into the left side of (190) we obtain

$$
\sum_{n=1}^{N_{s}} a_{n} \int_{\varphi^{\prime}-\frac{A}{2 \rho^{\prime}}}^{\varphi^{\prime}+\frac{A}{2 \rho^{\prime}}} \int_{z^{\prime}-\frac{l}{2}}^{z^{\prime}+\frac{l}{2}} \frac{1}{4 \pi \epsilon} \frac{\sigma_{A} \delta\left(\varphi-\varphi_{n}^{\prime}\right) \delta\left(z-z_{n}^{\prime}\right)}{\sqrt{\rho^{\prime 2}\left(\varphi-\varphi^{\prime}\right)^{2}+z^{\prime 2}}} \rho^{\prime} \mathrm{d} \varphi^{\prime} \mathrm{d} \rho^{\prime}
$$

We choose as test function also Dirac (i.e. Galerkin method) functions to define our inner product and we obtain for $m \neq n$

$$
\begin{array}{r}
Z_{m n}=\sum_{m=1}^{N_{t}} \sum_{n=1}^{N_{s}} a_{n} \int_{S} \delta\left(\varphi-\varphi_{m}\right) \delta\left(z-z_{n}\right) \\
\left(\int_{S^{\prime}} \frac{1}{4 \pi \epsilon} \frac{\delta\left(\varphi-\varphi_{n}^{\prime}\right) \delta\left(z-z_{n}^{\prime}\right)}{\sqrt{\rho^{\prime 2}\left(\varphi-\varphi^{\prime}\right)^{2}+z^{\prime 2}}} \rho^{\prime} \mathrm{d} \varphi^{\prime} \mathrm{d} \rho^{\prime}\right) \rho \mathrm{d} \varphi \mathrm{d} \rho
\end{array}
$$

where we choose the number of basis functions $\left(N_{s}\right)$ equal the number of test functions $\left(N_{t}\right)$. Also the we must define the inner product for the left side of (189) we obtain

$\beta_{m}=\int_{S} \delta\left(\varphi-\varphi_{m}\right) \delta\left(z-z_{m}\right) \psi_{\mathcal{A} 0}(\varphi, z)=\psi_{\mathcal{A} 0}\left(\varphi_{m}, z_{m}\right)=\psi_{\mathcal{A} 0} \quad m=1, \ldots, N_{t}$

The case in which $m=n$ describes the interaction of the charge with itself and must be treated separately since is singular $\left(\left|\mathbf{r}_{m}-\mathbf{r}_{m}\right|=0\right)$. The self interaction can be modelled in the usual way integrating in the subsection surface as following

$$
\begin{array}{r}
Z_{m m}=\int_{-w / 2 \rho^{\prime}}^{w / 2 \rho^{\prime}} \mathrm{d} \varphi^{\prime} \int_{-b}^{b} \frac{1}{4 \pi \epsilon} \frac{a_{m}}{\sqrt{\rho^{\prime 2}\left(\varphi-\varphi_{m}^{\prime}\right)^{2}+z_{m}^{\prime 2}}} \mathrm{~d} z^{\prime}= \\
w \ln \left(b+\sqrt{\rho^{\prime 2}+b^{2}}\right)-w \ln \left(-b+\sqrt{\rho^{\prime 2}+b^{2}}\right)
\end{array}
$$




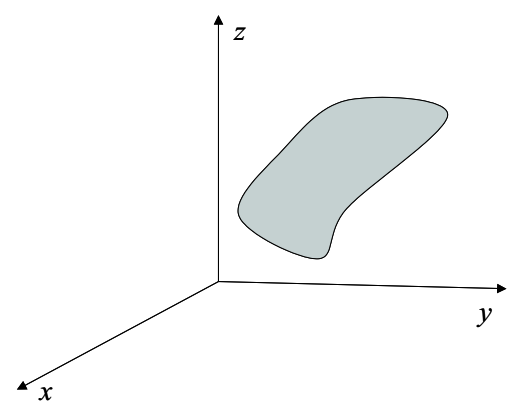

Figure 36.: Arbitrary small sheet radiating surface.

Therefore we transformed (190) in a linear system of equation that can be summarized in matrix notation as follows

$$
Z_{m n} a_{n}=\beta_{m}
$$

The solution of (196) gives an accurate description of the charge distribution. Having computed the charge distribution the electric field form can be computed in the usual way as follows

$$
\mathcal{E}=-\mathrm{d} \psi_{A}
$$

The (197) will be used as reference model for the near field computation. The result can be easily extended for any other geometry of the surface that can be described by an orthogonal system of coordinate, Figure 36

$$
\psi_{\mathcal{A}}(u, v)=\int_{S^{\prime}} \frac{1}{4 \pi \varepsilon} \frac{\mathcal{Q}_{S}^{\prime}(u, v)}{\sqrt{s_{1}^{2}+s_{2}^{2}}}
$$

where $s_{1}=g_{1} \mathrm{~d} u$ and $s_{2}=g_{2} \mathrm{~d} v$.

\subsubsection{Numerical Results for the Conformal Radiating Sheet Area.}

In this section the same set of simulations of Section 7.3 .5 are repeated but using for the radiating conformal sheet. We may note that such reference 


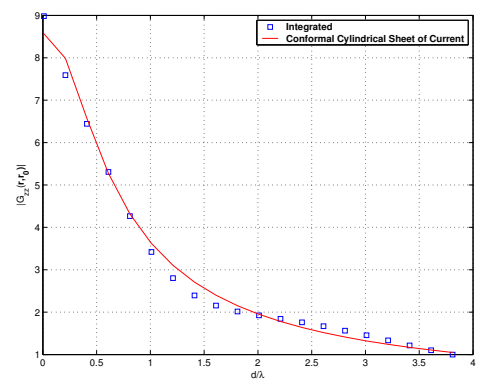

(i) $\left|G_{z z}\right|$ over $\rho$ for $n=80$.

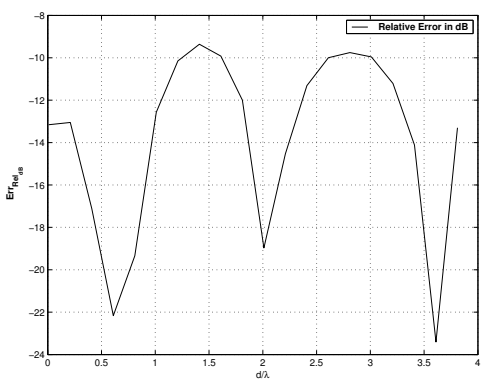

(ii) $\left|G_{z z}\right|$ over $\rho$ for $n=80$.

Figure 37.: Comparison between the $\left|G_{z z}\left(\mathbf{r}, \mathbf{r}^{\prime}\right)\right|$ observed along $\rho$ direction inside and outside the $\epsilon$ region and the $E_{z}$ component of the conformal radiating sheet area. The number angular modes $n=80$ and $\lambda=0.1 \mathrm{~m}$ and the minimum distance $(d / \lambda=0.001)$.

model can serve also as reference model for the MOM computation when subsection technique is used. In fact each subsection can be modelled as well as the small radiating conformal sheet in the near field region in the same way. 


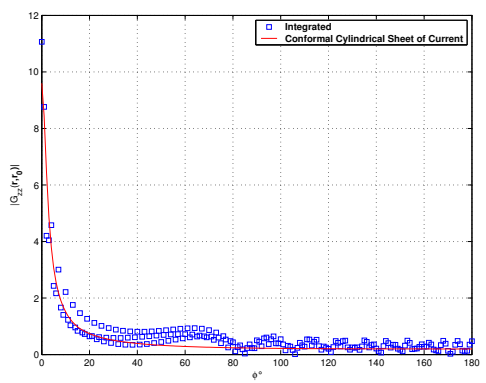

(i) $\left|G_{z z}\right|$ over $\varphi$ for $n=60$.

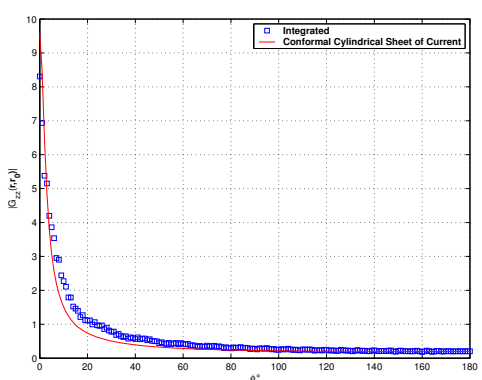

(iii) $\left|G_{z z}\right|$ over $\varphi$ for $n=80$.

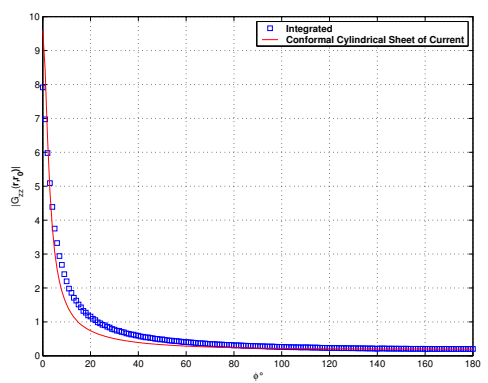

(v) $\left|G_{z z}\right|$ over $\varphi$ for $n=120$.

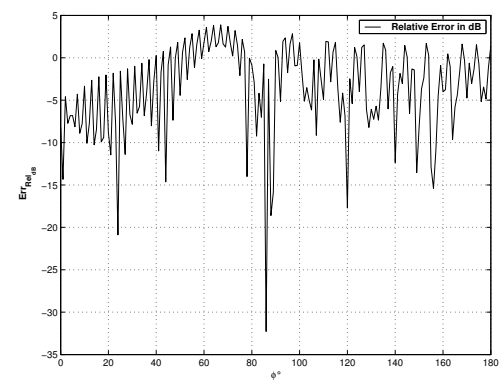

(ii) Relative error over $\varphi$ in $d B$ for $n=60$.

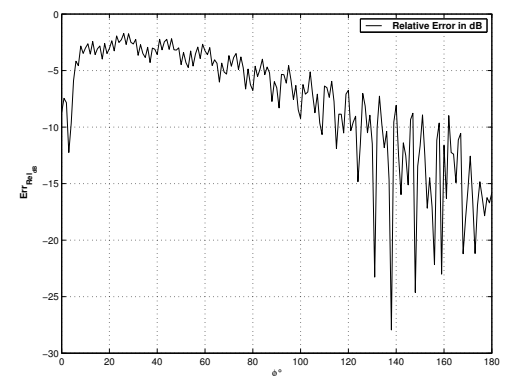

(iv) Relative error over $\varphi$ in $d B$ for $n=80$.

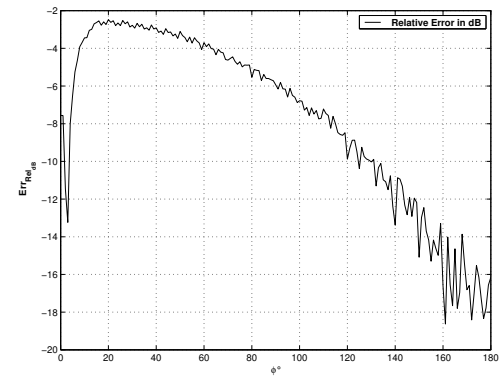

(vi) Relative error over $\varphi$ in $d B$ for $n=120$.

Figure 38.: Comparison between the $\left|G_{z z}\left(\mathbf{r}, \mathbf{r}^{\prime}\right)\right|$ observed around $\varphi$ direction and the $E_{z}$ component of the conformal sheet radiating area. In this case the the field is sampled at $(d / \lambda=0.001)$. The number angular modes $n=60, n=80$, $n=120$ and $\lambda=0.1 \mathrm{~m}$. 


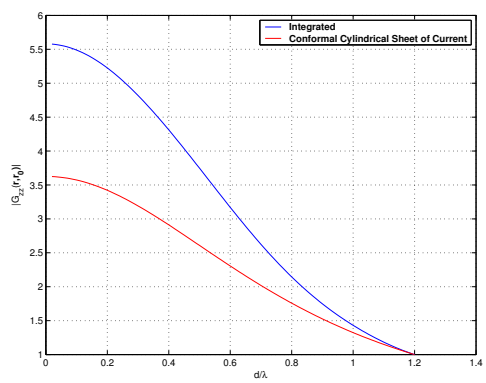

(i) $\left|G_{z z}\right|$ over $z$ for $n=60$.

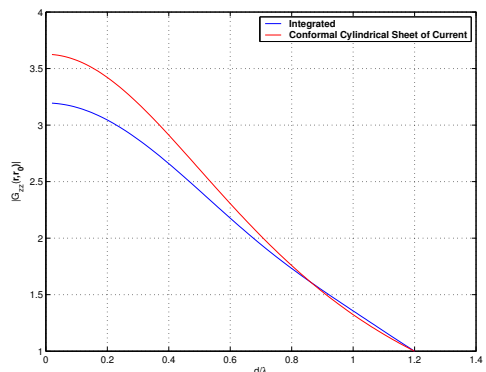

(iii) $\left|G_{z z}\right|$ over $z$ for $n=80$.

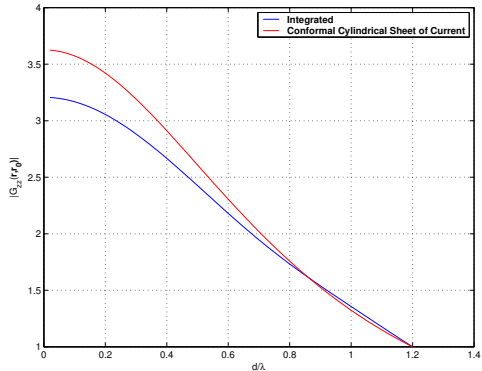

(v) $\left|G_{z z}\right|$ over $z$ for $n=120$.

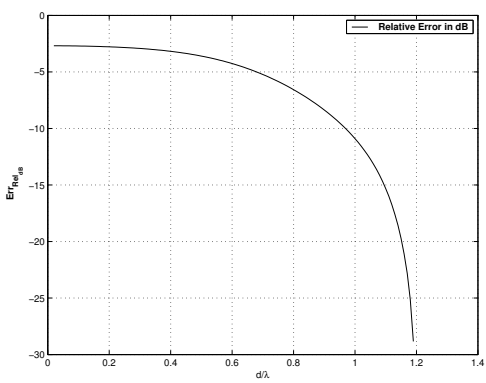

(ii) Relative error over $z$ in $d B$ for $n=60$.

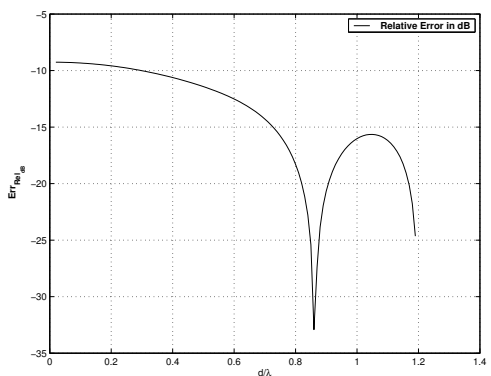

(iv) Relative error over $z$ in $d B$ for $n=80$.

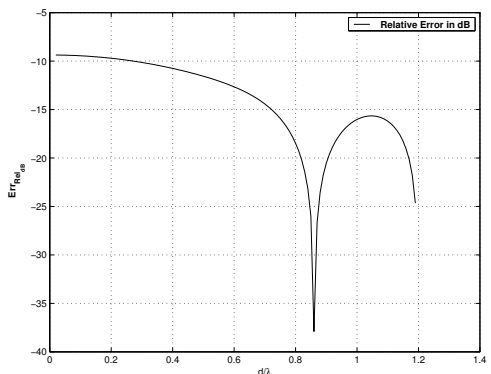

(vi) $\left|G_{z z}\right|$ Relative error over $z$ in $d B$ for $n=120$.

Figure 39.: Comparison between the $\left|G_{z z}\left(\mathbf{r}, \mathbf{r}^{\prime}\right)\right|$ observed around $z$ direction and the $E_{z}$ component of the conformal sheet radiating area. In this case the the field is sampled at $(d / \lambda=0.001)$. The number angular modes $n=60, n=80$, $n=120$ and $\lambda=0.1 \mathrm{~m}$. 


\section{Application and Validation}

In the following Chapter, the cylindrical Green's function is applied with MoM. In Section 8.1 the MoM formulation in SD in is given. In Section 8.2 the application to a radome problem is given and comparison with measurements are presented. Finally in Section 8.3 the performance of the IEM in terms of computation time and memory allocation are presented and discussed.

\subsection{Moment Method Formulation}

We rewrite the field solution for the electric field form $\mathcal{E}$ in its integral equation form [21]

$$
\mathcal{E}(\mathbf{r})=\int_{V^{\prime}} \mathcal{G}_{e}^{i j}\left(\mathbf{r}, \mathbf{r}^{\prime}\right) \wedge \mathcal{J}^{j}\left(\mathbf{r}^{\prime}\right)
$$

where $\mathcal{G}_{e}^{i j}\left(\mathbf{r}, \mathbf{r}^{\prime}\right)$ is the dyadic Green's function, $\mathcal{J}^{j}\left(\mathbf{r}^{\prime}\right)$ is the current density distribution, $\mathbf{r}$ and $\mathbf{r}^{\prime}$ are the observation and source coordinate, $V^{\prime}$ the volume containing the sources, $i$ and $j$ are the observation and source layer. Considering that the radiating element are sheets of current, the current density $\mathcal{J}^{j}\left(\mathbf{r}^{\prime}\right)$ is a surface current density expressed by [21]

$$
\mathcal{J}^{j}\left(\mathbf{r}^{\prime}\right)=\delta\left(\rho-\rho^{\prime}\right) \mathrm{d} \rho \wedge \mathcal{J}_{S^{\prime}}^{j}\left(\rho^{\prime}, \varphi^{\prime}, z^{\prime}\right)
$$

where $\mathcal{J}_{S^{\prime}}^{j}\left(\varphi^{\prime}, z^{\prime}\right)$ represents the current density form with components in $\varphi$ and $z$ directions and $S^{\prime}$ surface of the sources. Inserting (200) into (199) we obtain

$$
\mathcal{E}(\mathbf{r})=\int_{S^{\prime}} \mathcal{G}_{e}^{i j}\left(\mathbf{r}, \mathbf{r}^{\prime}\right) \wedge \mathcal{J}_{S^{\prime}}^{j}\left(\mathbf{r}^{\prime}\right)
$$

For the MOM definition, it is of our interest to extract the tangential component of the electric field. That can be done by eliminating the radial 
dependency as follows

$$
\left.\mathcal{E}_{t}(\mathbf{r})=\int_{S^{\prime}} \mathrm{d} \rho\right\lrcorner\left(\mathrm{d} \rho \wedge \mathcal{G}_{e}\left(\mathbf{r}, \mathbf{r}^{\prime}\right)\right) \wedge \mathcal{J}_{S^{\prime}}\left(\mathbf{r}^{\prime}\right)
$$

Similarly to the Chapter 6 , considering the cylindrical symmetry, we expand (202) in cylindrical waves and obtain

$$
\mathcal{E}_{t}(\mathbf{r})=\sum_{n=1}^{+\infty} e^{-j n\left(\phi-\phi^{\prime}\right)} \int_{k_{z}} \overline{\mathcal{G}}_{t}\left(\rho, \rho^{\prime}, k_{z}, n\right)\left[\begin{array}{c}
J_{\phi_{n}} \\
J_{k_{z}}
\end{array}\right] e^{-j k_{z}\left(z-z^{\prime}\right) \mathrm{d} k_{z}}
$$

where $\overline{\mathcal{G}}_{t}\left(\rho, \rho^{\prime}, k_{z}, n\right)$ is the spectral domain representation of the dyadic double one form

$$
\overline{\mathcal{G}}_{t}\left(\rho, \rho^{\prime}, k_{z}, n\right)=\left[\begin{array}{ll}
\bar{G}_{\phi \phi^{\prime}} & \bar{G}_{\phi z^{\prime}} \\
\bar{G}_{z \phi^{\prime}} & \bar{G}_{z z^{\prime}}
\end{array}\right]
$$

which in spectral domain representation is related with the tangential electric field as follows

$$
\left[\begin{array}{c}
E_{\phi_{n}} \\
E_{z_{n}}
\end{array}\right]=\left[\begin{array}{ll}
\bar{G}_{\phi \phi^{\prime}} & \bar{G}_{\phi z^{\prime}} \\
\bar{G}_{z \phi^{\prime}} & \bar{G}_{z z^{\prime}}
\end{array}\right]\left[\begin{array}{c}
J_{\phi_{n}} \\
J_{k_{z}}
\end{array}\right]
$$

To define the MOM matrix representation we observe that over the metallic surface of the radiating elements, we can expand the tangential component of the electric and magnetic field form into a set on orthonormal basic functions at $\rho=\rho^{\prime}$

$$
\begin{aligned}
& \mathcal{E}_{t}(\mathbf{r})=\sum_{l=1}^{N_{b}} V_{n}^{p} e_{l}\left(\varphi^{\prime}, z^{\prime}\right) \\
& \mathcal{H}_{t}(\mathbf{r})=\sum_{l=1}^{N_{b}} I_{n}^{p} h_{l}\left(\varphi^{\prime}, z^{\prime}\right)
\end{aligned}
$$

where $p=\varphi, z, N_{b}$ is the number of basis functions, $e_{l}\left(\varphi^{\prime}, z^{\prime}\right)$ and $h_{l}\left(\varphi^{\prime}, z^{\prime}\right)$ are the basis structure functions for the electric and magnetic fields. We assume to decompose the radiating surface element $\varphi^{\prime}, z^{\prime}$ in subsection to which a given basic function is assigned. We also consider the structure functions to be Fourier transformable and that can factorized in $\varphi^{\prime}$ and $z^{\prime}$ in the following way

$$
\begin{aligned}
e_{l}\left(\varphi^{\prime}, z^{\prime}\right) & =e_{l}\left(\varphi^{\prime}\right) e_{l}\left(z^{\prime}\right) \\
h_{l}\left(\varphi^{\prime}, z^{\prime}\right) & =h_{l}\left(\varphi^{\prime}\right) h_{l}\left(z^{\prime}\right)
\end{aligned}
$$


We note that over the perfect metallic surface of the radiating elements the $\mathcal{J}_{S^{\prime}}\left(\mathbf{r}^{\prime}\right)=\mathcal{H}_{t}(\mathbf{r})$. Considering this and inserting (207) into (8.1) we obtain

$$
\begin{array}{r}
{\left[\begin{array}{c}
V_{l}^{\varphi} e_{l}\left(\varphi^{\prime}, z^{\prime}\right) \\
V_{l}^{z} e_{l}\left(\varphi^{\prime}, z^{\prime}\right)
\end{array}\right]=\sum_{n=1}^{+\infty} e^{-j n\left(\phi-\phi_{l}^{\prime}\right)}} \\
\int_{k_{z}} \overline{\mathcal{G}}_{t}\left(\rho, \rho^{\prime}, k_{z}, n\right)\left[\begin{array}{c}
I_{l}^{\varphi} h_{l}(n) \\
-I_{l}^{z} h_{l}\left(k_{z}\right)
\end{array}\right] e^{-j k_{z}\left(z-z_{l}^{\prime}\right) \mathrm{d} k_{z}}
\end{array}
$$

(208)

where $I_{l}^{z}$ and $I_{l}^{\varphi}$ are the unknown complex current coefficients. Multiplying both sides of (209) for $e_{k}^{*}(\varphi, z)$ and moving in the observation coordinate in each subsection $l$ we obtain that

$$
\left[\begin{array}{c}
V_{l}^{\varphi} \\
V_{l}^{z}
\end{array}\right]=\mathbf{Z}_{l k}\left[\begin{array}{c}
I_{l}^{\varphi} \\
-I_{l}^{z}
\end{array}\right]
$$

where

$$
\mathbf{Z}_{l k}=\sum_{n=1}^{+\infty} e^{-j n\left(\phi_{k}-\phi_{l}^{\prime}\right)} \int_{k_{z}} \overline{\mathcal{G}}_{t}\left(\rho, \rho^{\prime}, k_{z}, n\right)\left[\begin{array}{c}
h_{l}(n) \\
h_{l}\left(k_{z}\right)
\end{array}\right] e^{-j k_{z}\left(z_{k}-z_{l}^{\prime}\right) \mathrm{d} k_{z}}
$$

The feeding point model will be specified in the next section when the modelled antenna geometry for the specific application will be described.

\subsection{Application and Validation}

In this section a conformal dipole antenna is investigated with the IEM introduced in this work. The dipoles is mounted around a conducting cylinder of height $300 \mathrm{~mm}$ and diameter $105 \mathrm{~mm}$. The dipole is embedded in a cylindrical radome, Figure 43. The radome and characteristics are reported in Table 1. The dipole width over the circumference is $w=0.01 \mathrm{~m}$ and the height is $L=\lambda / 2$ at $1.75 \mathrm{GHz}$. The gap width at the feeding point is 
Table 1.: Radome characteristics.

\begin{tabular}{lr}
\hline Radome material & Polyethylene $(\mathrm{PE})$ \\
Dielectric constant & $2.25(1.7-2 G H z)$ \\
Height & $300 \mathrm{~m} \mathrm{~m}$ \\
Inner Diameter & $28 \mathrm{~m} \mathrm{~m}$ \\
Outer Diameter & $29.2 \mathrm{~m} \mathrm{~m}$
\end{tabular}

$h=0.001 \mathrm{~m}$, Figure 40. Since $w / \lambda=0.0667$ the current can be considered to flow in axial direction. For the computation of the axial current distribution the MOM is applied in spectral domain using subsectional basis functions of rectangular shape.

\subsubsection{Modelling the effect of $\mathrm{w}$}

The influence of the $w$ is accounted by modelling the angular $\varphi$ dependence of the current in one subsection, Figure 40. We observe that the influence of $w$ is relevant for the input impendence computation and the radiation pattern. To choose the shape of the subsection modelling the effect of the current along $w$, we investigate the four different shapes. We assume that the current can be studied separately in $\varphi$ and $z$ and therefore $\mathcal{J}_{S^{\prime}}(\varphi, z)=$ $J(\varphi) J(z) \rho \mathrm{d} \rho \wedge \mathrm{d} \varphi$ and to be Fourier transformable. Therefore we can write at point $P=\left(\rho=\rho^{\prime}, \varphi, z\right)$

$$
\mathcal{J}_{S^{\prime}}(\varphi, z)=\rho \mathrm{d} \rho \wedge \mathrm{d} \varphi \sum_{n=0}^{+\infty} J_{\varphi}(n) e^{-j n\left(\varphi-\varphi^{\prime}\right)} \int_{k_{z}} J_{k_{z}} e^{-j k_{z}\left(z-z^{\prime}\right)} \mathrm{d} k_{z}
$$

For the $\varphi$ current we choose four different basis functions shapes: triangular (rooftop), rectangular, Dirac, and roll-off cosines. At $\rho^{\prime}=0.05 \mathrm{~m}$ and $z^{\prime}=0$ we investigate the convergence over $\varphi$ of the different basis functions for different number of angular modes, Figure 8.2.1. We can see that the lowest relative error over $\varphi$ is for the triangular basis functions already for $n=80$ angular modes and therefore we choose triangular shape for modelling in the current over $\varphi$, Figure 40 . 


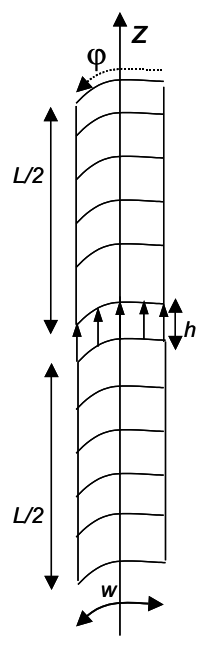

(i) Dipole geometry.

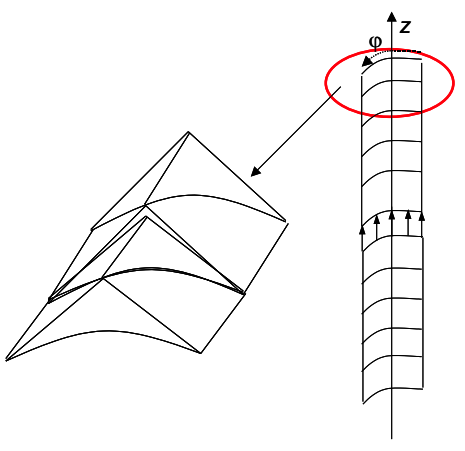

(ii) Dipole discretization.

Figure 40.: Dipole geometry and qualitative description of the dipole discretization.

\subsubsection{Antenna Feeding}

The used excitation type in the antenna is a forced voltage $V_{g}$ in the gap $h$. The associate electric field form is $\mathcal{E}^{i}=E_{g} \mathrm{~d} z$ such that in the gap

$$
V_{g}=-\int_{-h / 2}^{h / 2} \mathcal{E}^{i}=-h E_{g}
$$

In this case the boundary conditions for the electric field impose that $E_{z}=0$ on the dipole arms and $E_{z}=E_{g}$ in the feeding gap. The current density is solved transforming the integral equation (102) into a linear system of equations.

\subsubsection{Measurement Results}

The comparison with the measurement is first performed in terms of Return Loss for the dipole in free space. For the fabricated antenna the used 


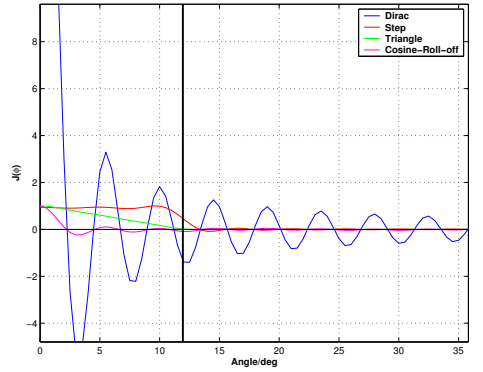

(i) Current density responses for for $n=80$.

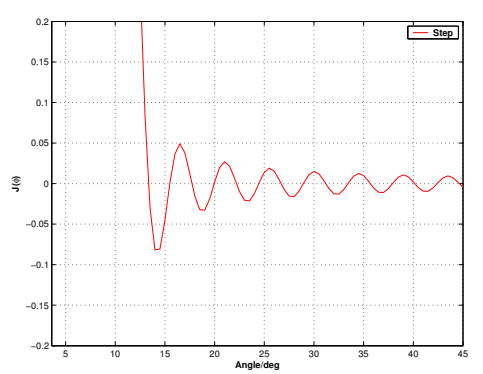

(iii) Residual error current for stepfunction over $\varphi$ for $n=80$.

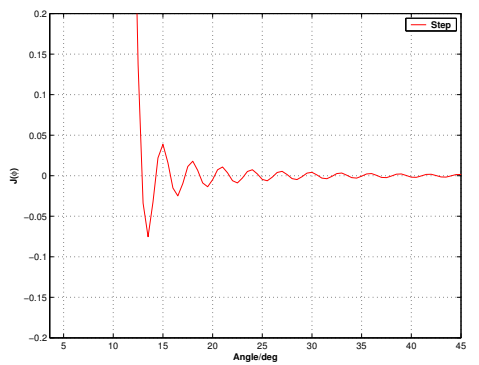

(v) Residual error current for stepfunction over $\varphi$ for $n=120$.

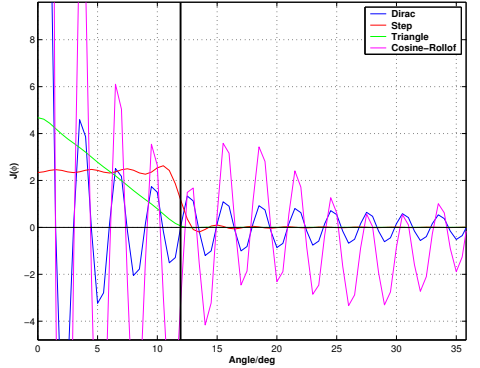

(ii) Current density responses for for $n=120$.

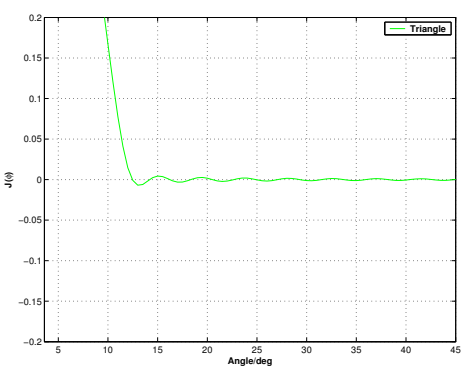

(iv) Residual error current for triangular over $\varphi$ for $n=80$.

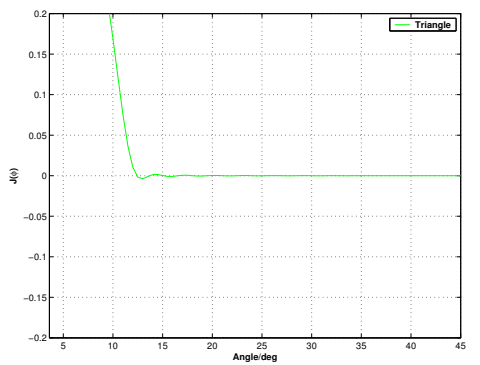

(vi) Residual error current for triangular over $\varphi$ for $n=120$.

Figure 41.: Convergence analysis of different shape of the basis functions over $\varphi$ for $n=$ 80,120 . 


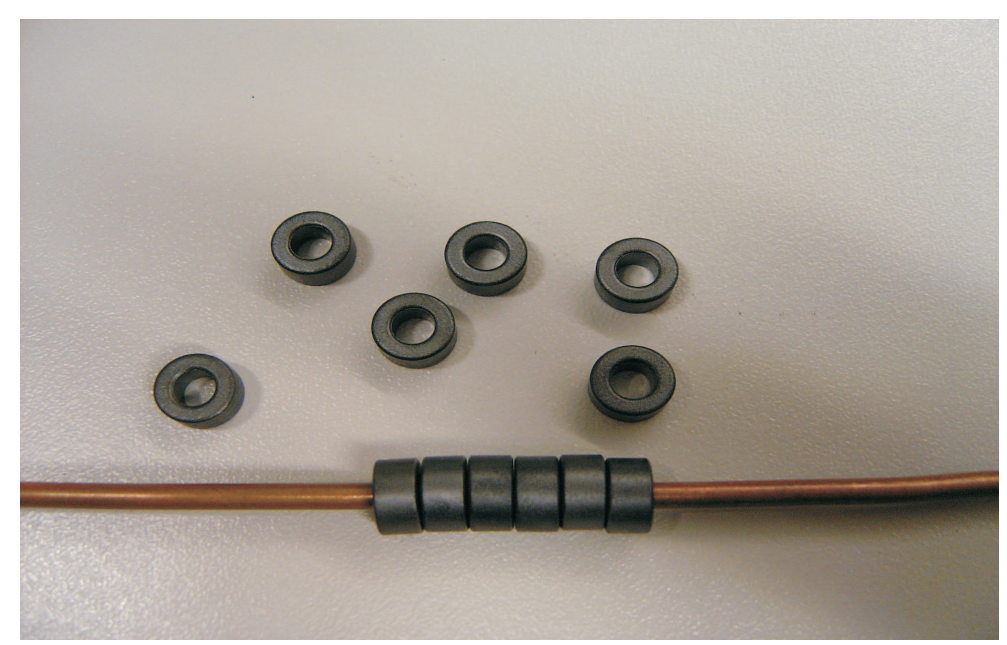

Figure 42.: Coaxially loaded ferrite balun.

balun is a ferrite loaded coaxial balun, Figure 42. The comparison between measurements and simulation using the IEM are shown in Figure 44 and good agreement can be observed. The comparison is then performed vertical radiation pattern (VP) and horizontal radiation pattern (HP) of the antenna in two different configuration. In the first case the VP and HP are compared to the measured ones with only the cylindrical reflector and the dipole, Figure 43i. In the second case the VP and HP are compared with the measured at the previous configuration is embedded into a one layer radome, Figure 43ii. In both cases the comparison is performed at $1.75 \mathrm{GHz}$. Also in these cases a good agreement with the measurement is shown, Figures 45i, 45ii, 45iii and 45iv, 

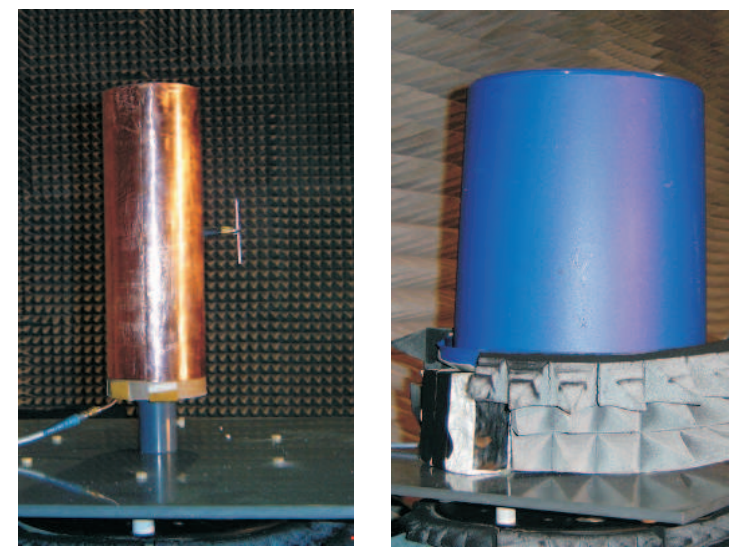

(i) Conformal dipole antenna and cylindrical reflector.

(ii) Conformal dipole antenna and cylindrical reflector embedded into cylindrical radome.

Figure 43.: Measurement setup. 


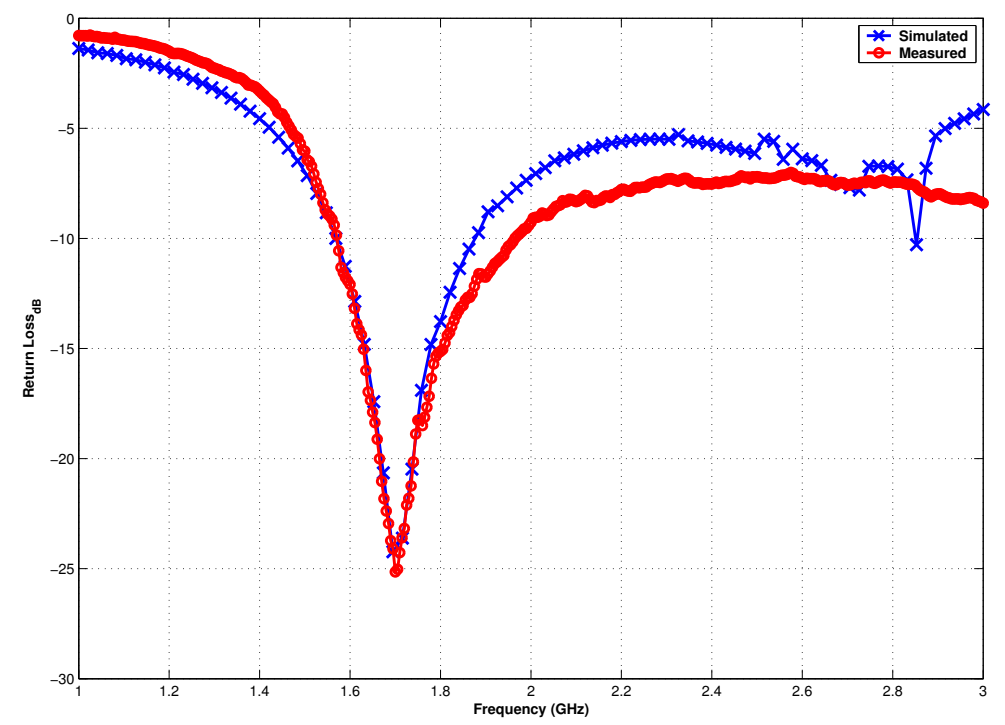

Figure 44.: Return loss.

\subsection{Comparison of Time/Memory Performance}

Having developed a semianalytical technique we expect a significatively improvements also in terms of time computation and memory requirement. In this section the performance of the IEM method in terms of computation time and memory requirement is investigated and compared with a popular commercial tool HFSS [54]. We have used a Matlab code to implement the IEM method. To perform the comparison we use to different structure: a three layer radome, a cylindrical reflector, Figure 46, and a circular conformal array of 8 conformal dipoles mounted around a cylindrical reflector, Figure 50ii. The characteristics of the radome and the cylindrical reflector are reported in Table 2 whereas the dipole dimensions and the array parameters are reported in Table 3 and Table 4 respectively. The dimension of the array's dipoles are identical to these indicated in Table 3. 


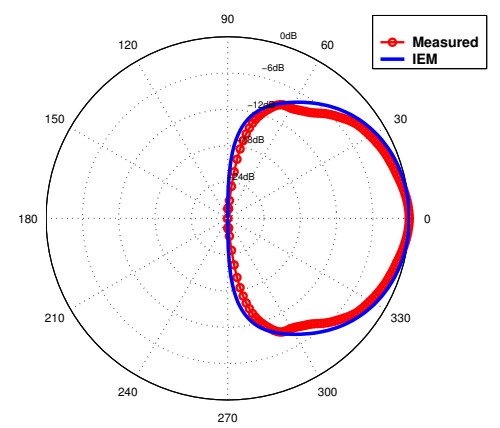

(i) VP simulated with IEM and Measured without radome.

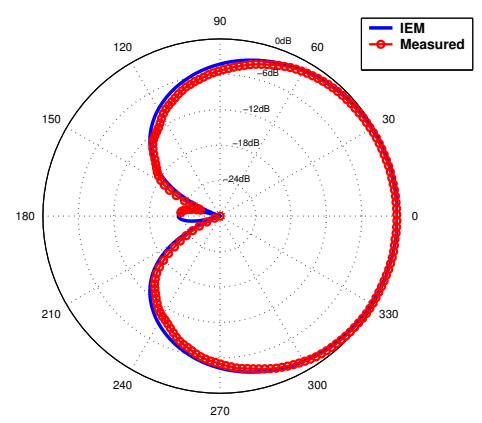

(iii) HP simulated with IEM and Measured without radome.

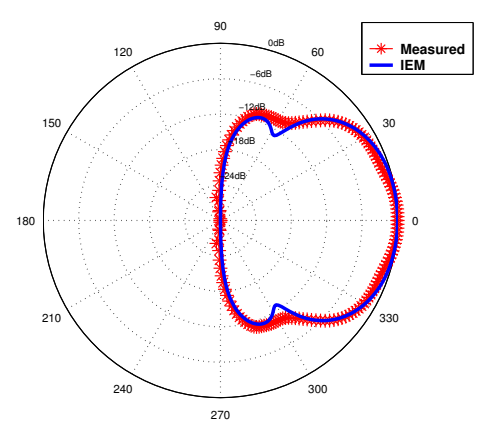

(ii) VP simulated with IEM and Measured with radome.

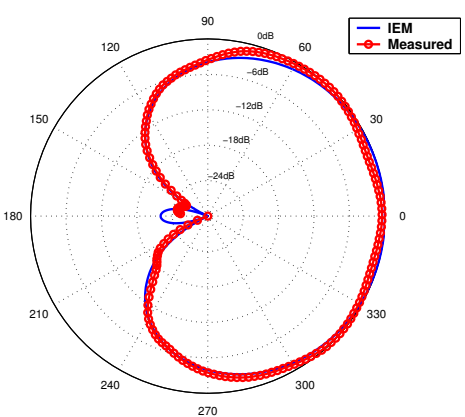

(iv) HP simulated with IEM and Measured with radome.

Figure 45.: Comparison between simulated with IEM and measured Horizontal(HP) and Vertical Pattern (VP) of the conformal finite length dipole at $1.75 \mathrm{GHz}$ at $3.88 \mathrm{~cm}$ from the cylindrical reflector. 
Table 2.: Simulated structure layout.

\begin{tabular}{lr}
\hline Cylindrical reflector diameter & $0.297 \mathrm{~m}$ \\
Diameter of the radome & $0.530 \mathrm{~m}$ \\
Layer 1 and 3 thicknesses & $0.004 \mathrm{~m}$ \\
Layer 2 & $0.021 \mathrm{~m}$ \\
Dielectric constant layer 1 and 3 & 4.5 \\
Dielectric constant layer 2 & 1.2
\end{tabular}

Table 3.: Dipole dimensions.

\begin{tabular}{lr}
\hline $\mathrm{L}$ & $0.092 \mathrm{~m}$ \\
$\mathrm{w}$ & $0.05 \mathrm{~m}$ \\
$\mathrm{~h}$ & $0.001 \mathrm{~m}$ \\
Location in $\rho$ & $\rho^{\prime}=0.1885 \mathrm{~m}$ \\
Layer 2 & $21 \mathrm{~m}$
\end{tabular}

The comparison is performed $2 \mathrm{GHz}$. The field computations results are compared in terms of far field horizontal pattern and good agreement can be observed between both methods, Figure 47 and Figure 50i.

\section{Performance with the radome and a conformal dipole.}

We perform the simulation in a desktop computer with processor $2.4 \mathrm{GHz}$ Intel Pentium 4 processor and 1 GB fast DDR RAM. Exploiting the symmetry properties of the cylindrical structure, with HFSS we can simulate $1 / 4$ of the structure. In this case approximately $33 \mathrm{k}$ tetrahedra [54] are needed for the simulation corresponding to $\sim 500 \mathrm{MB}$ memory allocation and $\backsim 40 \mathrm{~min}$ for the complete simulation. For the IEM based on Matlab code $\backsim 20 \mathrm{MB}$ are needed for the variables allocation and $\backsim 1.3$ min running time. 


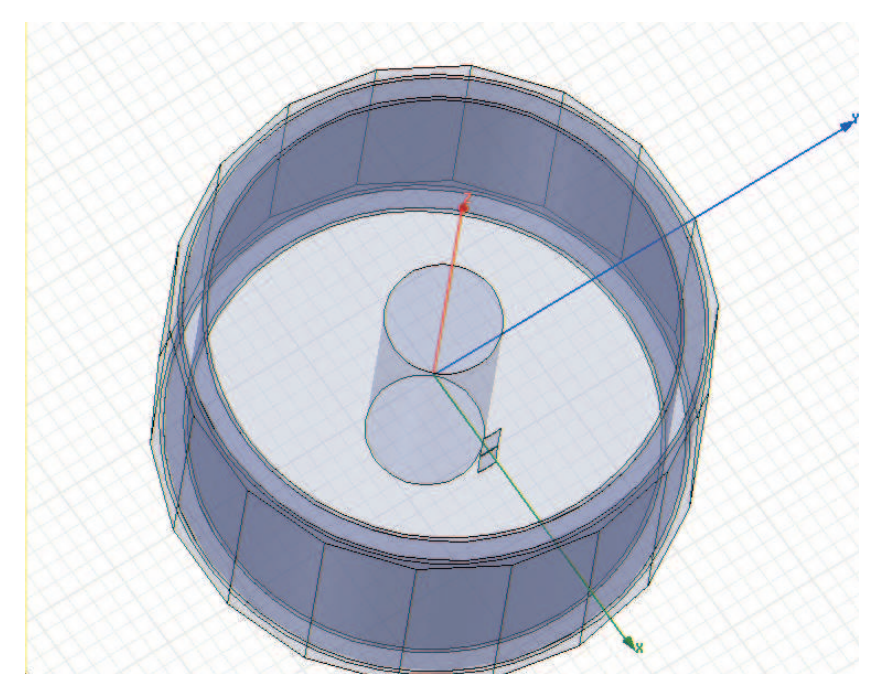

Figure 46.: Simulated structure.

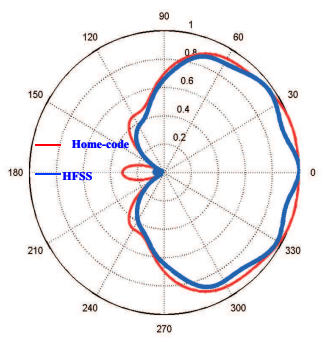

(i) Horizontal pattern computed with HFSS vs. IEM in linear scale.

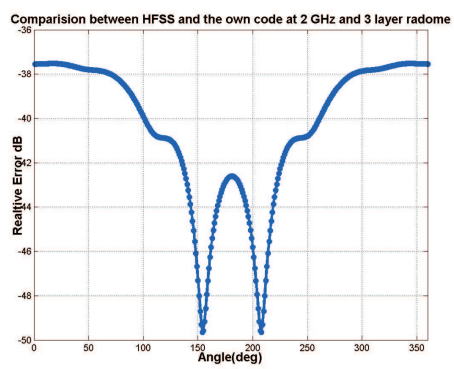

(ii) Relative error in $d B$ over $\varphi$.

Figure 47.: Comparison between the horizontal pattern computed with HFSS and IEM at $f=2 \mathrm{GHz}$. 
Table 4.: Array configuration dimensions.

\begin{tabular}{ll}
\hline Array diameter & $131 \mathrm{~mm}$ \\
Cylindrical reflector diameter & $123 \mathrm{~mm}$
\end{tabular}

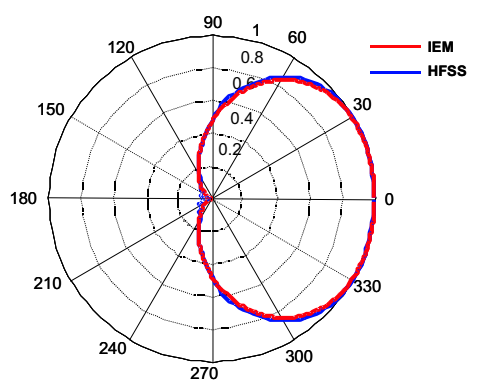

(i) Horizontal pattern computed with HFSS vs. IEM in linear scale.

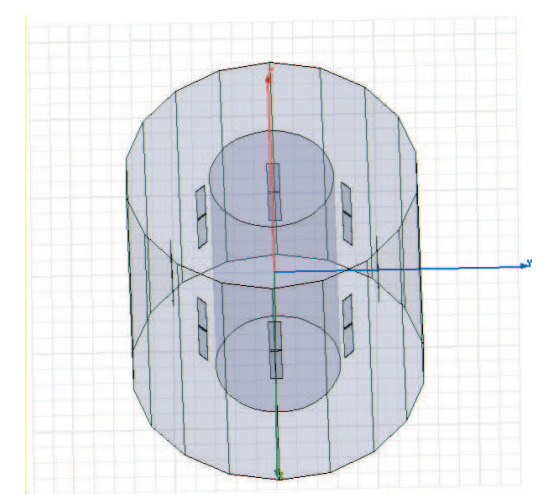

(ii) Array configuration with cylindrical reflector.

Figure 48.: Comparison between the horizontal pattern computed with HFSS and IEM at $f=2 \mathrm{GHz}$.

\section{Performance with array of conformal dipoles.}

Similarly to the previous case, we perform the simulation in a desktop computer with processor $2.4 \mathrm{GHz}$ Intel Pentium 4 processor and $1 \mathrm{~GB}$ fast DDR RAM. Exploiting the symmetry properties of the cylindrical structure, with HFSS we can simulate $1 / 4$ of the structure. In this case approximately $40 \mathrm{k}$ tetrahedra [54] are needed for the simulation corresponding to $\backsim 700 \mathrm{MB}$ memory allocation and $\backsim 70 \mathrm{~min}$ for the complete simulation. For the IEM based on Matlab code $\backsim 30 \mathrm{MB}$ are needed for the variables allocation and $\sim 1.5$ min running time. 


\subsection{Beamforming and Azimuth Scanning Application}

The use of circular antenna arrays for smart antenna application offers important and obvious advantages in smart antenna applications. Because the circular antenna array does not have edge elements, the directional patterns obtained by this geometry can be electronically scanned in the azimuth plane without significant changes of the beam shape. Recently it has been also proved that the uniform circular arrays present the best performance in terms of space resolution compared with uniform rectangular antenna array (URA) [44]. Even though they present the best performance in terms of space resolution, the most used antenna arrays for smart antenna application is the URA. This is due to the difficulties arising form the electromagnetic modelling in terms of computational resources as explained at the beginning of this work. Using the method developed in this work we show the possibility to investigate the performance of the smart antenna system presenting circular cylindrical symmetry combined on the fly with the electromagnetic simulation. To verify that we have implemented two simple algorithms. We consider a circular array of 8 conformal dipoles mounted around a cylindrical reflector with a without a 3 layers radome. The radome characteristics and the dimensions of the antenna array are reported in Table 2 and Table 4 respectively. In the first algorithm first a simple example of beamforming is presented. At the first step of the algorithm only one antenna element is excited. At the second step the neighbors elements placed at \pm 45 are excited with higher excitation voltages. At the third step the voltage values of the elements placed at \pm 45 is increased of a factor of 2. The simulation are performed with and without radome and the results for the azimuth plane are shown in Figure 49. While an evident effect of beamforming can be observed the simulation time with and without radome is approximately of $1,5 \mathrm{~min}$ and $3 \mathrm{~min}$ respectively. The second algorithm implements a beam scanning of the azimuth plane of 360 . The dipoles are excited progressively and one by one with a constant excitation voltage, Figure 50. In this case the simulation time for the cases with and without radome is $8 \mathrm{~min}$ and $12 \mathrm{~min}$ respectively. 


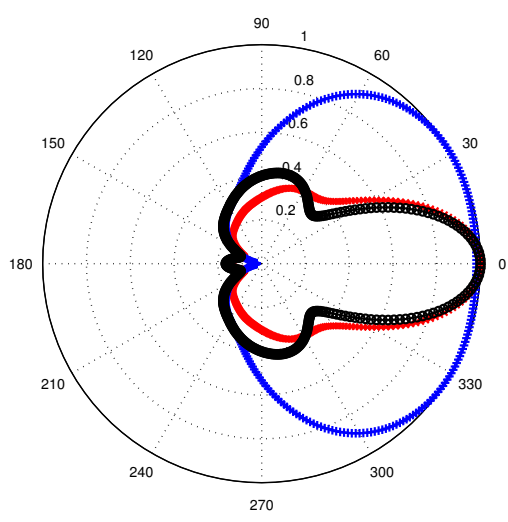

(i) Radiation pattern without radome.

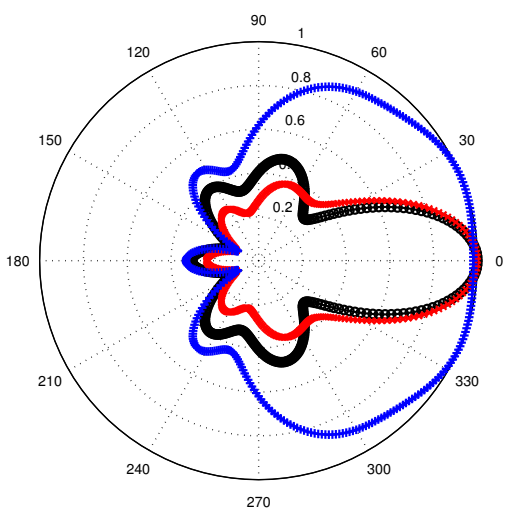

(ii) Radiation pattern with radome.

Figure 49.: In Figures an example of beamforing of the azimuth plane with and without radome is shown at $2 \mathrm{GHz}$.

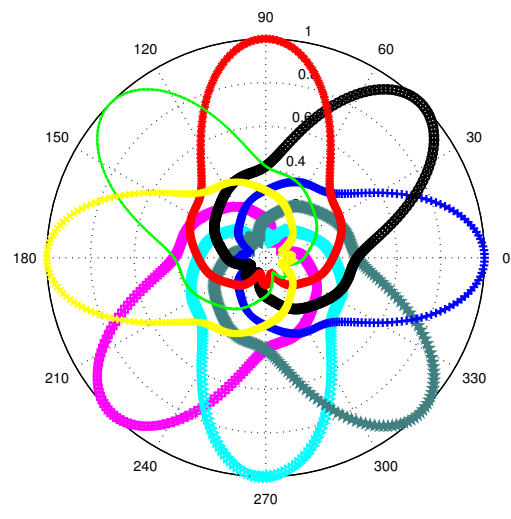

(i) Azimuth scanning without radome.

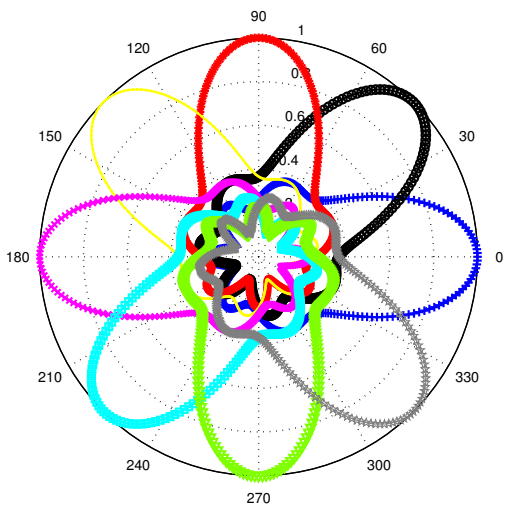

(ii) Azimuth scanning with radome.

Figure 50.: In Figures are shown simulations of electronic beam scanning with and without radome at $2 \mathrm{GHz}$. 



\section{Conclusions and Discussions}

We have presented theoretical methods, algorithms and implementations to deal with multilayered cylindrical radiating structures. The method is based on integral equation method (IEM). A novel method to compute the cylindrical multilayered dyadic Green's function has been introduced.

The method is based on a network description of the spectral domain (SD) cylindrical multilayered dyadic Green's function. The network is built using generalized transmission lines (GTL). We have shown that, due to the completeness of the transmission line theory, the GTL method introduces advantages in the implementation and design stage.

The convergence of the Green's function in the source region has been validated using two different reference models. The first reference model was derived considering that in the free space case the cylindrical Green's function can be easily expanded into spherical waves and a closed form solution can be obtained. However since the Green's function exhibits a singular behavior in the source region $\left(1 / \mathbf{r}^{3}\right)$, this simple model can be used as reference model only in region where the field exhibits a less singular behavior. This region can be estimated only numerically. In this case a good agreement with Green's function developed in this work is observed.

The problem of the singularity at the source region, from the theoretical point of view, can be described making use of the theory of distribution. In this case the field at the source can be computed analytically [41]. However following the theory of distribution, the computation of the field around the source involves a limit which is sensitive to numerical errors [41] and does not improve the accuracy of the reference model.

A more physical model to validate the convergence of the Green's function for small space variations around the source is introduced using, instead of an infinitesimal dipole, a small conformal radiation area. In this case for small region around the source, i.e., $\left|\mathbf{r}-\mathbf{r}^{\prime}\right|<\varepsilon$, the field can be approxi- 
mated to the static case. With this reference model we could achieve not divergent field for distances from the source of the order of $0.001 \lambda$ and test the Green's function convergence also in this region. Also in this case a good agreement with Green's function developed in this work was observed.

As mentioned, both reference models are derived in free space. More complex structures can be validated using other known Green's functions derived with other methods or experimental results. In our case we have applied the cylindrical Green's function with MOM to model two different structures: a conformal dipole antenna placed near a cylindrical reflector and embedded inside a cylindrical radome structure and a circular array of conformal dipoles mounted around a cylindrical reflector. Taking advantages of the rotation and translational invariant properties of the structures we have derived the method of moments (MOM) algorithm in spectral domain. While a good agreement with measurements and other commercial softwares was observed, an important improvement in terms of computation resources and understanding has been achieved. As further application, we have shown the potentialities of the method performing simple beamforming and azimuthal scanning algorithms performed quasi simultaneously with electromagnetic simulations. For this purpose, a circular array of 8 conformal dipoles was mounted around cylindrical reflector. The simulations where performed with and without a 3 layers cylindrical radome. The excellent performance in terms of computational time shows an attractive potentiality for full simulations of smart antenna systems based on multilayered cylindrical geometries.

\subsection{Outlook}

Further works related with the topic can be divided in different issues. The first issue concerns with the GPOF method. In this work the estimation of the number of the poles (model order), is done according to the singular values of the autocorrelation function of the data matrix. This is a good criterion for transient analysis in time domain [23]. In this case, the GPOF is applied to spatial signals for each mode $n$ and therefore to relatively smooth functions. This fact in some cases produces numerical instability. A better criteria to choose the model order should be introduced. 
The second issue concerns the further development of the GTL model. They can be used for application purposes to introduce a systematical beam shaping technique for cylindrical conformal antennas (e.g. ultra wide band applications) or design of cylindrical multilayered radomes. Some preliminary results have been presented in [14,8]. 



\section{A. Appendix}

\section{A.1. Potential Ansatz for $z$ and $\varphi$-oriented current}

In many practical cases, in stats of a more general exponential $\varphi$-dependency of the eigenfunctions $\mathrm{e}^{\left(j n\left(\varphi-\varphi^{\prime}\right)\right)}$, a sinusoidal dependency can be introduced. The field distribution depends also on the proprieties of the excitations. We consider a small dipole oriented in $z$-direction having a Dirac current distribution

$$
\underline{\mathcal{J}}=J_{0_{z^{\prime}}} \delta\left(\mathbf{r}-\mathbf{r}^{\prime}\right) \rho^{\prime} \mathrm{d} \rho^{\prime} \wedge \mathrm{d} \varphi^{\prime}
$$

In this case we can expect that the $E_{\rho}, E_{z}$ and $H_{\varphi}$ are symmetric in $\varphi$, while $E_{\varphi}, H_{\rho}$ and $H_{z}$ antisymmetric in $\varphi$. That must be reflected also in the magnetic vector potential form $\mathcal{A}$ which shows a cosine angular dependence while for the electric vector potential form $\mathcal{F}$ a sinusoidal angular dependence must be shown. Therefore excited modes by a small electrical dipole $z$-oriented can be represented by the following potential Ansatz:

$\left\{\begin{array}{l}\mathcal{A}^{i} \\ \mathcal{F}^{i}\end{array}\right\}=\mathrm{d} z \frac{j}{8 \pi} \sum_{n=0}^{+\infty} \int_{k_{z}}\left\{\begin{array}{l}\bar{\psi}_{\mathcal{A}}^{i}\left(\rho, \rho^{\prime} n, k_{z}\right) \\ \bar{\psi}_{\mathcal{F}}^{i}\left(\rho, \rho^{\prime} n, k_{z}\right)\end{array}\right\} \begin{aligned} & \cos \left(n\left(\varphi-\varphi^{\prime}\right)\right) \\ & \sin \left(n\left(\varphi-\varphi^{\prime}\right)\right)\end{aligned} e^{-j k_{z}\left(z-z^{\prime}\right)} d k_{z}$

Analogue consideration can be done for a small dipole $\varphi$-oriented having a Dirac current distribution

$$
\underline{\mathcal{J}}=J_{0_{\varphi^{\prime}}} \delta\left(\mathbf{r}-\mathbf{r}^{\prime}\right) \mathrm{d} z^{\prime} \wedge \mathrm{d} z^{\prime}
$$

In this case the Ansatz for the angular dependency, as excepted is specular

$$
\left\{\begin{array}{l}
\mathcal{A}^{i} \\
\mathcal{F}^{i}
\end{array}\right\}=\mathrm{d} z \frac{j}{8 \pi} \sum_{n=0}^{+\infty} \int_{k_{z}}\left\{\begin{array}{l}
\bar{\psi}_{\mathcal{A}}^{i}\left(\rho, \rho^{\prime} n, k_{z}\right) \\
\bar{\psi}_{\mathcal{F}}^{i}\left(\rho, \rho^{\prime} n, k_{z}\right)
\end{array}\right\} \begin{aligned}
& \sin \left(n\left(\varphi-\varphi^{\prime}\right)\right) \\
& \cos \left(n\left(\varphi-\varphi^{\prime}\right)\right)
\end{aligned} e^{-j k_{z}\left(z-z^{\prime}\right)} d k_{z}
$$





\section{B. Appendix}

The aim of this Appendix in to review shortly the basic of the complex parametric integration used in this work. A more detailed description is presented in many good mathematical text books as in $[25,34]$.

\section{B.1. Complex Path Integration}

We recall some useful properties of the complex parametric representation. We consider a function $f(z)=u(z)+i v(z)$ and a path $\Gamma$. We assume $f$ to be an analytic function in the complex $\Gamma$. We also assume, similarly to the Chapter 6 to give the path $\Gamma$ in its parametric representation. That means that we can write the complex variable $z$ as function of a parameter $t$ in the following way

$$
z(t)=x(t)+i y(t)
$$

In this case the integral of the function $f$ over the complex path $\Gamma$ can be written as follows

$$
\int_{\Gamma} f(z) d z=\int_{\Gamma}(u+i v)(d x+i d y)
$$

using the parametric description of the variable $z$ given in (217) we obtain

$$
\int_{\Gamma} f(z) d z=\int_{\Gamma} f(z(t)) \frac{d z}{d t} d t=\int_{t=0}^{1}(u(t)+i v(t))\left(\frac{d x}{d t}(t)+i \frac{d y}{d t}(t)\right) d t
$$


For a linear path along passing by two specified points of the complex plane $\left(x_{1}, y_{1}\right)$ and $\left(x_{2}, y_{2}\right)$ we have

$$
\begin{aligned}
x(t) & =x_{1}+\left(x_{2}-x_{1}\right) t \\
y(t) & =y_{1}+\left(y_{2}-y_{1}\right) t \\
\frac{d x}{d t} & =\left(x_{2}-x_{1}\right)=\Delta x \\
\frac{d y}{d t} & =\left(y_{2}-y_{1}\right)=\Delta y
\end{aligned}
$$

In this case (219) can be rewritten as follows

$$
\int_{\Gamma} f(z) d z=\int_{\Gamma} f(z(t)) \frac{d z}{d t} d t=(\Delta x+i \Delta y) \int_{t=0}^{1}(u(t)+i v(t)) d t
$$




\section{Appendix}

In Section 6.1.5 the numerical computation of the boundary condition has been introduced. The purpose of this Appendix is to introduce an algorithm to compute in most efficient way to solve the boundary conditions.

\section{C.1. A static recursive approach}

We may note that the matrix $\mathbf{L}$, introduced in Section 6.1.5 is a diagonal matrix. That is due to the fact that only adjacent layers interact. That suggest that each boundary surface can be treated separately. In the following, we seek, staring from (64) a new parametric representation of each boundary surface which may be used to reduce the problem. We consider the interface $M-1$ to $M$. From (64) the layer $M$ and $M-1$ is complectly described in parametric way as follows:

$$
\begin{gathered}
\left(\begin{array}{c}
\mathbf{F}_{M-1}^{+} \\
\mathbf{F}_{M-1}^{-}
\end{array}\right)=\left(\begin{array}{c}
\mathbf{M}^{M-1^{+}} \\
\mathbf{M}^{M-1^{-}}
\end{array}\right)_{<8 \times 4>}\left(\begin{array}{c}
A_{n}^{M-1} \\
B_{n}^{M-1} \\
C_{n}^{M-1} \\
D_{n}^{M-1}
\end{array}\right) \\
\left(\begin{array}{c}
\mathbf{F}_{M}^{+} \\
\mathbf{F}_{M}^{-}
\end{array}\right)=\left(\begin{array}{c}
\mathbf{M}^{M^{+}} \\
\mathbf{M}^{M^{-}}
\end{array}\right)_{<8 \times 4>}\left(\begin{array}{c}
B_{n}^{M} \\
D_{n}^{M}
\end{array}\right)
\end{gathered}
$$

By applying the boundary conditions between the layer $M-1$ and $M$, from the (90), we have that

$$
\mathbf{F}_{M-1}^{+}=\mathbf{F}_{M}^{-}
$$




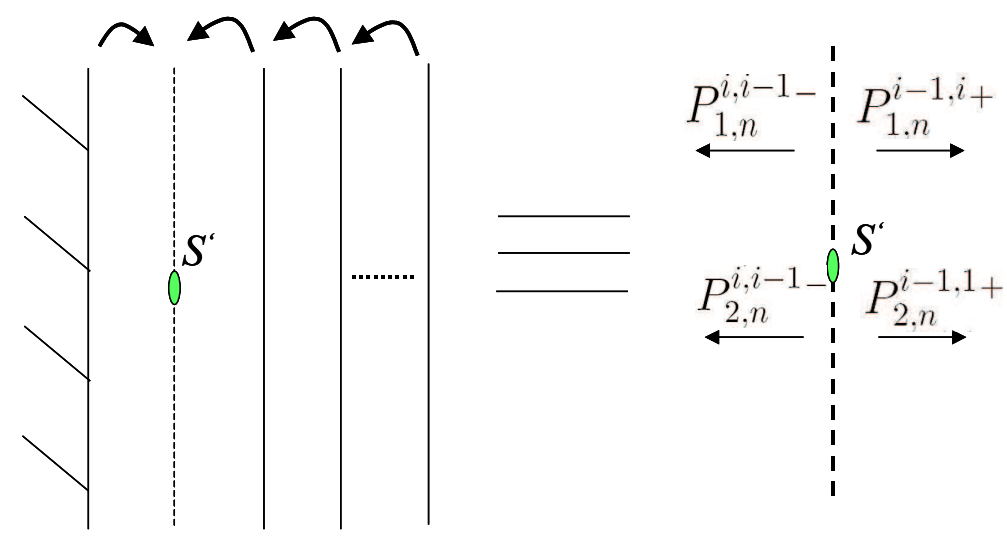

Figure 51.: In figure a qualitative description of the algorithm is given.

By using (226) and (227) we can rewrite (228) as following

$$
\left(\mathbf{M}^{M-1^{+}}-\mathbf{M}^{M-1^{-}}\right)_{<4 \times 6>}\left(\begin{array}{c}
A_{n}^{M-1} \\
B_{n}^{M-1} \\
C_{n}^{M-1} \\
D_{n}^{M-1} \\
B_{n}^{M} \\
D_{n}^{M}
\end{array}\right)=\left(\begin{array}{l}
0 \\
0 \\
0 \\
0
\end{array}\right)
$$

which is an under determined system of equations. Considering the non singular case, it exists a nullspace $\mathbf{K}$ of dimension two that salsifies (229). In other words, $\mathbf{K}$ can be seen as a base of two vectors $\mathbf{K}=\left(\mathbf{b}_{1}^{6 \times 1} \mathbf{b}_{2}^{6 \times 1}\right)$ that span the nullspace which is a solution of the (229)

$$
\left(\begin{array}{c}
A_{n}^{M-1} \\
B_{n}^{M-1} \\
C_{n}^{M-1} \\
D_{n}^{M-1} \\
B_{n}^{M} \\
D_{n}^{M}
\end{array}\right)=\left(\begin{array}{ll}
\mathbf{b}_{1} & \mathbf{b}_{2}
\end{array}\right)_{<6 \times 2>}\left(\begin{array}{l}
P_{1, n}^{M-1, M} \\
P_{2, n}^{M-1, M}
\end{array}\right)
$$




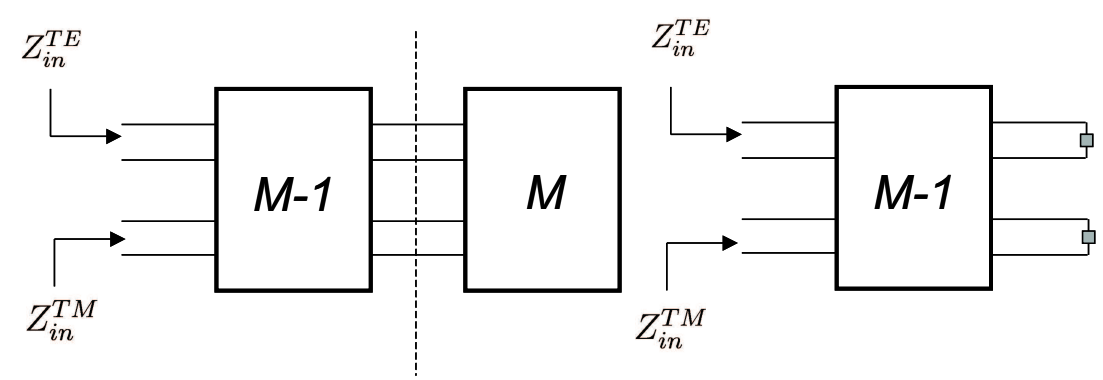

Figure 52.: In figure a qualitative description of the algorithm using the GTL model is given.

where $P_{l, n}^{i-1, i}$ are two unknown parameters and $1<i<M$. Analyzing the structure of the nullspace, we observe that the calculation of the fields at the interface $M-1$ are given by the first for lines of the nullspace $K_{<4 \times 2>}^{M-1}=$ $\left(\mathbf{b}_{1}^{4 \times 1} \mathbf{b}_{2}^{4 \times 1}\right)^{M-1}$ while the fields at the interface $M$ from the lower part $K_{<2 \times 2>}^{M}=\left(\mathbf{b}_{1}^{4 \times 1} \mathbf{b}_{2}^{2 \times 1}\right)^{M}$. Therefore, we can write that

$$
\begin{gathered}
\mathbf{F}^{M-1^{+}}=M_{4 \times 4}^{M-1^{+}}\left(\begin{array}{c}
A_{n}^{M-1} \\
B_{n}^{M-1} \\
C_{n}^{M-1} \\
D_{n}^{M-1}
\end{array}\right)=\left(\begin{array}{c}
P_{1, n}^{M-1, M} \\
P_{2, n}^{M-1, M}
\end{array}\right) \\
\mathbf{F}^{M^{-}}=M_{4 \times 4}^{M-1^{+}}\left(\begin{array}{c}
B_{n}^{M} \\
D_{n}^{M-1}
\end{array}\right)=M_{4 \times 4}^{M-1^{+}} K_{<4 \times 2>}^{M}\left(\begin{array}{c}
P_{1, n}^{M-1, M} \\
P_{2, n}^{M-1, M}
\end{array}\right)
\end{gathered}
$$

form (229) and using (230) we can rewrite the (231) as follows

$$
\mathbf{F}^{M^{-}}=M_{4 \times 2}^{M^{-}} K_{<2 \times 2>}^{M}\left(\begin{array}{c}
P_{1, n}^{M-1, M} \\
P_{2, n}^{M-1, M}
\end{array}\right)
$$

We have derived a new parametric description of the outer layer. An analogous formulation can be repeated starting from the innermost layer. The procedure can be recursively used starting form the innermost and outer most layer until the source interface is reached, Figure 51. In this way the 
overall structure has been reduced to the source interface which is described by 4 new parameters, 2 given by the right side recursion and 2 given by the left side recursion. Form the algebraic point of view we have a system of 4 unknown in 4 variables that can be easily solved. Using the GTL model the algorithm can be easily interpreted and implemented just reducing the outermost layer to its equivalent load recursively, Figure, until to obtain en equivalent network with the only current generator and equivalent loads. 


\section{Appendix}

In this Appendix some useful formulas for the antenna pattern of the conformal cylindrical antenna will be derived. To compute the far field pattern there are two basics approaches. The first one based on the equivalence principle, consists of taking the field over in a closed surface containing the sources. Then the field in the far field can be computed via applying a two dimensional Fourier transformation[citazione] In cases in which semianalytical techniques can be exploited, a more convenient approach is to use an asymptotic approximation for large distance from the antenna. In this case the antenna pattern can be better shaped due the knowledged of the Green's function $[14,8]$ as it is in this work.

\section{D.1. Field Computations}

The (46) and (47) report the field representation in terms of electric and magnetic and electric vector potential form $\mathcal{A}$ and $\mathcal{F}$. From the potential the electric and magnetic field components, for a circular cylindrical coordinates system can be computed as follows

$$
\begin{aligned}
E_{\rho} & =\frac{1}{j \omega \varepsilon} \frac{\partial^{2} \psi_{\mathcal{A}}}{\partial \rho \partial z} & H_{\rho} & =\frac{1}{\rho} \frac{\partial \psi_{\mathcal{A}}}{\partial \phi} \\
E_{\varphi} & =\frac{1}{j \omega \varepsilon \rho} \frac{\partial^{2} \psi_{a}}{\partial \phi \partial z} & H_{\varphi} & =-\frac{\partial \psi_{\mathcal{A}}}{\partial \rho} \\
E_{z} & =\frac{1}{j \omega \varepsilon}\left(\frac{\partial^{2}}{\partial z^{2}}+k^{2}\right) \psi_{\mathcal{A}} & H_{z} & =0
\end{aligned}
$$


and

$$
\begin{aligned}
E_{\rho} & =-\frac{1}{\rho} \frac{\partial \psi_{\mathcal{F}}}{\partial \phi} & H_{\rho} & =\frac{1}{j \omega \mu} \frac{\partial^{2} \psi_{\mathcal{F}}}{\partial \rho \partial z}, \\
E_{\varphi} & =\frac{\partial \psi_{\mathcal{F}}}{\partial \rho} & H_{\varphi} & =\frac{1}{j \omega \mu \rho} \frac{\partial^{2} \psi_{\mathcal{F}}}{\partial \phi \partial z}, \\
E_{z} & =0 & H_{z} & =\frac{1}{j \omega \mu}\left(\frac{\partial^{2}}{\partial z^{2}}+k^{2}\right) \psi_{\mathcal{F}}
\end{aligned}
$$

\section{D.1.1. Farfield Expression}

We consider a circular coordinates system as shown in Figure 53.

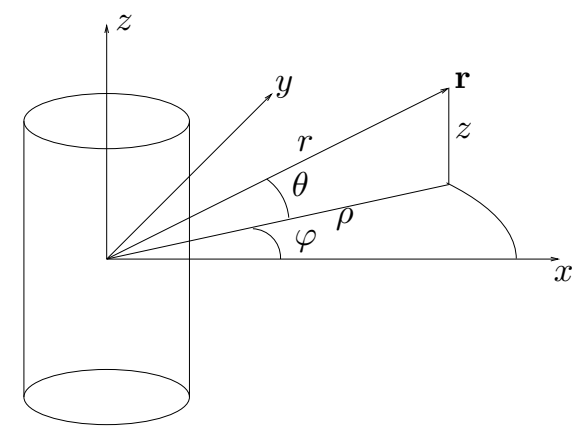

Figure 53.: Coordinate system for farfield calculations.

For convenience, we rewrite (60) inserting the axial density current dependency in SD

$$
\begin{aligned}
& \bar{\psi}_{\mathcal{F}}=\sum_{n=0}^{\infty} J\left(k_{z}\right)\left[A_{n} H_{n}^{(1)}\left(k_{\rho} \rho\right)+B_{n} H_{n}^{(2)}\left(k_{\rho} \rho\right)\right] \\
& \bar{\psi}_{\mathcal{A}}=\sum_{n=0}^{\infty} J\left(k_{z}\right)\left[C_{n} H_{n}^{(1)}\left(k_{\rho} \rho\right)+D_{n} H_{n}^{(2)}\left(k_{\rho} \rho\right)\right]
\end{aligned}
$$

To compute the radiation characteristic we consider of outermost layer $M$ to be the free space. We consider the antenna to be in the transmission mode (only outgoing cylindrical waves exist), and therefore $A_{n}^{M}=0 C_{n}^{M}=$ 
0. Moreover we assume to have an Hertzian dipole $\left(J\left(k_{z}\right)=1\right)$. In this case (240) becomes

$$
\begin{aligned}
& \bar{\psi}_{\mathcal{F}}=\sum_{n=0}^{\infty} B_{n}^{M} H_{n}^{(2)}\left(k_{\rho_{M}} \rho\right) \\
& \bar{\psi}_{\mathcal{A}}=\sum_{n=0}^{\infty} D_{n}^{M} H_{n}^{(2)}\left(k_{\rho_{M}} \rho\right)
\end{aligned}
$$

Also we consider the Hertzian dipole to an electrical dipole oriented in $z$-direction. Exploiting the symmetry property of the source introduced in the Appendix , the electric field form in the outermost layer can be written as follows

$\left.\mathcal{E}_{M}(\mathbf{r})=\sum_{n=0}^{\infty}\left\{\begin{array}{l}\cos \left(n\left(\varphi-\varphi^{\prime}\right)\right) \\ \sin \left(n\left(\varphi-\varphi^{\prime}\right)\right)\end{array}\right\} \int_{k_{z}} B_{n}^{M} H_{n}^{(2)}\left(k_{\rho_{M}} \rho\right)+D_{n}^{M} H_{n}^{(2)}\left(k_{\rho_{M}} \rho\right)\right) d k_{z}$

The kernel of the integral (242) contains Hankel functions of the second kind. Therefore to introduce an asymptotic approximation of the integral (242) we shall consider the following asymptotic behavior of the Hankel functions $[19,28]$

$$
\begin{array}{r}
H_{n}^{(2)}(x) \stackrel{x \rightarrow \infty}{\longrightarrow} \sqrt{\frac{2 j}{\pi x}} j^{n} e^{(-j x)} \\
\frac{d}{d x} H_{n}^{(2)}(x) \stackrel{x \rightarrow \infty}{\longrightarrow} \sqrt{\frac{2 j}{\pi x}} j^{n}(-j) e^{(-j x)}
\end{array}
$$

We use (243) to solve asymptotically the Fourier integral of the $E_{z}$ component. For the other field components only the results will be reported since the computation is lengthy and similar to the one for the $E_{z}$ component. 
Using (236) we compute the $E_{z}$ component of the electric field

$$
\begin{aligned}
E_{z} & =\frac{1}{j \omega \varepsilon}\left(\frac{\partial^{2}}{\partial z^{2}}+k^{2}\right) \sum_{n=0}^{\infty} \cos \left(n\left(\varphi-\varphi^{\prime}\right)\right) \int_{k_{z}} D_{n}^{M} H_{n}^{(2)}\left(k_{\rho} \rho\right) e^{j k_{z}\left(z-z^{\prime}\right)} \mathrm{d} k_{z} \\
& \left.=\frac{1}{j \omega \varepsilon} \sum_{n=0}^{\infty} \cos \left(n\left(\varphi-\varphi^{\prime}\right)\right) \int_{k_{z}} k_{\rho}^{2} D_{n}^{M} H_{n}^{(2)}\left(k_{\rho} \rho\right)\right) e^{j k_{z}\left(z-z^{\prime}\right)} \mathrm{d} k_{z}
\end{aligned}
$$

when $\rho \rightarrow+\infty$, using (243), (245) can be solved as follows

$$
\begin{array}{r}
\left.\frac{1}{j \omega \varepsilon} \sum_{n=0}^{\infty} \cos \left(n\left(\varphi-\varphi^{\prime}\right)\right) \int_{k_{z}=-\infty}^{+\infty} k_{\rho}^{2} D_{n}^{M} \sqrt{\frac{2 j}{\pi k_{\rho} \rho}} j^{n} e^{\left(-j k_{\rho} \rho\right.}\right) e^{j k_{z}\left(z-z^{\prime}\right)} \mathrm{d} k_{z} \\
=\frac{1}{j \omega \varepsilon} \sum_{n=0}^{\infty} \cos \left(n\left(\varphi-\varphi^{\prime}\right)\right) \int_{k_{z}=-\infty}^{+\infty} k_{\rho}^{2} D_{n}^{M} \sqrt{\frac{2 j}{\pi k_{\rho} \rho}} j^{n} e^{\left(j\left(k_{z} z-k_{\rho} \rho\right)\right)} \mathrm{d} k_{z} \\
=\frac{(1+j)}{j \omega \varepsilon} k_{\rho, 0}^{2} \frac{e^{-j k r}}{r} \sum_{n=0}^{\infty} j^{n} D_{n}^{M} \cos \left(n\left(\varphi-\varphi^{\prime}\right)\right)
\end{array}
$$

where we have used the relation [19]

$$
\int_{k_{z}=-k}^{+k} f\left(k_{z}\right) \sqrt{\frac{2 j}{\pi k_{\rho} \rho}} j^{n} e^{j\left(k_{z} z-k_{\rho} \rho\right)} \mathrm{d} k_{z} \stackrel{\rho \rightarrow \infty}{\longrightarrow} f\left(k_{z, 0}\right) \frac{1}{r} j^{n}(1+j) e^{(-j k r)}
$$

which is valid for

$$
\begin{aligned}
k_{z} & =-k \sin \theta \\
k_{\rho} & =k \cos \theta \\
k^{2} & =k_{z}^{2}+k_{\rho}^{2}=k_{z}^{2}+k_{\rho}^{2} \\
r & =\rho \sqrt{1+\tan ^{2} \theta} \\
z & =\rho \tan \theta
\end{aligned}
$$

From (246) it is understood that for a fixed observation point, the phase with which modes of different $k_{\rho}$ contribute to the field varies very fast. 
That is due to the exponential dependency $e^{\left(-j k_{\rho} \rho\right)}$ where for large values $\rho$ varies fast with $k_{\rho}$. On the other hands, the modal amplitudes $D_{n}^{M}$, are slowly varying terms. That means that modes with modes are cancelled out for rapid phase changes and do not contribute to the integra. The only modes that can contribute in the far field are the are with stationary phase $\left(d / d k_{z}\left(k_{z} z-k_{\rho} \rho\right)=0\right)$. In this case a contribution to the integral occurs. In other words, only cylindrical modes with $k_{z}=-k \sin \theta$ generate a farfield at the elevation angle $\theta$. The azimuthal pattern is determined by a weighted sum of all the angular modes.

\section{D.1.2. Farfield expression for cylindrical and spherical coordinate}

In this section the expression of for the far field field component in cylindrical and spherical coordinates will be given.

We star with the farfield expressions for TM waves will be given

$$
\begin{aligned}
& E_{\rho}=\frac{(1+j)}{j \omega \varepsilon} k_{\rho, 0} k_{z, 0} \frac{e^{-j k r}}{r} \sum_{n=0}^{\infty} j^{n} D_{n}^{M} \cos \left(n\left(\varphi-\varphi^{\prime}\right)\right) \\
& E_{\phi}=0 \\
& E_{z}=\frac{(1+j)}{j \omega \varepsilon} k_{\rho, 0}^{2} \frac{e^{-j k r}}{r} \sum_{n=0}^{\infty} j^{n} D_{n}^{M} \cos \left(n\left(\varphi-\varphi^{\prime}\right)\right) \\
& H_{\rho}=0 \\
& H_{\phi}=(1+j)(j) k_{\rho, 0} \frac{e^{-j k r}}{r} \sum_{n=0}^{\infty} j^{n} D_{n}^{M} \cos \left(n\left(\varphi-\varphi^{\prime}\right)\right) \\
& H_{z}=0
\end{aligned}
$$

Since we are in far field, to obtain the field in spherical coordinates, it makes 
sense to transform the coordinate and obtain

$$
\begin{aligned}
& E_{r}=0 \\
& E_{\theta}=\frac{(1+j)}{j \omega \varepsilon} k^{2} \frac{e^{-j k r}}{r} \cos \theta \sum_{n=0}^{\infty} j^{n} D_{n}^{M} \cos \left(n\left(\varphi-\varphi^{\prime}\right)\right) \\
& E_{\phi}=0, \\
& H_{r}=0, \\
& H_{\theta}=0, \\
& H_{\phi}=(1+j)(j) k \frac{e^{-j k r}}{r} \cos \theta \sum_{n=0}^{\infty} j^{n} D_{n}^{M} \cos \left(n\left(\varphi-\varphi^{\prime}\right)\right)
\end{aligned}
$$

For transversal electric modes, the same calculations can be repeated and only expression in spherical coordinates are reported

$$
\begin{aligned}
& E_{r}=0, \\
& E_{\theta}=0, \\
& E_{\varphi}=-(1+j)(j) k \frac{e^{-j k r}}{r} \cos \theta \sum_{n=0}^{\infty} j^{n} B_{n}^{M} \sin \left(n\left(\varphi-\varphi^{\prime}\right)\right. \\
& H_{r}=0, \\
& H_{\theta}=\frac{(1+j)}{j \omega \mu} k^{2} \frac{e^{-j k r}}{r} \cos \theta \sum_{n=0}^{\infty} j^{n} B_{n}^{M} \sin \left(n\left(\varphi-\varphi^{\prime}\right)\right. \\
& H_{\varphi}=0
\end{aligned}
$$

Analyzing the fields one can find out, that TM modes produce a farfield with only an $E_{\theta}$ and an $H_{\varphi}$ component. Similarly, TE modes only have an $E_{\varphi}$ and an $H_{\theta}$ component. I both cases electric and magnetic field are orthogonal and related by the impedance of the outermost medium (i.e. typically the free-space impedance). The Poynting vector is directed outward, as expected. Fields generated by $T M$ and TE modes are orthogonal. 


\section{E. Appendix}

\section{E.1. Exterior Differential forms}

Scalar and vector fields may be represented by exterior differential forms, which are essentially the expression under an integration symbol. In order to describe this differential notation, it is shown in Table 5 along with the corresponding vector notation for some of the most relevant electromagnetic laws and relations.

A complete and detailed treatment of the differential forms can be found in $[21,29,55]$.

In a three dimensional curvilinear coordinate system with coordinates $\boldsymbol{\rho}=(u$, $v, w)$, there are four differential forms given by

$$
\begin{aligned}
\text { zero-form (scalar): } & \varphi(\boldsymbol{r}) \\
\text { one-form (vector) : } & \mathcal{E}(\boldsymbol{r})=E_{u} s_{1}+E_{v} s_{2}+E_{w} s_{3} \\
\text { two-form (pseudovector) : } & \mathcal{D}(\boldsymbol{r})=D_{u} s_{2} \wedge s_{3}+D_{v} s_{3} \wedge s_{1}(271) \\
& +D_{w} s_{1} \wedge s_{2} \\
\text { three-form (pseudoscalar) : } & \mathcal{Q}(\boldsymbol{r})=Q s_{1} \wedge s_{2} \wedge s_{3}
\end{aligned}
$$

whereas $s_{1}, s_{2}$ and $s_{3}$ are referred as the unit forms and have been already defined in Chapter 5 . 
Table 5.: Correspondences between the main electromagnetic equations in conventional vector notation and exterior differential form notation in the Laplace domain

\begin{tabular}{|c|c|c|}
\hline Equation & Vector Notation & Differential Notation \\
\hline Ampère's & & \\
\hline law & $\operatorname{curl} \boldsymbol{H}=s \boldsymbol{D}+\boldsymbol{J}$ & $d \mathcal{H}=s \mathcal{D}+\mathcal{J}$ \\
\hline Faraday's & & \\
\hline law & $\operatorname{curl} \boldsymbol{E}=-s \boldsymbol{B}$ & $d \mathcal{E}=-s \mathcal{B}$ \\
\hline law & $\operatorname{div} \boldsymbol{D}=\rho$ & $d \mathcal{D}=\mathcal{Q}$ \\
\hline $\begin{array}{l}\text { Magnetic flux } \\
\text { continuity }\end{array}$ & $\operatorname{div} \boldsymbol{B}=0$ & $d \mathcal{B}=0$ \\
\hline $\begin{array}{l}\text { Electric constitutive } \\
\text { equation }\end{array}$ & $\boldsymbol{D}=\varepsilon \boldsymbol{E}$ & $\mathcal{D}=\star \varepsilon \mathcal{E}$ \\
\hline $\begin{array}{l}\text { Magnetic constitutive } \\
\text { equation }\end{array}$ & $\boldsymbol{B}=\mu \boldsymbol{H}$ & $\mathcal{B}=\star \mu \mathcal{H}$ \\
\hline $\begin{array}{l}\text { Electric boundary } \\
\text { condition }\end{array}$ & $\boldsymbol{n} \times\left(\boldsymbol{E}_{2}-\boldsymbol{E}_{1}\right)=-\boldsymbol{J}_{m A}$ & \lrcorner$\wedge\left(\mathcal{E}_{2}-\mathcal{E}_{1}\right)=-\mathcal{J}_{m A}$ \\
\hline $\begin{array}{l}\text { Magnetic boundary } \\
\text { condition } \\
\text { Electric flux }\end{array}$ & $\boldsymbol{n} \times\left(\boldsymbol{H}_{2}-\boldsymbol{H}_{1}\right)=\boldsymbol{J}_{e A}$ & \lrcorner$\wedge\left(\mathcal{H}_{2}-\mathcal{H}_{1}\right)=\mathcal{J}_{e A}$ \\
\hline $\begin{array}{l}\text { boundary condition } \\
\text { Magnetic flux }\end{array}$ & $\boldsymbol{n} \cdot\left(\boldsymbol{D}_{2}-\boldsymbol{D}_{1}\right)=\rho_{s}$ & \lrcorner$\wedge\left(\mathcal{D}_{2}-\mathcal{D}_{1}\right)=\mathcal{Q}_{A}$ \\
\hline $\begin{array}{l}\text { boundary condition } \\
\text { Source power }\end{array}$ & $\boldsymbol{n} \cdot\left(\boldsymbol{B}_{2}-\boldsymbol{B}_{1}\right)=0$ & \lrcorner$\wedge\left(\mathcal{B}_{2}-\mathcal{B}_{1}\right)=0$ \\
\hline density & $d P_{s}=-\frac{1}{2}\left(\boldsymbol{E} \cdot \boldsymbol{J}_{e A}^{*}+\boldsymbol{E} \cdot \boldsymbol{J}_{e A}^{*}\right)$ & 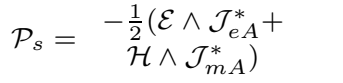 \\
\hline $\begin{array}{l}\text { Power loss } \\
\text { density } \\
\omega \mu^{\prime \prime} \overline{2 \mathcal{H} \wedge \mathcal{H}^{*}} \\
\text { Electric energy }\end{array}$ & $d P_{L}=\frac{\omega \varepsilon^{\prime \prime}}{2} \boldsymbol{E} \cdot \boldsymbol{E}^{*}+\frac{\omega \mu^{\prime \prime}}{2} \boldsymbol{H} \cdot \boldsymbol{H}^{*}$ & $\mathcal{P}_{L}=\frac{\omega \mathcal{E}^{\prime \prime}}{2} \mathcal{E} \wedge \star \mathcal{E}^{*}+$ \\
\hline $\begin{array}{l}\text { density } \\
\text { Magnetic energy }\end{array}$ & $d W_{e}=\frac{\varepsilon}{4} \boldsymbol{E} \cdot \boldsymbol{E}^{*}$ & $\mathcal{W}_{e}=\frac{\varepsilon}{4} \mathcal{E} \wedge \mathcal{E}^{*}$ \\
\hline $\begin{array}{l}\text { density } \\
\text { Complex Poynting's }\end{array}$ & $d W_{m}=\frac{\mu}{4} \boldsymbol{H} \cdot \boldsymbol{H}^{*}$ & $\mathcal{W}_{m}=\frac{\mu}{4} \mathcal{H} \wedge \mathcal{H}^{*}$ \\
\hline $\begin{array}{l}\text { vector } \\
\text { Complex Poynting's }\end{array}$ & $\boldsymbol{T}=\frac{1}{2} \boldsymbol{E} \times \boldsymbol{H}^{*}$ & $\mathcal{T}=\frac{1}{2} \mathcal{E} \wedge \mathcal{H}^{*}$ \\
\hline theorem & $\begin{array}{c}\boldsymbol{T} \cdot \boldsymbol{n} d S=-2 s\left(d W_{m}-d W_{e}\right) \\
-d P_{L}+d P_{0}\end{array}$ & $\begin{array}{c}d \mathcal{T}=-2 s\left(\mathcal{W}_{m}-\mathcal{W}_{e}\right) \\
-\mathcal{P}_{L}+\mathcal{P}_{0}\end{array}$ \\
\hline
\end{tabular}




\section{Bibliography}

[1] Z. Xiang and Y. Lu, "Electromagnetic Dyadic Green's Function in Cylindrically Multilayered Media", IEEE Trans. on Microwave Theory and Techniques, vol. 44, no. 4, April 1996.

[2] C. T. Tai, "Dyadic Green's Function for Cylindrical Waveguide with Moving Medium", Appli. Sci., vol. 25, pp. 281-289, 1971.

[3] C. T. Tai, "Dyadic Green's function for a coaxial line.", IEEE Trans. Antenna Propagat., vol. 31, pp. 355-358, 1983.

[4] W. C. Chew, "Waves and fields in inhomogeneous media.", New York: MacGraw-Hill, 1985.

[5] W. Yin and W. Wang, "Green's Function of Cylindrical Multilayered Chiral Media and its Application.", J. Electromag. Wave Applicat., vol. 7, pp. 1005-1027, 1993.

[6] Ç. Tokgöz and G. Düral, "Closed-form Green's functions for Cylindrical Stratified Media", IEEE Trans. on Microwave Theory and Techniques, vol. 48, no. 1, January 2000.

[7] Chen-To Tai, "Dyadic Green Functions in Electromagnetic Theory", 2nd. ed. New York: IEEE, 1993.

[8] B. Biscontini, M. Burger, F. Demmel, P. Russer, " An accurate fastalgorithm for analyzing and optimizing cylindrically stratified radomes", Microwaves, Radar and Wireless Communications, 2004. MIKON-2004. 15th International Conference on Volume 1, 17-19 May 2004 Page(s):259 - 263 Vol.1. 
[9] P. Lorenz, J. V. Vital, B. Biscontini and P. Russer, "High-Throughput Transmission Line Matrix (TLM) System in Grid Environment for Microwave Design, Analysis and Optimizations", IMS, Long Beach, Califormia, 2005.

[10] P. Lorenz, J. V. Vital, B. Biscontini and P. Russer, "High-Throughput Transmission Line Matrix (TLM) System in Grid Environment for the Analysis of Complex Electromagnetic Structures", ACES, 2005.

[11] P. Lorenz, J. V. Vital, B. Biscontini and P. Russer, "TLM-G: A Gridenabled Time Domain Transmission Line Matrix System for the Analysis of Complex Electromagnetic Structures", MTT IEEE Trans., 2005, to be publisched in December 2005.

[12] B. Biscontini, M. Burger, F. Demmel, P. Russer, "Dyadic Green's function for conformal antennas in multi layered cylindrical structures using generalized transmission lines", Microwave Conference, 2004. 34th European Volume 2, 13 Oct. 2004 Page(s):953 - 956.

[13] B. Biscontini, M. Burger and P. Russer, "Fields, Networks, Computational Methods, and Systems in Modern Electromagnetics, A tribute to Leopold B. Felsen", Springer Proceedings in Physics, Berlin, Heidelberg Springer, vol. 97, pp. 129-142, 2004.

[14] B. Biscontini and P. Russer, "A Design Approach for Radiating Cylindrical Multilayered Structures.", Microwave Conference, 2005. Accepted paper.

[15] Yingbo Hua nad T. Sakar, "Generalized-Pencil-of-Function Method for extracting Poles for an EM System form Its Transient Responce", IEEE Trans. on Antennas and Propagation, vol. 37, no. 2, Febbruary 1989.

[16] M.I. Aksum, "A Robust approach for the derivation of closed form Green's functions", IEEE Trans. on Antennas and Propagation, vol. 44, pp. 651-658, May 1996. Y. Hua and T. Sarkar, Matrix pencil

[17] Y. Hua and T. Sarkar," Matrix pencil method for estimating parameters of exponentially damped/undamped sinusoids in noise", IEEE Trans. Acoust., Speech, Signal Processing 38 (1990), no. 5, 814-824.

[18] J. Sun, C.F. Wang, L.W. Li , M.S. Leong, "Closed-form Green's functions for $\rho$-Oriented Electrical Source in Cylindrical Stratified Media", 
Antennas and Propagation Society International Symposium, 2002. IEEE , Volume: 2, 16-21 June 2002.

[19] R. F. Harrington, "Time-Harmonic Electromagnetic Fields", New York: McGrawHill, 1961.

[20] R. F. Harrington, "Fields computation by method of moment", New York: The Macmillan company, 1968.

[21] Peter Russer, Electromagnetics, microwave circuits and antenna design for communication engineering, Arthec house inc., 2003.

[22] V.B. Yurchenko, A. Altintaş, A.I. Nosich, "Numerical Optimization of a Cylindrical Reflector-in-Radome Antenna System", New York: McGrawHill, 1961.

[23] Fabio Coccetti, "Application of System Identification (SI) to Full-Wave Time Domain Characterization of Microwave and Millimeter Wave Passive Structures", PhD. Thesis, München, 2004.

[24] "MATLAB user manual".

[25] Erwin Kreyszig, "Advanced Engineering Mathematic ", Jhon Wiley Sons, Inc., Seventh Edition, 1993.

[26] Weng Cho Chew, "Singularities of the Fourier-Type Integral in a Multicylindrical Layer Problems", IEEE Transactions on Antennas and Propagation,vol. AP-31, July 1983.

[27] Akira Ishimaru, "Electromagnetic Wave Propagation, Radiation and Scattering", Englewood Cliffs, New Jersey: Prentice-Hall, 1991.

[28] Constantine A. Balanis, "Antenna theory analysis and design", John Wiley and Sons,Inc, Newe York, 1997.

[29] K.F. Warnick and D. Arnold, "Electromagnetic Green's function using differential forms", J.Electromagn. Waves and Appl.,vol. 10, no. 3, pp. 427-438, 1996.

[30] G. de Rham, "Differential Manifolds", J.Electromagn. Waves and Appl., New York: Springer, 1984.

[31] R.E. Collin, "Field theory of guided waves", IEEE Press, Inc., New York, 1991. 
[32] K. Naihadham and L.B. Felsen, "Dispersion of Waves Guided Along a Cylindrical Substrate-Superstrate Layered Medium ", IEEE Trans in Ante.,, Inc., New York, 1991.

[33] T. Rozzi and M. Mogiardo," open electromagnetic waveguides", IEE Electromagnetic wave series, UK, 1997.

[34] L.-S. Hahn and B. Epstein, "Classical Compelx Analysis", Joanes and Barlett, London, 1996.

[35] Y.L. Chow, J.J. Yang, D.G. Fang and G.E. Howard, " A Closed-Form Spatial Green's Function for the Thick Microstrip Substrate", IEEE Trans. Microwave Theory techn., vol. 39, no. 3, pp. 588-592, March 1991.

[36] R. Prony,"Essai experimental et analytique sur les lois de la dilatabilit'e de fluides elastiques et sur celles del la force expansive de la vapeur de l'alkool, 'a differentes temperatures", J. l'Ecole Polytech., Paris, 1795.

[37] V.K. Jain, T.K Sarkar and D.D. Weiner, "Rational Modeling by Pencilof-Function Method", IEEE Trans. Acoust., Speech, Signal Processing, vol. ASSP-31, no. 3, pp. 564-573, June 1983.

[38] G.H. Golub and C.F. Van Loan, "Matrix Computations.", Baltimore", MD: Johns Hopkins Univ., 1983.

[39] A. D Yaghjian, "Electric Dyadic Green's Functions in the Source Region",Proc. IEEE, vol. 68, No. 2,February 1980.

[40] A. D Yaghjian, " A Unified and Consistent View on the Singularities of the Electric Dyadic Green's Function in the Source region",IEEE Trans. On Antenna and Prop., vol. AP-20, No. 3,May 1982.

[41] J. Van Bladel, "Singular Electromagnetic Fields and Sources ",IEEE Press.,Inc., 1995.

[42] J.A. Stratton, "Electromagnetic Theory ", Mc-Graw Hill, NY, 1941.

[43] P. Ioannides and C. A. Balanis, " Uniform Circular Arrays for Smart Antennas ", Antennas and Propagation Society International Symposium, IEEE Vol. 3, pp. 2796-2799, June 2004.

[44] P. Ioannides and C. A. Balanis, " Uniform Circular Array and Rectangular array for Adaptive Beamforming Applications ", IEEE Antennas and Wireless Propagation Letters, Vol. 4, 2005. 
[45] D. G. Fang, J. J. Yang and G. Y Delisle, " Discrete Image Theory Horizontal Electric Dipole in a Multilayer Medium.", Proc. Inst. Elect. Eng., pt. H, vol. 135, pp. 297-303, Oct. 1988.

[46] J.J. Yang, Y.L. Chow, G.E. Howard and D.G. Fang, " Complex Image of an Electric Dipole in a Homogeneous and Layered Dielectric Between Two Grounded Planes.", IEEE Trans. Microwave Theory techn., vol. 40, pp. 595-600, March 1992.

[47] J. Sun, C. F. Wang,L. W. Li and M. S. Leong, " Further Improvements for Fast Computation of Mixed Potential Green's Functions for Cylindrical Stratified Media", IEEE Trans. on Atennas and Propagat., vol. 52,no. 11, pp. 3026-3036, March 2004.

[48] J. Sun, C. F. Wang,L. W. Li and M. S. Leong, " A Complete Set of Spatial Domain Dyadic Green's Functions for Components for Cylindrically Stratified Media in Fest Computational Form.", J. Electromagn. Waves Appl., vol. 16,no. 11, pp. 1491-1509, 2002.

[49] R. C. Hall, C. H. Thng and D. C. Chang , "Further Improvements for Fast Computation of Mixed Potential Green's Functions for Cylindrical Stratified Media", IEEE Trans. on Atennas and Propagat., vol. 52,no. 11, pp. 3026-3036, March 2004.

[50] M. Abramowitz and I. A. Stegun, "Handbook of Mathematical Functions with Formulas, Graphs, and Mathematical Tables", Dover Publications, 1972.

[51] James Clerk Maxwell, "A Treatise on Electricity and Magnetism", Clarendon Press (Oxford), Dover edition, New York, 1954.

[52] S. Ramo, J. Whiennery and T. Van Durez, "Fields and waves in communication electronics,", Third edition, Jhon Wiley Sons, Inc., 1994.

[53] K.F. Warnik, R. H. Selfridge, D. V. Arnold, " Teaching electromagnetic field theory using differential forms,", Education, IEEE Transactions on Volume 40, Issue 1, Feb. 1997 Page(s):53 - 68.

[54] HFSS, User manual .

[55] Alain Bossavit, "Differential Geometry for the student of numerical methods in Electromagnetism", 
http://www.lgep.supelec.fr/mse/perso/ab/DGSNME.pdf, August 1991.

[56] WinFEKO, User manual . 